\title{
EXTENSIONS OF LINKING SYSTEMS WITH $p$-GROUP KERNEL
}

\author{
BOB OLIVER AND JOANA VENTURA
}

\begin{abstract}
We study extensions of $p$-local finite groups where the kernel is a $p$ group. In particular, we construct examples of saturated fusion systems $\mathcal{F}$ which do not come from finite groups, but which have normal $p$-subgroups $A \triangleleft \mathcal{F}$ such that $\mathcal{F} / A$ is the fusion system of a finite group. One of the tools used to do this is the concept of a "transporter system", which is modelled on the transporter category of a finite group, and is more general than a linking system.
\end{abstract}

Let $G$ be a finite group, with Sylow $p$-subgroup $S \in \operatorname{Syl}_{p}(G)$. The fusion system of $G$ (at $p$ ) is the category $\mathcal{F}_{S}(G)$ whose objects are the subgroups of $G$, and where $\operatorname{Mor}_{\mathcal{F}_{S}(G)}(P, Q)$ is the set of monomorphisms from $P$ to $Q$ induced by conjugation by elements of $G$. The transporter system of $G$ at $p$ is the category $\mathcal{T}_{S}(G)$ with the same objects as $\mathcal{F}_{S}(G)$, and with morphism sets $\operatorname{Mor}_{\mathcal{T}_{S}(G)}(P, Q)=N_{G}(P, Q)$ : the set of elements of $G$ which conjugate $P$ into $Q$. A subgroup $P \leq S$ is called $p$-centric in $G$ if $C_{G}(P)=Z(P) \times C_{G}^{\prime}(P)$ for a (unique) subgroup $C_{G}^{\prime}(P)$ of order prime to $p$; and the centric linking system of $G$ is the category $\mathcal{L}_{S}^{c}(G)$ whose objects are the subgroups of $S$ which are $p$-centric in $G$, and where $\operatorname{Mor}_{\mathcal{L}_{S}^{c}(G)}(P, Q)=N_{G}(P, Q) / C_{G}^{\prime}(P)$. All of these definitions are repeated in more detail at the beginning of Section 1. In several papers, such as [BLO1] and [O2], the fusion and linking systems of $G$ are shown to play a central role in describing homotopy theoretic properties of the $p$-completed classifying space $B G_{p}^{\wedge}$.

Abstract fusion and linking systems have also been defined and studied, and are shown in [BLO2] to have many of the same properties as the fusion and linking systems of finite groups. A p-local finite group is defined to be a triple $(S, \mathcal{F}, \mathcal{L})$, where $S$ is a finite $p$-group, $\mathcal{F}$ is a saturated fusion system over $S$ (Definitions 1.2 and 1.3), and $\mathcal{L}$ is a centric linking system associated to $\mathcal{F}$ (Definition 1.6). Normal and central $p$-subgroups of fusion systems and linking systems are also defined (Definition 1.4).

Certain types of extensions of $p$-local finite groups, and in particular central extensions, were studied in [BCGLO2]. One hope was that extensions could provide a new way to construct exotic examples. But in the case of central extensions, this was shown to be impossible. By [BCGLO2, Theorem 6.13 and Corollary 6.14], if $A$ is a central subgroup in $(S, \mathcal{F}, \mathcal{L})$, and $(S / A, \mathcal{F} / A, \mathcal{L} / A)$ is induced by a group $G$, then $(S, \mathcal{F}, \mathcal{L})$ is induced by a group $\widetilde{G}$ such that $A \leq Z(\widetilde{G})$ and $\widetilde{G} / A \cong G$.

In this paper, we look at the more general situation of extensions with $p$-group kernel. Equivalently, given a $p$-local finite group $(S, \mathcal{F}, \mathcal{L})$ and a finite $p$-group $A$, we want to find $p$-local finite groups $(\widetilde{S}, \widetilde{\mathcal{F}}, \widetilde{\mathcal{L}})$ such that $A \triangleleft \mathcal{F}$ and $(S, \mathcal{F}, \mathcal{L}) \cong$ $(\widetilde{S} / A, \widetilde{\mathcal{F}} / A, \widetilde{\mathcal{L}} / A)$. One problem when doing this is that the fusion system $\mathcal{F} / A$ contains too little information: $\mathcal{F}$ cannot be described as an extension of $\mathcal{F} / A$ by $A$ in any sense.

2000 Mathematics Subject Classification. Primary 55R35. Secondary 55R40, 20D20.

Key words and phrases. Classifying space, $p$-completion, finite groups, fusion.

B. Oliver is partially supported by UMR 7539 of the CNRS.

J. Ventura is partially supported by FCT/POCTI/FEDER and grant PDCT/MAT/58497/2004.

Both authors were partially supported by the Mittag-Leffler Institute in Sweden. 
Another problem is that in general, when $\widetilde{\mathcal{L}}$ is a linking system and $A \triangleleft \widetilde{\mathcal{L}}$, then $\widetilde{\mathcal{L}}$ can be thought of as an extension of $\widetilde{\mathcal{L}} / A$ by $A$, but $\widetilde{\mathcal{L}} / A$ need not be a linking system. As explained in Section 2, it can contain much more information than a linking system does. Conversely, if we take a linking system $\mathcal{L}$ and try naively to extend it, then the resulting category will in general have too few objects to be a linking system. So we were forced to look at a larger class of categories to extend.

A transporter system is a category whose objects are subgroups of a given $p$-group $S$, associated to a given fusion system, which satisfies axioms motivated by the two main examples: the transporter category of a finite group, and categories of the form $\mathcal{L} / A$ when $\mathcal{L}$ is a linking system and $A \triangleleft \mathcal{L}$. A transporter system $\mathcal{T}$ for which $\operatorname{Ob}(\mathcal{T})$ is the set of all subgroups of $S$ is always the transporter category of the finite group $\operatorname{Aut}_{\mathcal{T}}(1)$ (Proposition 3.12), so we are interested mainly in the cases where not all subgroups of $S$ are objects. The precise definition is given at the start of Section 3.

Nerves of transporter systems have many of the topological properties which are already known for linking systems. For example, if $\mathcal{T}$ is a transporter system associated to the fusion system $\mathcal{F}$, and $\operatorname{Ob}(\mathcal{T})$ includes all $\mathcal{F}$-centric subgroups, then $\mathcal{T}$ induces a centric linking system $\mathcal{L}$ associated to $\mathcal{F}$ and $|\mathcal{T}|_{p}^{\wedge} \simeq|\mathcal{L}|_{p}^{\wedge}$ (Proposition 4.6). As another example, if $\mathcal{T}^{r} \subseteq \mathcal{T}$ is the full subcategory whose objects are the " $\mathcal{T}$-radical subgroups" (Definition 3.9), then $\left|\mathcal{T}^{r}\right| \simeq|\mathcal{T}|$ (Proposition 4.7).

Extensions of transporter systems are defined and studied in Section 5. If $\mathcal{T}$ is a transporter system, and $\tau: \widetilde{\mathcal{T}} \rightarrow \mathcal{T}$ is a functor which satisfies certain category theoretic properties (Definition 5.1), then $\widetilde{\mathcal{T}}$ is also a transporter system and $\widetilde{\mathcal{T}} / A \cong \mathcal{T}$ for a certain normal $p$-subgroup $A \triangleleft \widetilde{\mathcal{T}}$. Moreover, in this situation, $|\widetilde{\mathcal{T}}| \rightarrow|\mathcal{T}|$ is a fibration with fiber $B A$. Once this has been established, then conditions are described (Theorem 5.11) which imply that $\widetilde{\mathcal{T}}$ is in fact a centric linking system, or at least a full subcategory of a centric linking system which includes all subgroups which are centric and radical.

Finally, in Section 6 , we look at extensions $1 \rightarrow A \rightarrow \widetilde{\mathcal{T}} \rightarrow \mathcal{T} \rightarrow 1$ of this type, when $\mathcal{T}$ is a full subcategory of the transporter category of a finite group $G$. We first show that if the induced action of $\pi_{1}(|\mathcal{T}|)$ on $A$ factors through $G$, then $\tilde{\mathcal{T}}$ will be a full subcategory of the transporter category of some group $\widetilde{G}$ such that $\widetilde{G} / A \cong G$. Afterwards, we give examples (Example 6.2) of such extensions where the action does not factor through $G$, and where $\widetilde{\mathcal{T}}$ and its associated fusion system $\widetilde{\mathcal{F}}$ are exotic in in the sense that $\widetilde{\mathcal{F}}$ is not the fusion system of any finite group.

We would like to thank the University of Aberdeen, the Universitat Autònoma de Barcelona, and especially the Bernoulli Center in Lausanne and the Mittag-Leffler Institut near Stockholm for their hospitality, allowing the two authors to meet together and work on this project. We would also like to thank Albert Ruiz for his very timely discovery of some examples of exotic fusion systems which helped lead to our Example 6.2 .

\section{BACKGROUND: FUSION AND LINKING SYSTEMS}

We first fix some notation. For any group $G$, and any $x \in G, c_{x}$ denotes conjugation by $x\left(c_{x}(g)=x g x^{-1}\right)$. For $H, K \leq G$, we write

$$
N_{G}(H, K)=\left\{x \in G \mid x H x^{-1} \leq K\right\}
$$


to denote the transporter set, and

$$
\operatorname{Hom}_{G}(H, K)=\left\{c_{x} \in \operatorname{Hom}(H, K) \mid x \in N_{G}(H, K)\right\} \cong N_{G}(H, K) / C_{G}(H) .
$$

We also set $\operatorname{Aut}_{G}(H)=\operatorname{Hom}_{G}(H, H) \cong N_{G}(H) / C_{G}(H)$.

Definition 1.1. Fix a finite group $G$ and a Sylow subgroup $S \in \operatorname{Syl}_{p}(G)$.

(a) $\mathcal{F}_{S}(G)$ and $\mathcal{T}_{S}(G)$ denote the categories where $\operatorname{Ob}\left(\mathcal{F}_{S}(G)\right)=\mathrm{Ob}\left(\mathcal{T}_{S}(G)\right)$ is the set of all subgroups of $S$, and where

$$
\operatorname{Mor}_{\mathcal{F}_{S}(G)}(P, Q)=\operatorname{Hom}_{G}(P, Q) \cong N_{G}(P, Q) / C_{G}(P)
$$

and

$$
\operatorname{Mor}_{\mathcal{T}_{S}(G)}(P, Q)=N_{G}(P, Q) .
$$

Let $\rho: \mathcal{T}_{S}(G) \longrightarrow \mathcal{F}_{S}(G)$ be the functor which is the identity on objects, and which sends $x \in N_{G}(P, Q)$ to $c_{x} \in \operatorname{Hom}_{G}(P, Q)$.

(b) A p-subgroup $P \leq G$ is p-centric in $G$ if $Z(P) \in \operatorname{Syl}_{p}\left(C_{G}(P)\right)$; equivalently, if $C_{G}(P)=Z(P) \times C_{G}^{\prime}(P)$ for some (unique) subgroup $C_{G}^{\prime}(P)$ of order prime to $p$. Define $\mathcal{L}_{S}^{c}(G)$ to be the category whose objects are the subgroups of $S$ which are $p$-centric in $G$, and where

$$
\operatorname{Mor}_{\mathcal{L}_{S}^{c}(G)}(P, Q)=N_{G}(P, Q) / C_{G}^{\prime}(P) .
$$

We call $\mathcal{F}_{S}(G)$ the fusion system (or fusion category) of $G, \mathcal{T}_{S}(G)$ the transporter system, and $\mathcal{L}_{S}^{c}(G)$ the centric linking system. In this paper, we will be looking at abstract versions of all three of these systems, starting with fusion systems.

Definition 1.2 ([Pg], [BLO2, Definition 1.1]). A fusion system over a finite p-group $S$ is a category $\mathcal{F}$, where $\operatorname{Ob}(\mathcal{F})$ is the set of all subgroups of $S$, and which satisfies the following two properties for all $P, Q \leq S$ :

- $\operatorname{Hom}_{S}(P, Q) \subseteq \operatorname{Hom}_{\mathcal{F}}(P, Q) \subseteq \operatorname{Inj}(P, Q)$; and

- each $\varphi \in \operatorname{Hom}_{\mathcal{F}}(P, Q)$ is the composite of an isomorphism in $\mathcal{F}$ followed by an inclusion.

Fusion systems as defined above are too general for our purposes, and additional axioms are needed for them to be very useful. When $\mathcal{F}$ is a fusion system over a finite $p$-subgroup $S$, then two subgroups $P, Q \leq S$ are said to be $\mathcal{F}$-conjugate if they are isomorphic as objects of the category $\mathcal{F}$. A subgroup $P \leq S$ is called fully centralized in $\mathcal{F}$ if $\left|C_{S}(P)\right| \geq\left|C_{S}\left(P^{\prime}\right)\right|$ for all $P^{\prime} \leq S$ which is $\mathcal{F}$-conjugate to $P$. Similarly, a subgroup $P \leq S$ is called fully normalized in $\mathcal{F}$ if $\left|N_{S}(P)\right| \geq\left|N_{S}\left(P^{\prime}\right)\right|$ for all $P^{\prime} \leq S$ which is $\mathcal{F}$-conjugate to $P$.

Definition 1.3 ([Pg], [BLO2, Definition 1.2]). A fusion system $\mathcal{F}$ over a finite p-group $S$ is saturated if the following two conditions hold:

(I) For all $P \leq S$ which is fully normalized in $\mathcal{F}, P$ is fully centralized in $\mathcal{F}$ and $\operatorname{Aut}_{S}(P) \in \operatorname{Syl}_{p}\left(\operatorname{Aut}_{\mathcal{F}}(P)\right)$.

(II) If $P \leq S$ and $\varphi \in \operatorname{Hom}_{\mathcal{F}}(P, S)$ are such that $\varphi P$ is fully centralized, and if we set

$$
N_{\varphi}=\left\{g \in N_{S}(P) \mid \varphi c_{g} \varphi^{-1} \in \operatorname{Aut}_{S}(\varphi P)\right\},
$$

then there is $\bar{\varphi} \in \operatorname{Hom}_{\mathcal{F}}\left(N_{\varphi}, S\right)$ such that $\left.\bar{\varphi}\right|_{P}=\varphi$. 
If $G$ is a finite group and $S \in \operatorname{Syl}_{p}(G)$, then $\mathcal{F}_{S}(G)$ is a saturated fusion system. Axioms (I) and (II) follow mostly as consequences of the Sylow theorems (cf. [BLO2, Proposition 1.3]).

We next specify certain collections of subgroups relative to a given fusion system.

Definition 1.4. Let $\mathcal{F}$ be a fusion system over a finite $p$-subgroup $S$.

- A subgroup $P \leq S$ is $\mathcal{F}$-centric if $C_{S}\left(P^{\prime}\right)=Z\left(P^{\prime}\right)$ for all $P^{\prime} \leq S$ which is $\mathcal{F}$ conjugate to $P$. We let $\mathcal{F}^{c} \subseteq \mathcal{F}$ denote the full subcategory with objects the $\mathcal{F}$-centric subgroups of $S$.

- A subgroup $P \leq S$ is $\mathcal{F}$-radical if $\operatorname{Out}_{\mathcal{F}}(P)$ is p-reduced; i.e., if $O_{p}\left(\operatorname{Out}_{\mathcal{F}}(P)\right)=1$.

- A subgroup $A \leq S$ is normal in $\mathcal{F}$ (denoted $A \triangleleft \mathcal{F}$ ) if for all $P, Q \leq S$ and all $f \in \operatorname{Hom}_{\mathcal{F}}(P, Q), f$ extends to a morphism $\bar{f} \in \operatorname{Hom}_{\mathcal{F}}(P A, Q A)$ such that $\bar{f}(A)=A$.

- A subgroup $A \leq S$ is central in $\mathcal{F}$ if for all $P, Q \leq S$ and all $f \in \operatorname{Hom}_{\mathcal{F}}(P, Q), f$ extends to a morphism $\bar{f} \in \operatorname{Hom}_{\mathcal{F}}(P A, Q A)$ such that $\left.\bar{f}\right|_{A}=\operatorname{Id}_{A}$.

If $\mathcal{F}=\mathcal{F}_{S}(G)$ for some finite group $G$, then $P \leq S$ is $\mathcal{F}$-centric if and only if $P$ is $p$-centric in $G$ (i.e., $Z(P) \in \operatorname{Syl}_{p}\left(C_{G}(P)\right)$ ), and $P$ is $\mathcal{F}$-radical if and only if $N_{G}(P) /\left(P \cdot C_{G}(P)\right)$ is $p$-reduced. However, $P$ being $\mathcal{F}$-radical is not the same as being a radical $p$-subgroup.

In fact, it turns out that saturated fusion systems defined only on the centric subgroups are equivalent to saturated fusion systems defined on all subgroups. In other words, when constructing saturated fusion systems over a finite $p$-group $S$, we really need only define it on the centric subgroups of $S$, and check that it satisfies axioms (I) and (II) for those subgroups. The next theorem describes how a category constructed in this way can then be extended in a unique way to a saturated fusion system over $S$.

For any fusion system $\mathcal{F}$ over $S$, and any set $\mathcal{H}$ of subgroups of $S$ which is closed under $\mathcal{F}$-conjugacy, we say that $\mathcal{F}$ is $\mathcal{H}$-saturated if conditions (I) and (II) in Definition 1.3 are satisfied for all $P \in \mathcal{H}$. We say that $\mathcal{F}$ is $\mathcal{H}$-generated if each morphism in $\mathcal{F}$ is a composite of restrictions of morphisms between subgroups in $\mathcal{H}$.

Theorem 1.5. Fix a p-group $S$ and a fusion system $\mathcal{F}$ over $S$.

(a) Assume $\mathcal{F}$ is saturated, and let $\mathcal{H}$ be the set of $\mathcal{F}$-centric $\mathcal{F}$-radical subgroups of $S$. Then $\mathcal{F}$ is $\mathcal{H}$-generated. More precisely, for each $P, P^{\prime} \leq S$ and each $\varphi \in \operatorname{Iso}_{\mathcal{F}}\left(P, P^{\prime}\right)$, there are subgroups $P=P_{0}, P_{1}, \ldots, P_{k}=P^{\prime}$, subgroups $Q_{i} \geq$ $\left\langle P_{i-1}, P_{i}\right\rangle(i=1, \ldots, k)$ which are $\mathcal{F}$-centric, $\mathcal{F}$-radical, and fully normalized in $\mathcal{F}$, and automorphisms $\varphi_{i} \in \operatorname{Aut}_{\mathcal{F}}\left(Q_{i}\right)$, such that $\varphi_{i}\left(P_{i-1}\right)=P_{i}$ for all $i$ and $\varphi=\left(\left.\varphi_{k}\right|_{P_{k-1}}\right) \circ \cdots \circ\left(\left.\varphi_{1}\right|_{P_{0}}\right)$.

(b) Let $\mathcal{F}$ be a fusion system over a finite p-group $S$. Let $\mathcal{H}$ be a set of subgroups of $S$ closed under $\mathcal{F}$-conjugacy such that $\mathcal{F}$ is $\mathcal{H}$-saturated and $\mathcal{H}$-generated. Assume also that each $\mathcal{F}$-centric subgroup of $S$ not in $\mathcal{H}$ is $\mathcal{F}$-conjugate to some subgroup $P \leq S$ such that $\operatorname{Out}_{S}(P) \cap O_{p}\left(\operatorname{Out}_{\mathcal{F}}(P)\right) \neq 1$. Then $\mathcal{F}$ is saturated.

Proof. Part (a) is Alperin's fusion theorem for saturated fusion systems, in the form shown in [BLO2, Theorem A.10]. Part (b) is proven in [BCGLO1, Theorem 2.2].

We now turn to linking systems associated to abstract fusion systems.

Definition 1.6 ([BLO2, Definition 1.7]). Let $\mathcal{F}$ be a fusion system over the p-group $S$. A centric linking system associated to $\mathcal{F}$ is a category $\mathcal{L}$ whose objects are the 
$\mathcal{F}$-centric subgroups of $S$, together with a functor $\pi: \mathcal{L} \longrightarrow \mathcal{F}^{c}$, and distinguished monomorphisms $P \stackrel{\delta_{P}}{\longrightarrow} \operatorname{Aut}_{\mathcal{L}}(P)$ for each $\mathcal{F}$-centric subgroup $P \leq S$, which satisfy the following conditions.

(A) $\pi$ is the identity on objects. For each pair of objects $P, Q \in \mathcal{L}, Z(P)$ acts freely on $\operatorname{Mor}_{\mathcal{L}}(P, Q)$ by composition (upon identifying $Z(P)$ with $\delta_{P}(Z(P)) \leq \operatorname{Aut}_{\mathcal{L}}(P)$ ), and $\pi$ induces a bijection

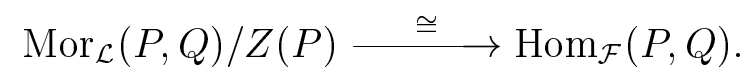

(B) For each $\mathcal{F}$-centric subgroup $P \leq S$ and each $x \in P, \pi\left(\delta_{P}(x)\right)=c_{x} \in \operatorname{Aut}_{\mathcal{F}}(P)$.

(C) For each $f \in \operatorname{Mor}_{\mathcal{L}}(P, Q)$ and each $x \in P$, the following square commutes in $\mathcal{L}$ :

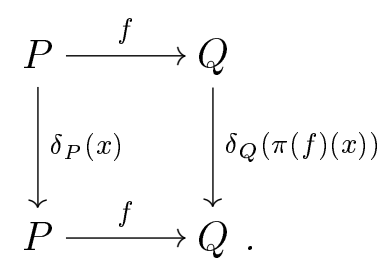

A $p$-local finite group is defined to be a triple $(S, \mathcal{F}, \mathcal{L})$, where $S$ is a finite $p$-group, $\mathcal{F}$ is a saturated fusion system over $S$, and $\mathcal{L}$ is a centric linking system associated to $\mathcal{F}$. The classifying space of the triple $(S, \mathcal{F}, \mathcal{L})$ is the $p$-completed nerve $|\mathcal{L}|_{p}^{\wedge}$.

For any finite group $G$ with Sylow $p$-subgroup $S$, the category $\mathcal{L}_{S}^{c}(G)$ (Definition 1.1) is easily seen to satisfy conditions (A), (B), and (C) above, and hence is a centric linking system associated to $\mathcal{F}_{S}(G)$. Thus $\left(S, \mathcal{F}_{S}(G), \mathcal{L}_{S}^{c}(G)\right)$ is a $p$-local finite group, with classifying space $\left|\mathcal{L}_{S}^{c}(G)\right|_{p}^{\wedge} \simeq B G_{p}^{\wedge}$ (see [BLO1, Proposition 1.1]).

The following lifting lemma for linking systems is used frequently.

Lemma 1.7. Let $(S, \mathcal{F}, \mathcal{L})$ be a p-local finite group. Fix $\mathcal{F}$-centric subgroups $P, Q, R$ in $S$, and let $P \stackrel{\varphi}{\longrightarrow} Q \stackrel{\psi}{\longrightarrow} R$ be any sequence of morphisms in $\mathcal{F}$. Then the following hold.

(a) Let $\widetilde{\psi}$ and $\widetilde{\psi} \varphi$ be arbitrary liftings to $\mathcal{L}$ of $\psi$ and $\psi \varphi$, respectively. Then there is a unique morphism $\widetilde{\varphi} \in \operatorname{Mor}_{\mathcal{L}}(P, Q)$ such that

$$
\widetilde{\psi} \circ \widetilde{\varphi}=\widetilde{\psi \varphi}
$$

and furthermore $\pi_{P, Q}(\widetilde{\varphi})=\varphi$.

(b) Choose liftings $\widetilde{\psi \varphi} \in \operatorname{Mor}_{\mathcal{L}}(P, R)$ and $\widetilde{\varphi} \in \operatorname{Mor}_{\mathcal{L}}(P, Q)$ of $\psi \varphi$ and $\varphi$, respectively. Then there is a unique morphism $\widetilde{\psi} \in \operatorname{Mor}_{\mathcal{L}}(Q, R)$ such that $\widetilde{\psi} \circ \widetilde{\varphi}=\widetilde{\psi \varphi}$, and such that $\pi(\widetilde{\psi})=\psi \circ c_{q}$ for some $q \in \varphi(Z(P))$.

Proof. (a) See [BLO2, Lemma 1.10] or Lemma A.7(a).

(b) Let $\alpha \in \operatorname{Mor}_{\mathcal{L}}(Q, R)$ be a lifting of $\psi$, i.e., $\pi(\alpha)=\psi$. Then by axiom (A) of a linking system, there is $z \in Z(P)$ such that $\widetilde{\psi \varphi}=\alpha \circ \widetilde{\varphi} \circ \delta_{P}(z)$, and by axiom (C) we have $\widetilde{\varphi} \circ \delta_{P}(z)=\delta_{Q}(\varphi(z)) \circ \widetilde{\varphi}$. Set $\widetilde{\psi}=\alpha \circ \delta_{Q}(\varphi(z))$, and note that $\pi(\widetilde{\psi})=\psi \circ c_{\varphi(z)}$.

If $\widetilde{\psi^{\prime}}$ is another morphism satisfying the same conditions, then by assumption, $\pi\left(\widetilde{\psi}^{\prime}\right)=\pi(\widetilde{\psi}) \circ c_{x}$ for some $x \in \varphi(Z(P))$. Then by axiom $(\mathrm{A}), \widetilde{\psi}^{\prime}=\widetilde{\psi} \circ \delta_{Q}(\varphi(y))$ for some $y \in Z(P)$ such that $\varphi(y) \in x \cdot Z(Q)$. Also,

$$
\widetilde{\psi} \circ \widetilde{\varphi}=\widetilde{\psi \varphi}=\widetilde{\psi}^{\prime} \circ \widetilde{\varphi}=\widetilde{\psi} \circ \delta_{Q}(\varphi(y)) \circ \widetilde{\varphi}=\widetilde{\psi} \circ \widetilde{\varphi} \circ \delta_{P}(y)
$$


where the last equality follows from (C). Since the action of $Z(P)$ on $\operatorname{Mor}_{\mathcal{L}}(P, R)$ is free, it follows that $y=1$, and hence that $\widetilde{\psi}^{\prime}=\widetilde{\psi}$.

The following is an easy corollary to Lemma 1.7(a).

Corollary 1.8. Let $\mathcal{F}$ be a fusion system (not necessarily saturated) over a p-group $S$, and let $\mathcal{L}$ be a centric linking system associated to $\mathcal{F}$. For each $\mathcal{F}$-centric subgroup $P \leq S$, choose an "inclusion" morphism $\iota_{P} \in \operatorname{Mor}_{\mathcal{L}}(P, S)$ such that $\pi\left(\iota_{P}\right)=\operatorname{incl}_{P, S}$. Then there are unique injections $\delta_{P, Q}: N_{G}(P, Q) \longrightarrow \operatorname{Mor}_{\mathcal{L}}(P, Q)$, for each pair of subgroups $P, Q \in \mathrm{Ob}(\mathcal{L})$, with the property that $\iota_{Q} \circ \delta_{P, Q}(g)=\delta_{S}(g) \circ \iota_{P}$ for all $g \in$ $N_{G}(P, Q)$. Also, $\delta_{P}$ is the restriction to $P \leq N_{S}(P)$ of $\delta_{P, P}$ for each $P$, and the $\delta_{P, Q}$ define a functor from the transporter category of $S$ (restricted to the objects of $\mathcal{L}$ ) to $\mathcal{L}$.

Proof. See [BLO2, Proposition 1.11].

We finish the section by noting the following standard result in group theory which will be needed later.

Lemma 1.9. (a) If $Q \triangleleft P$ are finite p-groups and $\alpha \in \operatorname{Aut}(P)$ is such that $\left.\alpha\right|_{Q}=\operatorname{Id}_{Q}$ and $\alpha / Q=\operatorname{Id}_{P / Q}$, then $\alpha$ has $p$-power order.

(b) If $S$ is a p-subgroup of the finite group $G$, and $H \triangleleft G$, then $S \in \operatorname{Syl}_{p}(G)$ if and only if $S \cap H \in \operatorname{Syl}_{p}(H)$ and $S H / H \in \operatorname{Syl}_{p}(G / H)$.

Proof. Point (a) is shown in [Go, Corollary 5.3.3]. Point (b) follows since $S /(S \cap H) \cong$ $S H / H$ and hence

$$
[G: S]=[H: S \cap H] \cdot[G / H: S H / H]
$$

\section{QUOTIENTS OF LINKING SYSTEMS}

In this section, we show that whenever $(S, \mathcal{F}, \mathcal{L})$ is a $p$-local finite group and $A \triangleleft \mathcal{F}$ (Definition 1.4), then we can define a quotient $p$-local finite group $\left(S / A, \mathcal{F} / A,(\mathcal{L} / A)_{*}^{c}\right)$ as a quotient of $(S, \mathcal{F}, \mathcal{L})$ by $A$. We also show that $\mathcal{L} / A$, defined as the quotient of the free action of $A$ on $\mathcal{L}$, is not in general a linking system; and this will motivate the concept of a transporter system defined in the next section.

We first consider quotients of fusion systems. Recall that for any fusion system $\mathcal{F}$ over $S$, a subgroup $A \leq S$ is weakly closed in $\mathcal{F}$ if $A$ is the only subgroup in its $\mathcal{F}$ conjugacy class. Clearly, any normal subgroup in $\mathcal{F}$ is weakly closed, and any weakly closed subgroup is normal in $S$. When $A$ is weakly closed in $\mathcal{F}$, then we define $\mathcal{F} / A$ to be the fusion system over $S / A$ where

$$
\operatorname{Hom}_{\mathcal{F} / A}(P / A, Q / A)=\left\{f / A \mid f \in \operatorname{Hom}_{\mathcal{F}}(P, Q)\right\} .
$$

Lemma 2.1. Fix a saturated fusion system $\mathcal{F}$ over a p-group $S$. Then for any subgroup $A \triangleleft S$ which is weakly $\mathcal{F}$-closed in $S, \mathcal{F} / A$ is a saturated fusion system over $S / A$.

Proof. This is shown, for example, in [O2, Lemma 2.6]. But since it plays a central role in this paper, we repeat the proof here.

Proof of (II): Fix $\varphi \in \operatorname{Hom}_{\mathcal{F} / A}(P / A, S / A)$ such that $\operatorname{Im}(\varphi)$ is fully centralized in $\mathcal{F} / A$. Set $P^{\prime} / A=\varphi(P / A)$, and

$$
N_{\varphi}=\left\{g \in N_{S / A}(P / A) \mid \varphi c_{g} \varphi^{-1} \in \operatorname{Aut}_{S / A}\left(P^{\prime} / A\right)\right\} \text {. }
$$


Choose $Q \leq S$ which is $\mathcal{F}$-conjugate to $P$ and $P^{\prime}$ and fully normalized in $\mathcal{F}$. By [BLO2, Proposition A.2(b)], there is

$$
\chi \in \operatorname{Hom}_{\mathcal{F}}\left(N_{S}\left(P^{\prime}\right), N_{S}(Q)\right)
$$

such that $\chi\left(P^{\prime}\right)=Q$. Let $\bar{\chi} \in \operatorname{Hom}_{\mathcal{F} / A}\left(N_{S / A}\left(P^{\prime} / A\right), N_{S / A}(Q / A)\right)$ be the induced homomorphism. Thus $\bar{\chi}\left(C_{S / A}\left(P^{\prime} / A\right)\right)=C_{S / A}(Q / A)$ since $P^{\prime} / A$ is fully centralized, and this restricts to an isomorphism $C_{S}\left(P^{\prime}\right) / A \cong C_{S}(Q) / A$. We have now proven

$$
Q \text { fully normalized in } \mathcal{F} \Longrightarrow Q / A \text { fully centralized in } \mathcal{F} / A \text {. }
$$

Set $\psi=\bar{\chi} \circ \varphi \in \operatorname{Iso}_{\mathcal{F} / A}(P / A, Q / A)$ for short, and choose a lifting $\widetilde{\psi}_{0} \in \operatorname{Iso}_{\mathcal{F}}(P, Q)$ of $\psi$. Consider the subgroups

$$
\widetilde{N}_{\psi} / A=N_{\psi}=\left\{g \in N_{S / A}(P / A) \mid \psi c_{g} \psi^{-1} \in \operatorname{Aut}_{S / A}(Q / A)\right\}
$$

and

$$
K_{Q}=\operatorname{Ker}\left[\operatorname{Aut}_{\mathcal{F}}(Q) \longrightarrow \operatorname{Aut}_{\mathcal{F} / A}(Q / A)\right] .
$$

Thus $\widetilde{\psi}_{0} \operatorname{Aut}_{\widetilde{N}_{\psi}}(P) \widetilde{\psi}_{0}^{-1} \leq \operatorname{Aut}_{S}(Q) \cdot K_{Q}$. Since $Q$ is fully normalized in $\mathcal{F}, K_{Q} \triangleleft$ $\operatorname{Aut}_{S}(Q) \cdot K_{Q}$ and $\operatorname{Aut}_{S}(Q) \in \operatorname{Syl}_{p}\left(\operatorname{Aut}_{S}(Q) \cdot K_{Q}\right)$, and hence all Sylow $p$-subgroups of $\operatorname{Aut}_{S}(Q) \cdot K_{Q}$ are conjugate by elements of $K_{Q}$. In particular, there is $\omega \in K_{Q}$ such that

$$
\left(\omega \widetilde{\psi}_{0}\right) \operatorname{Aut}_{\widetilde{N}_{\psi}}(P)\left(\omega \widetilde{\psi}_{0}\right)^{-1} \leq \operatorname{Aut}_{S}(Q)
$$

Set $\widetilde{\psi}=\omega \widetilde{\psi_{0}}$. This is also a lifting of $\psi$ since $\omega \in K_{Q}\left(\omega / A=\operatorname{Id}_{Q / A}\right)$; and by $(2)$,

$$
\widetilde{N}_{\psi} \leq N_{\widetilde{\psi}} \stackrel{\text { def }}{=}\left\{g \in N_{S}(P) \mid \widetilde{\psi} c_{g} \widetilde{\psi}^{-1} \in \operatorname{Aut}_{S}(Q)\right\} \text {. }
$$

Since $Q$ is fully centralized in $\mathcal{F}$ (by axiom $(\mathrm{I}$ ) for $\mathcal{F}$ ), axiom (II) for $\mathcal{F}$ now implies that $\widetilde{\psi}$ extends to a morphism $\widehat{\psi} \in \operatorname{Hom}_{\mathcal{F}}\left(\widetilde{N}_{\psi}, N_{S}(Q)\right)$, and hence that $\psi=\bar{\chi} \circ \varphi$ extends to

$$
\bar{\psi} \in \operatorname{Hom}_{\mathcal{F} / A}\left(N_{\psi}, N_{S / A}(Q / A)\right) .
$$

We claim that $\bar{\psi}\left(N_{\varphi}\right) \leq \operatorname{Im}(\bar{\chi})$. To see this, fix $g \in N_{\varphi}$, and let $h \in N_{S / A}\left(P^{\prime} / A\right)$ be such that $\varphi c_{g} \varphi^{-1}=c_{h}$. Then

$$
c_{\bar{\psi}(g)}=\psi c_{g} \psi^{-1}=\bar{\chi} c_{h} \bar{\chi}^{-1}=c_{\bar{\chi}(h)} \in \operatorname{Aut}(Q / A),
$$

so $\bar{\psi}(g) \in \bar{\chi}(h) \cdot C_{S / A}(Q / A)$. We have already seen that $C_{S / A}(Q / A)=\bar{\chi}\left(C_{S / A}\left(P^{\prime} / A\right)\right)$, and this finishes the proof that $\bar{\psi}(g) \in \operatorname{Im}(\bar{\chi})$. Thus there is

$$
\bar{\varphi} \in \operatorname{Hom}_{\mathcal{F} / A}\left(N_{\varphi}, N_{S / A}\left(P^{\prime} / A\right)\right)
$$

such that $\bar{\chi} \circ \bar{\varphi}=\bar{\psi}$, and $\left.\bar{\varphi}\right|_{P / A}=\varphi$. This finishes the proof of condition (II) for $\mathcal{F} / A$.

Proof of $(\mathbf{I})$ : Assume $P / A$ is fully normalized in $\mathcal{F} / A$. Since $N_{S / A}\left(P^{\prime} / A\right)=N_{S}\left(P^{\prime}\right) / A$ for all $P^{\prime}$ in the same $\mathcal{F}$-conjugacy class, $P$ is also fully normalized in $\mathcal{F}$. Then $P / A$ is fully centralized in $\mathcal{F} / A$ by (1). $\operatorname{Also} \operatorname{Aut}_{S / A}(P / A) \in \operatorname{Syl}_{p}\left(\operatorname{Aut}_{\mathcal{F} / A}(P / A)\right)$ since $\operatorname{Aut}_{S}(P) \in \operatorname{Syl}_{p}\left(\operatorname{Aut}_{\mathcal{F}}(P)\right.$ ) (by condition $(\mathrm{I})$ again for $\mathcal{F}$ ), and since a surjection of finite groups sends Sylow subgroups onto Sylow subgroups.

Whenever $(S, \mathcal{F}, \mathcal{L})$ is a $p$-local finite group and $A \triangleleft S$ is normal in $\mathcal{F}$, we let $\mathcal{L} / A$ be the category whose objects are the subgroups $P / A \leq S / A$ such that $P$ is $\mathcal{F}$-centric, and where

$$
\operatorname{Mor}_{\mathcal{L} / A}(P / A, Q / A)=\operatorname{Mor}_{\mathcal{L}}(P, Q) / A
$$


Here, $g \in A$ acts on $\operatorname{Mor}_{\mathcal{L}}(P, Q)$ via composition with $\delta_{P}(g) \in \operatorname{Aut}_{\mathcal{L}}(P)$. Let $(\mathcal{L} / A)^{c}$ be the subcategory of $\mathcal{L} / A$ whose objects are the $\mathcal{F} / A$-centric subgroups of $S / A$. We will define $(\mathcal{L} / A)_{*}^{c}$ to be a certain quotient category of $(\mathcal{L} / A)^{c}$.

Lemma 2.2. Fix a p-local finite group $(S, \mathcal{F}, \mathcal{L})$, and assume $A \triangleleft S$ is normal in $\mathcal{F}$. Let $\mathcal{F} / A$ be the induced fusion system over $S / A$, and let $(\mathcal{L} / A)^{c}$ be defined as above. Then for each $\mathcal{F} / A$-centric subgroup $P / A \leq S / A, P$ is $\mathcal{F}$-centric, and there is a unique subgroup $E_{0}(P) \leq \operatorname{Aut}_{\mathcal{L} / A}(P / A)$ of order prime to $p$ such that

$$
C_{\text {Aut }_{\mathcal{L} / A}(P / A)}(P / A)=E_{0}(P) \times Z(P / A) .
$$

Let $(\mathcal{L} / A)_{*}^{c}$ be the category whose objects are the $\mathcal{F} / A$-centric subgroups of $S / A$, and where

$$
\operatorname{Mor}_{(\mathcal{L} / A)_{*}^{c}}(P / A, Q / A)=\operatorname{Mor}_{\mathcal{L} / A}(P / A, Q / A) / E_{0}(P) .
$$

Then $(\mathcal{L} / A)_{*}^{c}$ is a well defined category, and is a centric linking system associated to $\mathcal{F} / A$.

Proof. Fix an $\mathcal{F} / A$-centric subgroup $P / A \leq S / A$. For all $P^{\prime} / A$ which is $\mathcal{F} / A$-conjugate to $P / A, P^{\prime}$ is $\mathcal{F}$-conjugate to $P$, and $C_{S}\left(P^{\prime}\right) / A \leq C_{S / A}\left(P^{\prime} / A\right) \leq P^{\prime} / A$. Thus $C_{S}\left(P^{\prime}\right) \leq$ $P^{\prime}$ for all such $P^{\prime}$, and this shows that $P$ is $\mathcal{F}$-centric. In particular, $P \in \mathrm{Ob}(\mathcal{L})$.

Set

$$
K_{P}^{\prime}=\operatorname{Ker}\left[\operatorname{Aut}_{\mathcal{L} / A}(P / A) \longrightarrow \operatorname{Aut}_{\mathcal{F} / A}(P / A)\right],
$$

and let $\widehat{\delta}_{P / A}$ denote the homomorphism

$$
\begin{gathered}
\widehat{\delta}_{P / A}=\delta_{P, P} / A: N_{S / A}(P / A) \\
=N_{S}(P) / A
\end{gathered} \longrightarrow \begin{gathered}
\operatorname{Aut}_{\mathcal{L} / A}(P / A) \\
=\operatorname{Aut}_{\mathcal{L}}(P) / A
\end{gathered}
$$

If $P$ is fully normalized in $\mathcal{F}$, then

$$
\begin{aligned}
\operatorname{Aut}_{S}(P) \in \operatorname{Syl}_{p}\left(\operatorname{Aut}_{\mathcal{F}}(P)\right) \Longrightarrow & \delta_{P, P}\left(N_{S}(P)\right) \in \operatorname{Syl}_{p}\left(\operatorname{Aut}_{\mathcal{L}}(P)\right) \\
& \Longrightarrow \widehat{\delta}_{P / A}\left(N_{S / A}(P / A)\right) \in \operatorname{Syl}_{p}\left(\operatorname{Aut}_{\mathcal{L} / A}(P / A)\right) .
\end{aligned}
$$

Hence by Lemma $1.9(\mathrm{~b}), K_{P}^{\prime} \cap \widehat{\delta}_{P / A}\left(N_{S / A}(P / A)\right)=\widehat{\delta}_{P / A}(Z(P / A))$ is a Sylow $p$-subgroup of $K_{P}^{\prime}$. Since $|Z(P / A)|$ and $\left|K_{P}^{\prime}\right|$ are both invariant under $\mathcal{F}$-conjugacy, this implies that $\widehat{\delta}_{P / A}(Z(P / A)) \in \operatorname{Syl}_{p}\left(K_{P}^{\prime}\right)$ whether or not $P$ is fully normalized.

For each $f \in \operatorname{Aut}_{\mathcal{L}}(P)$ and each $g \in P$,

$$
\delta_{P}(\pi(f)(g))=f \circ \delta_{P}(g) \circ f^{-1}
$$

by axiom $(\mathrm{C})$ for $\mathcal{L}$. Upon passing to the quotient group $\operatorname{Aut}_{\mathcal{L} / A}(P / A)$, this shows that each element of $K_{P}^{\prime}$ centralizes the subgroup $\widehat{\delta}_{P / A}(P / A)$. In particular, the Sylow $p$-subgroup $\widehat{\delta}_{P / A}(Z(P / A))$ is central in $K_{P}^{\prime}$, and hence

$$
K_{P}^{\prime}=E_{0}(P) \times Z(P / A)
$$

for a unique subgroup $E_{0}(P) \leq \operatorname{Aut}_{\mathcal{L} / A}(P / A)$.

Now define $(\mathcal{L} / A)_{*}^{c}$ to be the category whose objects are the $\mathcal{F} / A$-centric subgroups of $S / A$, and where

$$
\operatorname{Mor}_{(\mathcal{L} / A)_{*}^{*}}(P / A, Q / A)=\operatorname{Mor}_{\mathcal{L} / A}(P / A, Q / A) / E_{0}(P) .
$$

To see that composition is well defined, we must show, for each $f \in \operatorname{Mor}_{\mathcal{L} / A}(P / A, Q / A)$ between $\mathcal{F} / A$-centric subgroups, that

$$
E_{0}(Q) \circ f \subseteq f \circ E_{0}(P) .
$$


For each $\alpha \in E_{0}(Q)$, there is by Lemma 1.7(a) (applied to $\mathcal{L}$ ) a unique morphism $\beta \in \operatorname{Aut}_{\mathcal{L} / A}(P / A)$ such that $\alpha \circ f=f \circ \beta$, and $\beta \in E_{0}(P)$ since the induced square in $\mathcal{F} / A$ must commute.

This shows that $(\mathcal{L} / A)_{*}^{c}$ is a well defined category. The distinguished monomorphisms

$$
\delta_{P / A}: P / A \longrightarrow \operatorname{Aut}_{\mathcal{L} / A}(P / A)=\operatorname{Aut}_{\mathcal{L}}(P) / A
$$

are induced by the distinguished monomorphisms $\delta_{P}$ for $\mathcal{L}$. Conditions $(\mathrm{B})$ and $(\mathrm{C})$ for $(\mathcal{L} / A)^{c}$ follow directly from the corresponding conditions for $\mathcal{L}$. It remains to prove condition (A), and this follows by (3) and (4).

Note that by Proposition A.10, whenever $(S, \mathcal{F}, \mathcal{L})$ is a $p$-local finite group with normal $p$-subgroup $A \triangleleft \mathcal{F}$, then the sequence

$$
B A \longrightarrow|\mathcal{L}| \longrightarrow|\mathcal{L} / A|
$$

is a homotopy fibration sequence.

It is easy to construct examples of linking systems $\mathcal{L}$ with normal subgroup $A$ for which $(\mathcal{L} / A)^{c}$ is not a centric linking system. As a rather trivial example, set $p=2$, fix a finite group $H$ of even order, and set $G=A_{4} \times H$. Let $A \triangleleft G$ be the normal subgroup of order 4 in $A_{4}$, and fix Sylow subgroups $S_{0} \in \operatorname{Syl}_{2}(H)$ and $S=A \times S_{0} \in \operatorname{Syl}_{2}(G)$. Let $\mathcal{F}=\mathcal{F}_{S}(G), \mathcal{L}=\mathcal{L}_{S}^{c}(G)$, and $\mathcal{L}_{0}=\mathcal{L}_{S_{0}}^{c}(H)$. Then $A$ is normal in $\mathcal{F}$. For any $P \leq S$ which is $\mathcal{F}$-centric and contains $A, P=A \times P_{0}$ for some $P_{0} \leq S_{0}$, $\operatorname{Aut}_{\mathcal{L}}(P)=A_{4} \times \operatorname{Aut}_{\mathcal{L}_{0}}\left(P_{0}\right)$, and hence $\operatorname{Aut}_{\mathcal{L} / A}(P / A) \cong C_{3} \times \operatorname{Aut}_{\mathcal{L}_{0}}\left(P_{0}\right)$. Thus $(\mathcal{L} / A)^{c}$ is not a linking system.

Note also, in the above example, that the kernel of the map from $\operatorname{Aut}_{\mathcal{F}}(P)$ to $\operatorname{Aut}_{\mathcal{F} / A}(P / A)$ is not a $p$-group for any $P$ containing $A$. This helps to motivate the following general criterion for $\mathcal{L} / A$ to be a linking system.

Proposition 2.3. Let $(S, \mathcal{F}, \mathcal{L})$ be a finite p-group, and assume $A \triangleleft \mathcal{F}$. Then $(\mathcal{L} / A)^{c}$ is a linking system associated to $\mathcal{F} / A$ if and only if $\operatorname{Ker}\left[\operatorname{Aut}_{\mathcal{F}}(P) \longrightarrow \operatorname{Aut}_{\mathcal{F} / A}(P / A)\right]$ is a p-group for all $P \leq S$ such that $P \geq A$ and $P / A$ is $\mathcal{F} / A$-centric.

Proof. Let $\mathcal{P}$ be the set of all subgroups $P \leq S$ such that $P \geq A$ and $P / A$ is $\mathcal{F} / A$ centric. Consider the following subgroups for all $P \in \mathcal{P}$ :

$$
\begin{aligned}
& K_{P}=\operatorname{Ker}\left[\operatorname{Aut}_{\mathcal{F}}(P) \longrightarrow \operatorname{Aut}_{\mathcal{F} / A}(P / A)\right] \\
& \left.K_{P}^{\prime}=\operatorname{Ker}_{\operatorname{Aut}_{\mathcal{L} / A}}(P / A) \longrightarrow \operatorname{Aut}_{\mathcal{F} / A}(P / A)\right] \text {. }
\end{aligned}
$$

By Lemma 2.2, $K_{P}^{\prime}=E_{0}(P) \times Z(P / A)$ for some subgroup $E_{0}(P)$ of order prime to $p$, and $(\mathcal{L} / A)^{c}$ is a linking system associated to $\mathcal{F} / A$ if and only if $K_{P}^{\prime}$ is a $p$-group for all $P \in \mathcal{P}$.

Consider the diagram

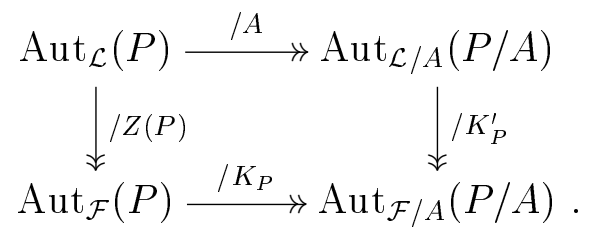

Here, in all cases, "/ $H$ " means dividing out by the subgroup $H$. Since $A$ and $Z(P)$ are both $p$-groups, this shows that $K_{P}$ is a $p$-group if and only if $K_{P}^{\prime}$ is a $p$-group, and thus $(\mathcal{L} / A)^{c}$ is a linking system if and only if $K_{P}$ is a $p$-group for all $P \in \mathcal{P}$. 
The following proposition describes one more very simple way to construct such examples.

Proposition 2.4. Fix a finite group $G$ and Sylow subgroup $S \in \operatorname{Syl}_{p}(G)$. Assume there is a normal p-subgroup $A \triangleleft G$ which is centric in $G$; i.e., $C_{G}(A)=Z(A)$. Let $\mathcal{L}_{\bar{S}}^{\geq A}(G) \subseteq \mathcal{L}_{S}^{c}(G)$ be the full subcategory with objects those $P \leq S$ containing $A$. Then $A$ is normal in $\mathcal{F}_{S}(G)$, and $\mathcal{L}_{\bar{S}}^{>A}(G) / A \cong \mathcal{T}_{S / A}(G / A)$.

Proof. Since $A$ is centric in $G$, so is every subgroup which contains $A$. Thus $C_{G}(P)=$ $Z(P)$ for every $P \in \mathrm{Ob}\left(\mathcal{L}_{\bar{S}}^{\geq A}(G)\right)$, and so $\mathcal{L}_{\bar{S}}^{\geq A}(G)$ is a full subcategory of the transporter category $\mathcal{T}_{S}(G)$. It follows that $\mathcal{L}_{\bar{S}}^{\geq A}(G) / A \cong \mathcal{T}_{\bar{S}}^{\geq A}(G) \cong \mathcal{T}_{S / A}(G / A)$.

One easily finds examples of groups whose (centric) transporter category is not a linking category. For example, there are subgroups $P \leq A_{7}$ such that $P \cong C_{2}^{2}$, and $C_{A_{7}}(P) \cong C_{2}^{2} \times C_{3}$. Thus $P$ is 2-centric in $A_{7}$, but for $S \in \operatorname{Syl}_{2}\left(A_{7}\right), \mathcal{L}_{S}^{c}\left(A_{7}\right)$ is not a full subcategory of $\mathcal{T}_{S}\left(A_{7}\right)$. So if we set $G=C_{2}^{7} \rtimes A_{7}$, where $A_{7}$ acts on $A=C_{2}^{7}$ by permuting a basis, then $\left(\mathcal{L}_{S}^{c}(G) / A\right)^{c}$ is not a linking system for $G / A \cong A_{7}$.

\section{TRANSPORTER SYSTEMS}

In the last section, we saw that for a centric linking system $\mathcal{L}$ with normal subgroup $A$, the quotient category $(\mathcal{L} / A)^{c}$ need not be a linking system associated to any fusion system. This motivates us to define what we call "transporter systems": categories with extra structures satisfying some properties similar to those of the transporter categories associated to finite groups, but without necessarily having such a group.

Recall (Definition 1.1) that for any finite group $G$ and any $S \in \operatorname{Syl}_{p}(G), \mathcal{T}_{S}(G)$ denotes the category with objects the subgroups of $G$, and with morphism sets the transporter sets $N_{G}(P, Q)$. For any set $\mathcal{H}$ of subgroups of $S$, we let $\mathcal{T}_{\mathcal{H}}(G)$ be the full subcategory of $\mathcal{T}_{S}(G)$ with object set $\mathcal{H}$.

Definition 3.1. Let $\mathcal{F}$ be a fusion system over a p-group $S$. A transporter system associated to a fusion system $\mathcal{F}$ is a nonempty finite category $\mathcal{T}$, together with a pair of functors

$$
\mathcal{T}_{\mathrm{Ob}(\mathcal{T})}(S) \stackrel{\varepsilon}{\longrightarrow} \mathcal{T} \stackrel{\rho}{\longrightarrow} \mathcal{F}
$$

satisfying the following conditions:

(A1) $\mathrm{Ob}(\mathcal{T}) \subseteq \mathrm{Ob}(\mathcal{F})$, and $\mathrm{Ob}(\mathcal{T})$ is closed under $\mathcal{F}$-conjugacy and overgroups. Also, $\varepsilon$ is the identity on objects and $\rho$ is the inclusion on objects.

(A2) For each $P, Q \in \mathrm{Ob}(\mathcal{T})$, the kernel

$$
E(P) \stackrel{\text { def }}{=} \operatorname{Ker}\left[\rho_{P, P}: \operatorname{Aut}_{\mathcal{T}}(P) \longrightarrow \operatorname{Aut}_{\mathcal{F}}(P)\right]
$$

acts freely on $\operatorname{Mor}_{\mathcal{T}}(P, Q)$ by right composition, and $\rho_{P, Q}$ is the orbit map for this action. Also, $E(Q)$ acts freely on $\operatorname{Mor}_{\mathcal{T}}(P, Q)$ by left composition.

(B) For each $P, Q \in \mathrm{Ob}(\mathcal{T}), \varepsilon_{P, Q}: N_{S}(P, Q) \longrightarrow \operatorname{Mor}_{\mathcal{T}}(P, Q)$ is injective, and the composite $\rho_{P, Q} \circ \varepsilon_{P, Q}$ sends $g \in N_{S}(P, Q)$ to $c_{g} \in \operatorname{Hom}_{\mathcal{F}}(P, Q)$. 
(C) For all $\varphi \in \operatorname{Mor}_{\mathcal{T}}(P, Q)$ and all $g \in P$, the diagram

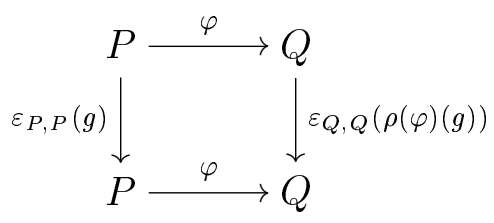

commutes in $\mathcal{T}$.

(I) $\varepsilon_{S, S}(S) \in \operatorname{Syl}_{p}\left(\operatorname{Aut}_{\mathcal{T}}(S)\right)$.

(II) Let $\varphi \in \operatorname{Iso}_{\mathcal{T}}(P, Q), P \triangleleft \bar{P} \leq S$, and $Q \triangleleft \bar{Q} \leq S$ be such that $\varphi \circ \varepsilon_{P, P}(\bar{P}) \circ \varphi^{-1} \leq$ $\varepsilon_{Q, Q}(\bar{Q})$. Then there is some $\bar{\varphi} \in \operatorname{Mor}_{\mathcal{T}}(\bar{P}, \bar{Q})$ such that $\bar{\varphi} \circ \varepsilon_{P, \bar{P}}(1)=\varepsilon_{Q, \bar{Q}}(1) \circ \varphi$.

We will often write " $(S, \mathcal{F}, \mathcal{T})$ is a transporter system" to mean that $\mathcal{T}$ is a transporter system associated to the fusion system $\mathcal{F}$ over the $p$-group $S$. The above axioms are clearly labelled to show their connection with axioms (A), (B), and (C) of a linking system, and axioms (I) and (II) of a saturated fusion system. Note, however, that the concepts of fully normalized and fully centralized subgroups do not appear in the axioms of a transporter system - which does help simplify some of our proofs that if one category is a transporter system then another one is too.

For any transporter system $(S, \mathcal{F}, \mathcal{T})$, axiom (A2) implies that the functor $\rho: \mathcal{T} \rightarrow \mathcal{F}$ is "source regular" in the sense of Definition A.5. So by Lemma A.6, a morphism in $\mathcal{T}$ is an isomorphism if and only if its image in $\mathcal{F}$ is an isomorphism. In particular, $\mathcal{T}$ is an EI category (all endomorphisms are automorphisms) since $\mathcal{F}$ is one.

We will show in Proposition 3.5 that transporter categories of finite groups, linking systems associated to saturated fusion systems, and (more generally) categories of the form $\mathcal{L} / A$ when $\mathcal{L}$ is a linking system and $A$ a normal subgroup, are all examples of transporter systems. These examples provided our main motivation for the above definition, and the axioms for a transporter system are clearly related to those for fusion and linking systems.

We will prove soon, as Proposition 3.4(a), the following stronger form of axiom (I), which is more closely analogous to the axiom (I) in Definition 1.3:

$\left(\mathrm{I}^{\prime}\right)$ If $P$ is fully normalized in $\mathcal{F}$, then $\varepsilon_{P, P}\left(N_{S}(P)\right) \in \operatorname{Syl}_{p}\left(\operatorname{Aut}_{\mathcal{T}}(P)\right)$.

The weaker axiom (I) we use here is motivated by the alternative set of axioms for a saturated fusion system due to Radu Stancu [St].

For all $P \leq Q \leq S$ objects in $\mathcal{T}$, we set $\iota_{P, Q}=\varepsilon_{P, Q}(1)$, and think of these as the inclusion morphisms in $\mathcal{T}$. By condition (B), $\rho$ sends inclusions in $\mathcal{T}$ to inclusions in $\mathcal{F}$. Whenever $P_{0} \leq P \leq S$ and $Q_{0} \leq Q \leq S$ are in $\operatorname{Ob}(\mathcal{T})$, and

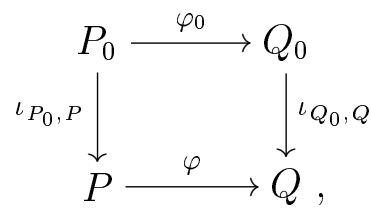

is a commutative square in $\mathcal{T}$, we say that $\varphi_{0}$ is a restriction of $\varphi$ (and sometimes write $\left.\varphi\right|_{P_{0}, Q_{0}}=\varphi_{0}$ ), and also that $\varphi$ is an extension of $\varphi_{0}$. Thus axiom (II) gives conditions under which a morphism can be extended.

This terminology suggests that the restriction $\left.\varphi\right|_{P_{0}, Q_{0}}$ should always exist and be unique, whenever $P_{0}$ and $Q_{0}$ are objects in $\mathcal{T}$ and $\rho(\varphi)\left(P_{0}\right) \leq Q_{0}$. This is shown in the 
following lifting lemma, which is the analog for transporter systems of Lemma 1.7(a). We will see later (Lemma 3.8) that extensions are also unique when they exist.

Lemma 3.2. Let $(S, \mathcal{F}, \mathcal{T})$ be a transporter system, and let $\rho: \mathcal{T} \longrightarrow \mathcal{F}$ be the projection functor.

(a) Fix morphisms $\varphi \in \operatorname{Hom}_{\mathcal{F}}(P, Q)$ and $\psi \in \operatorname{Hom}_{\mathcal{F}}(Q, R)$, where $P, Q, R \in \operatorname{Ob}(\mathcal{T})$. Then for any pair of liftings $\widetilde{\psi} \in \rho_{Q, R}^{-1}(\psi)$ and $\widetilde{\psi \varphi} \in \rho_{P, R}^{-1}(\psi \varphi)$, there is a unique lifting $\widetilde{\varphi} \in \rho_{P, Q}^{-1}(\varphi)$ such that $\widetilde{\psi} \circ \widetilde{\varphi}=\widetilde{\psi \varphi}$.

(b) All morphisms in $\mathcal{T}$ are monomorphisms in the categorical sense. In other words, for all $P, Q, R \in \mathrm{Ob}(\mathcal{T})$ and all $\varphi_{1}, \varphi_{2} \in \operatorname{Mor}_{\mathcal{T}}(P, Q)$ and $\psi \in \operatorname{Mor}_{\mathcal{T}}(Q, R)$, $\psi \circ \varphi_{1}=\psi \circ \varphi_{2}$ implies $\varphi_{1}=\varphi_{2}$.

(c) For every morphism $\varphi \in \operatorname{Mor}_{\mathcal{T}}(P, Q)$, and every $P_{0}, Q_{0} \in \operatorname{Ob}(\mathcal{T})$ such that $P_{0} \leq$ $P, Q_{0} \leq Q$, and $\rho(\varphi)\left(P_{0}\right) \leq Q_{0}$, there is a unique morphism $\varphi_{0} \in \operatorname{Mor}_{\mathcal{T}}\left(P_{0}, Q_{0}\right)$ such that $\varphi \circ \iota_{P_{0}, P}=\iota_{Q_{0}, Q} \circ \varphi_{0}$. In particular, every morphism in $\mathcal{T}$ is the composite of an isomorphism followed by an inclusion.

Proof. Since morphisms in $\mathcal{F}$ are all group monomorphisms, they are also monomorphisms in the categorical sense. Hence points (a) and (b) are special cases of Lemma A.7(a,b).

In the situation of (c), by definition of a fusion system, $\left.\rho(\varphi)\right|_{P_{0}, Q_{0}} \in \operatorname{Hom}_{\mathcal{F}}\left(P_{0}, Q_{0}\right)$. So the result follows from (a), with $P, Q, R$ replaced by $P_{0}, Q_{0}, Q, \widetilde{\psi \varphi}$ by $\varphi \circ \iota_{P_{0}, P}$, etc. The last statement is the special case where $P_{0}=P$ and $Q_{0}=\rho(\varphi)(P)$.

The following technical lemma can be thought of as a converse to axiom (II). It shows that the condition for extending a morphism, which is sufficent by axiom (II), is also necessary.

Lemma 3.3. Fix a transporter system $(S, \mathcal{F}, \mathcal{T})$, and objects $P \triangleleft \bar{P}$ and $Q \leq \bar{Q}$ of $\mathcal{T}$. If $\bar{\varphi} \in \operatorname{Mor}_{\mathcal{T}}(\bar{P}, \bar{Q})$ is an extension of $\varphi \in \operatorname{Iso}_{\mathcal{T}}(P, Q)$, then

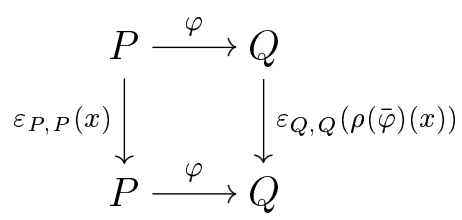

commutes in $\mathcal{T}$ for all $x \in \bar{P}$.

Proof. By axiom (C), the following square commutes in $\mathcal{T}$ :

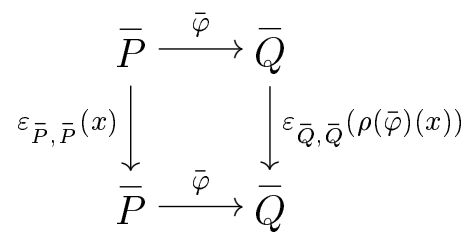

Each morphism in (1) is the restriction of the corresponding morphism in (2). So square (1) also commutes by the uniqueness of restriction morphisms (Lemma 3.2(c)).

We next prove that axiom (I) can be replaced by the stronger axiom ( $\left.\mathrm{I}^{\prime}\right)$ stated above. 
Proposition 3.4. The following hold for any transporter system $(S, \mathcal{F}, \mathcal{T})$ and any subgroup $P \leq S$.

(a) $P$ is fully normalized in $\mathcal{F}$ if and only if $\varepsilon_{P, P}\left(N_{S}(P)\right) \in \operatorname{Syl}_{p}\left(\operatorname{Aut}_{\mathcal{T}}(P)\right)$.

(b) $P$ is fully centralized in $\mathcal{F}$ if and only if $\varepsilon_{P, P}\left(C_{S}(P)\right) \in \operatorname{Syl}_{p}(E(P))$.

Proof. The "if" part in both (a) and (b) is clear, since $\left|\operatorname{Aut}_{\mathcal{T}}(P)\right|$ and $|E(P)|$ depend only on the $\mathcal{F}$-conjugacy class of $P$.

(a) Suppose otherwise: that $\mathcal{T}$ does not satisfy axiom $\left(\mathrm{I}^{\prime}\right)$. Let $P$ be a maximal counterexample. Thus $P$ is fully normalized, and $\varepsilon_{P, P}\left(N_{S}(P)\right)$ is not a $p$-Sylow subgroup of $\operatorname{Aut}_{\mathcal{T}}(P)$. Since axiom $\left(\mathrm{I}^{\prime}\right)$ holds for the group $S$ by assumption, $P \supsetneqq S$, and so $P \supsetneqq N_{S}(P)$. Choose $Q \in \operatorname{Syl}_{p}\left(\operatorname{Aut}_{\mathcal{T}}(P)\right)$ such that $\varepsilon_{P, P}\left(N_{S}(P)\right) \leq Q$. We know that $\varepsilon_{P, P}\left(N_{S}(P)\right) \neq Q$, so $\varepsilon_{P, P}\left(N_{S}(P)\right) \supsetneqq Q_{0} \stackrel{\text { def }}{=} N_{Q}\left(\varepsilon_{P, P}\left(N_{S}(P)\right)\right)$. We thus have strict inclusions of $p$-subgroups of $\operatorname{Aut}_{\mathcal{T}}(P)$ :

$$
\varepsilon_{P, P}(P) \supsetneqq \varepsilon_{P, P}\left(N_{S}(P)\right) \triangleleft Q_{0}
$$

Pick any morphism $\varphi \in Q_{0}$ not in $\varepsilon_{P, P}\left(N_{S}(P)\right)$. Then $\varphi^{-1} \circ \varepsilon_{P, P}(x) \circ \varphi \in \varepsilon_{P, P}\left(N_{S}(P)\right)$ for all $x \in N_{S}(P)$ since $\varepsilon_{P, P}\left(N_{S}(P)\right) \triangleleft Q_{0}$. So by axiom (II), there is an extension $\bar{\varphi} \in \operatorname{Aut}_{\mathcal{T}}\left(N_{S}(P)\right)$ of $\varphi$; i.e., a morphism such that the following square commutes:

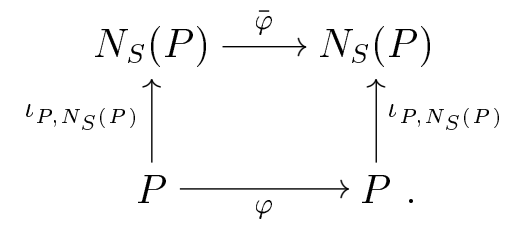

Set $|\bar{\varphi}|=p^{k} m$ where $p \nmid m$. Choose $r$ such that $r \equiv 0(\bmod m)$ and $r \equiv 1\left(\bmod p^{k}\right)$. Then $\bar{\varphi}^{r}$ has order $p^{k}$ and (since $|\varphi| \mid p^{k}$ ) is again an extension of $\varphi^{r}=\varphi$. We can thus assume that $\bar{\varphi}$ has $p$-power order.

Choose $\bar{P}$ fully normalized and $\mathcal{F}$-conjugate to $N_{S}(P)$. Let $\gamma \in \operatorname{Iso}_{\mathcal{T}}\left(N_{S}(P), \bar{P}\right)$ be any lifting of an isomorphism $\gamma^{\prime} \in \operatorname{Iso}_{\mathcal{F}}\left(N_{S}(P), \bar{P}\right)$. Since $|\bar{P}|=\left|N_{S}(P)\right|>|P|$ and $P$ is a maximal counterexample, we have $\varepsilon_{\bar{P}, \bar{P}}\left(N_{S}(\bar{P})\right) \in \operatorname{Syl}_{p}\left(\operatorname{Aut}_{\mathcal{T}}(\bar{P})\right)$. Hence $\gamma \circ \bar{\varphi} \circ \gamma^{-1}$ is conjugate to $\varepsilon_{\bar{P}, \bar{P}}(x)$ for some $x \in N_{S}(\bar{P})$, because $\bar{\varphi}$ has $p$-power order (and so does $\left.\gamma \circ \bar{\varphi} \circ \gamma^{-1}\right)$ and all $p$-Sylow subgroups are conjugate. By replacing $\gamma$ by an appropriate element of $\operatorname{Aut}_{\mathcal{T}}(\bar{P}) \circ \gamma$, we can arrange that $\gamma \circ \bar{\varphi} \circ \gamma^{-1}=\varepsilon_{\bar{P}, \bar{P}}(x)$.

Now, $\bar{\varphi} \in \operatorname{Aut}_{\mathcal{T}}(\bar{P})$ restricts to $\varphi \in \operatorname{Aut}_{\mathcal{T}}(P)$. Set $R=\rho(\gamma)(P) \leq \bar{P}$ and $\gamma_{0}=$ $\left.\gamma\right|_{P, R} \in \operatorname{Iso}_{\mathcal{T}}(P, R)$ (using Lemma 3.2(c)). Then $\gamma_{0} \varphi \gamma_{0}^{-1} \in \operatorname{Aut}_{\mathcal{T}}(R)$ is a restriction of $\varepsilon_{\bar{P}, \bar{P}}(x)$. Hence $\rho\left(\varepsilon_{\bar{P}, \bar{P}}(x)\right)=c_{x}$ restricts to an automorphism of $R$ (in $\mathcal{F}$ ), which means that $x \in N_{S}(R)$ and $\gamma_{0} \varphi \gamma_{0}^{-1}=\varepsilon_{R, R}(x)$. Also, $\left|N_{S}(R)\right| \leq\left|N_{S}(P)\right|$ since $P$ is fully normalized and $R$ is $\mathcal{F}$-conjugate to $P$, so $\rho(\gamma)\left(N_{S}(P)\right)=N_{S}(R)$, and $x=\rho(\gamma)(y)$ for some $y \in N_{S}(P)$. We thus have the following two commutative squares of isomorphisms in $\mathcal{T}$ :
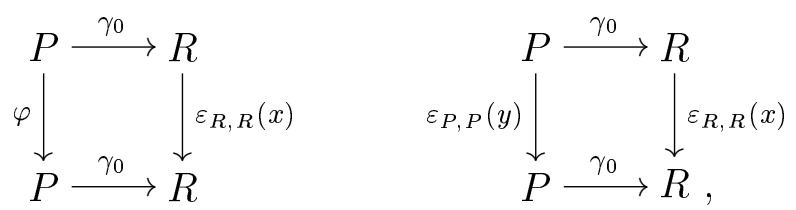

where the second commutes by Lemma 3.3. Upon comparing the two squares, we finally get $\varphi=\varepsilon_{P, P}(y)$, which contradicts our assumption that $\varphi \notin \varepsilon_{P, P}\left(N_{S}(P)\right)$. 
(b) Again fix $P \leq S$, and let $P^{\prime}$ be any subgroup which is $\mathcal{F}$-conjugate to $P$ and fully normalized in $\mathcal{F}$. Then $\varepsilon_{P^{\prime}, P^{\prime}}\left(N_{S}\left(P^{\prime}\right)\right) \in \operatorname{Syl}_{p}\left(\operatorname{Aut}_{\mathcal{T}}\left(P^{\prime}\right)\right)$ by (a), and hence $\varepsilon_{P^{\prime}, P^{\prime}}\left(C_{S}\left(P^{\prime}\right)\right)=\varepsilon_{P^{\prime}, P^{\prime}}\left(N_{S}\left(P^{\prime}\right)\right) \cap E\left(P^{\prime}\right)$ is a Sylow $p$-subgroup of $E\left(P^{\prime}\right)$ by Lemma 1.9 (b). Also, $E(P) \cong E\left(P^{\prime}\right)$, so $P$ is fully centralized if and only if $\left|C_{S}(P)\right|=\left|C_{S}\left(P^{\prime}\right)\right|$; equivalently, $\varepsilon_{P, P}\left(C_{S}(P)\right) \in \operatorname{Syl}_{p}(E(P))$.

We next check that the examples which motivated Definition 3.1 really are transporter systems.

Proposition 3.5. (a) For any p-local finite group $(S, \mathcal{F}, \mathcal{L}), \mathcal{L}$ is a transporter system associated to $\mathcal{F}$. More generally, if $A \triangleleft \mathcal{F}$ is a normal subgroup, then $\mathcal{L} / A$ is a transporter system associated to $\mathcal{F} / A$.

(b) For any finite group $G$ and any $S \in \operatorname{Syl}_{p}(G), \mathcal{T}_{S}(G)$ is a transporter system associated to $\mathcal{F}_{S}(G)$.

(c) Let $(S, \mathcal{F}, \mathcal{T})$ be a transporter system, and let $\mathcal{T}_{0} \subseteq \mathcal{T}$ be any nonempty full subcategory such that $\mathrm{Ob}\left(\mathcal{T}_{0}\right)$ is closed under $\mathcal{F}$-conjugacy and overgroups. Then $\mathcal{T}_{0}$ is also a transporter system associated to $\mathcal{F}$.

Proof. Point (c) follows immediately from Definition 3.1.

We next check point (b). For finite $G$ and $S \in \operatorname{Syl}_{p}(G)$, define

$$
\mathcal{T}(S) \stackrel{\varepsilon}{\longrightarrow} \mathcal{T}_{S}(G) \stackrel{\rho}{\longrightarrow} \mathcal{F}_{S}(G)
$$

to be the inclusion, and the functor $g \mapsto c_{g}$, respectively. The axioms of Definition 3.1 are easily checked.

It remains to prove $(\mathrm{a})$. Let $(S, \mathcal{F}, \mathcal{L})$ be a $p$-local finite group. We prove here only that $\mathcal{L}$ is a transporter system associated to $\mathcal{F}$. The last statement, that $\mathcal{L} / A$ is a transporter system for any $A \triangleleft \mathcal{F}$, will then follow as a special case of Proposition 3.11 , to be shown later.

Fix morphisms $\iota_{P} \in \operatorname{Mor}_{\mathcal{L}}(P, S)$, for all $P \in \operatorname{Ob}(\mathcal{L})$, such that $\pi\left(\iota_{P}\right)=\operatorname{incl}_{P, S} \in$ $\operatorname{Hom}_{\mathcal{F}}(P, S)$, and such that $\iota_{S}=\operatorname{Id}_{S}$. By Corollary 1.8, there is a unique functor

$$
\varepsilon: \mathcal{T}_{\mathrm{Ob}(\mathcal{L})}(S) \longrightarrow \mathcal{L}
$$

such that $\varepsilon_{P, S}(1)=\iota_{P}$ and $\left.\left(\varepsilon_{P, P}\right)\right|_{P}=\delta_{P}$ for all $P$. Furthermore, $\varepsilon_{P, Q}$ is an injection of $N_{S}(P, Q)$ into $\operatorname{Mor}_{\mathcal{L}}(P, Q)$ for all $P, Q \in \operatorname{Ob}(\mathcal{L})$ (again by Corollary 1.8). For each $P \leq Q \leq S$ such that $P, Q \in \operatorname{Ob}(\mathcal{L})$, we set $\iota_{P, Q}=\varepsilon_{P, Q}(1) \in \operatorname{Mor}_{\mathcal{L}}(P, Q)$. We think of these as the inclusion morphisms, and define restriction and extension in $\mathcal{L}$ with respect to them.

We are now ready to check that the axioms of a transporter system hold for $\mathcal{L}$.

Axioms (A1) and (C) follow immediately from axioms (A) and (C) for a linking system.

Axiom (A2) : By axiom (A) for a linking system, for any $P, Q \in \mathrm{Ob}(\mathcal{L}), E(P)=$ $\delta_{P}(Z(P))$ acts freely on $\operatorname{Mor}_{\mathcal{L}}(P, Q)$, and $\rho_{P, Q}$ is the orbit map of that action. It remains to show that $E(Q)=\delta_{Q}(Z(Q))$ acts freely on $\operatorname{Mor}_{\mathcal{L}}(P, Q)$. Assume $f \in \operatorname{Mor}_{\mathcal{L}}(P, Q)$ and $x \in Z(Q)$ are such that $\delta_{Q}(x) \circ f=f$. Then $x$ centralizes $\pi(f)(P)$, so $x=\pi(f)(y)$ for some $y \in Z(P)$ since $P$ is $\mathcal{F}$-centric, $f=\delta_{Q}(x) \circ f=f \circ \delta_{P}(y)$ by axiom $(\mathrm{C})$ for a linking system, and so $y=1$ by axiom (A) of a linking system. Thus $x=1$, and the action is free. 
Axiom (B) : For any $P, Q \in \mathrm{Ob}(\mathcal{L})$ and any $x \in N_{S}(P, Q)$,

$$
\iota_{Q} \circ \varepsilon_{P, Q}(x)=\varepsilon_{Q, S}(1) \circ \varepsilon_{P, Q}(x)=\varepsilon_{S, S}(x) \circ \varepsilon_{P, S}(1)=\delta_{S}(x) \circ \iota_{P} .
$$

Since $\pi\left(\iota_{P}\right)$ and $\pi\left(\iota_{Q}\right)$ are inclusions in $\mathcal{F}$, this shows that $\pi\left(\varepsilon_{P, Q}(x)\right)$ is the restriction to $\operatorname{Hom}_{\mathcal{F}}(P, Q)$ of $\pi\left(\delta_{S}(x)\right)$, and hence is conjugation by $x$ by axiom (B) for $\mathcal{L}$ as a linking system. Thus $\pi\left(\varepsilon_{P, Q}(x)\right)=c_{x}$, and this proves axiom $(\mathrm{B})$ for $\mathcal{L}$ as a transporter system.

Axiom $\left(\mathbf{I}^{\prime}\right)$ : Assume $P$ is fully normalized in $\mathcal{F}$. Then $\operatorname{Aut}_{S}(P) \cong N_{S}(P) / Z(P)$ is a Sylow $p$-subgroup of $\operatorname{Aut}_{\mathcal{F}}(P) \cong \operatorname{Aut}_{\mathcal{L}}(P) / \delta_{P}(Z(P))$ by axiom (I) for the saturated fusion system $\mathcal{F}$ (and axiom $(\mathrm{A})$ for $\mathcal{L}$ as a linking system), and so $\varepsilon_{P, P}\left(N_{S}(P)\right)$ is also a Sylow $p$-subgroup of $\operatorname{Aut}_{\mathcal{L}}(P)$.

Axiom (II) : Let $f \in \operatorname{Iso}_{\mathcal{L}}(P, Q)$, and $P \triangleleft \bar{P} \leq S$ and $Q \triangleleft \bar{Q} \leq S$, be such that $f \circ \varepsilon_{P, P}(\bar{P}) \circ f^{-1} \leq \varepsilon_{Q, Q}(\bar{Q})$. If $Q$ is fully centralized in $\mathcal{F}$, then axiom (II) for the saturated fusion system $\mathcal{F}$ implies that $\pi(f)$ extends to a homomorphism $\psi \in$ $\operatorname{Hom}_{\mathcal{F}}(\bar{P}, S)$, whose image must be contained in $\bar{Q}$ since $\pi(f)$ conjugates $\operatorname{Aut}_{\bar{P}}(P)$ into $\operatorname{Aut}_{\bar{Q}}(Q)$. Hence by Lemma $1.7(\mathrm{~b})$, there is $\bar{f} \in \operatorname{Mor}_{\mathcal{L}}(\bar{P}, \bar{Q})$ (not necessarily a lifting of $\psi$ ) which extends $f$.

Now assume $Q$ is not fully centralized. Choose $R$ which is $\mathcal{F}$-conjugate to $P$ and $Q$ and fully normalized in $\mathcal{F}$. Then $\varepsilon_{R, R}\left(N_{S}(R)\right)$ is a Sylow $p$-subgroup of $\operatorname{Aut}_{\mathcal{L}}(R)$ (by axiom $\left(\mathrm{I}^{\prime}\right)$ ), and hence contains every $p$-subgroup of $\operatorname{Aut}_{\mathcal{L}}(R)$ up to conjugacy. For any isomorphism $\varphi \in \operatorname{Iso}_{\mathcal{L}}(Q, R), \varphi \circ \varepsilon_{Q, Q}\left(N_{S}(Q)\right) \circ \varphi^{-1}$ is a $p$-subgroup of $\operatorname{Aut}_{\mathcal{L}}(R)$, so there is $\chi \in \operatorname{Aut}_{\mathcal{L}}(R)$ such that $\varphi^{\prime} \stackrel{\text { def }}{=} \chi \circ \varphi$ conjugates $\varepsilon_{Q, Q}\left(N_{S}(Q)\right)$ into $\varepsilon_{R, R}\left(N_{S}(R)\right)$. Since $R$ is fully centralized, the result of the last paragraph implies that there are morphisms $\bar{f}^{\prime} \in \operatorname{Mor}_{\mathcal{L}}\left(\bar{P}, N_{S}(R)\right)$ and $\bar{\varphi} \in \operatorname{Mor}_{\mathcal{L}}\left(\bar{Q}, N_{S}(R)\right)$ such that $\left.\bar{f}^{\prime}\right|_{P, R}=\varphi^{\prime} \circ f$ and $\left.\bar{\varphi}\right|_{Q, R}=\varphi^{\prime}$. By axiom (C) for a linking system,

$$
\bar{f}^{\prime} \circ \delta_{\bar{P}}(\bar{P}) \circ \bar{f}^{\prime-1}=\delta_{N_{S}(R)}\left(\pi\left(\bar{f}^{\prime}\right)(\bar{P})\right) \quad \text { and } \quad \bar{\varphi} \circ \delta_{\bar{Q}}(\bar{Q}) \circ \bar{\varphi}^{-1}=\delta_{N_{S}(R)}(\pi(\bar{\varphi})(\bar{Q})) .
$$

After restriction, this shows that in $\operatorname{Aut}_{\mathcal{T}}(R)$,

$$
\begin{aligned}
\varepsilon_{R, R}\left(\pi\left(\bar{f}^{\prime}\right)(\bar{P})\right)=\left(\varphi^{\prime} \circ f\right) \circ \varepsilon_{P, P}(\bar{P}) \circ\left(\varphi^{\prime} \circ f\right)^{-1} & \\
& \leq \varphi^{\prime} \circ \varepsilon_{Q, Q}(\bar{Q}) \circ \varphi^{\prime-1}=\varepsilon_{R, R}(\pi(\bar{\varphi})(\bar{Q})),
\end{aligned}
$$

where the inequality holds since $f \circ \varepsilon_{P, P}(\bar{P}) \circ f^{-1} \leq \varepsilon_{Q, Q}(\bar{Q})$ by assumption. Thus $\pi\left(\bar{f}^{\prime}\right)(\bar{P}) \leq \pi(\bar{\varphi})(\bar{Q})$. By definition of a fusion system, there is $\lambda \in \operatorname{Hom}_{\mathcal{F}}(\bar{P}, \bar{Q})$ such that $\pi(\bar{\varphi})=\pi\left(\bar{f}^{\prime}\right) \circ \lambda$, and so Lemma 1.7 (a) now implies that there is $\bar{f} \in \operatorname{Mor}_{\mathcal{L}}(\bar{P}, \bar{Q})$ such that $\bar{\varphi}=\bar{f}^{\prime} \circ \bar{f}$. Upon restricting these morphisms to $P$, this implies that $\varphi^{\prime} \circ f=$ $\left.\varphi^{\prime} \circ \bar{f}\right|_{P, Q}$; and hence by Lemma $1.7\left(\right.$ a) that $\left.\bar{f}\right|_{P, Q}=f$.

More generally, one can also show that any quasicentric linking system in the sense of [BCGLO1, §3]) is a transporter system.

Later, in Proposition 3.12, we prove a partial converse to Proposition 3.5(b), by showing that any transporter system which "has enough objects" in a certain sense to be made precise is a full subcategory of the transporter category of a finite group.

Transporter systems were defined associated to arbitrary fusion systems. But in fact, the conditions on the definition are sufficiently restrictive that if a transporter system $\mathcal{T}$ is associated to a fusion system $\mathcal{F}$, then $\mathcal{F}$ is saturated - at least with respect to the objects of $\mathcal{T}$. 
Proposition 3.6. Let $\mathcal{F}$ be a fusion system over a p-group $S$ (not necessarily saturated), and let $\mathcal{T}$ be a transporter system associated to $\mathcal{F}$. Then $\mathcal{F}$ is $\operatorname{Ob}(\mathcal{T})$-saturated. If $\mathcal{F}$ is also $\mathrm{Ob}(\mathcal{T})$-generated, and if $\mathrm{Ob}(\mathcal{T}) \supseteq \mathrm{Ob}\left(\mathcal{F}^{c}\right)$, then $\mathcal{F}$ is saturated. More generally, $\mathcal{F}$ is saturated if it is $\mathrm{Ob}(\mathcal{T})$-generated, and every $\mathcal{F}$-centric subgroup $P \leq S$ not in $\mathrm{Ob}(\mathcal{T})$ is $\mathcal{F}$-conjugate to some $P^{\prime}$ such that $\operatorname{Out}_{S}\left(P^{\prime}\right) \cap O_{p}\left(\operatorname{Out}_{\mathcal{F}}\left(P^{\prime}\right)\right) \neq 1$.

Proof. Assume $P \in \mathrm{Ob}(\mathcal{T})$. If $P$ is fully normalized in $\mathcal{F}$, then by Proposition 3.4(a), $\varepsilon_{P, P}\left(N_{S}(P)\right) \in \operatorname{Syl}_{p}\left(\operatorname{Aut}_{\mathcal{T}}(P)\right)$. Hence by Lemma 1.9(b), applied with $G=\operatorname{Aut}_{\mathcal{T}}(P)$ and $H=E(P), \varepsilon_{P, P}\left(C_{S}(P)\right) \in \operatorname{Syl}_{p}(E(P))$ (hence $P$ is fully centralized by Proposition 3.4(b)) and $\operatorname{Aut}_{S}(P) \in \operatorname{Syl}_{p}\left(\operatorname{Aut}_{\mathcal{F}}(P)\right)$. Thus $\mathcal{F}$ satisfies axiom (I) for the subgroup $P$.

Now fix $f \in \operatorname{Iso}_{\mathcal{F}}(P, Q)$ such that $Q$ is fully centralized in $\mathcal{F}$, and let $\varphi \in \operatorname{Iso}_{\mathcal{T}}(P, Q)$ be any isomorphism such that $\rho(\varphi)=f$. Set

$$
N_{f}=\left\{x \in N_{S}(P) \mid f \circ c_{x} \circ f^{-1} \in \operatorname{Aut}_{S}(Q)\right\} .
$$

Then $\varphi\left(\varepsilon_{P, P}\left(N_{f}\right)\right) \varphi^{-1}$ is a $p$-subgroup of $E(Q) \cdot \varepsilon_{Q, Q}\left(N_{S}(Q)\right)$. Since $Q$ is fully centralized, $\varepsilon_{Q, Q}\left(C_{S}(Q)\right) \in \operatorname{Syl}_{p}(E(Q))$ (Proposition 3.4(b) again), and so $\varepsilon_{Q, Q}\left(N_{S}(Q)\right.$ ) is a Sylow $p$-subgroup of $E(Q) \cdot \varepsilon_{Q, Q}\left(N_{S}(Q)\right)$. Thus there is $\chi \in E(Q)$ such that $(\chi \circ \varphi)\left(\varepsilon_{P, P}\left(N_{f}\right)\right)(\chi \circ \varphi)^{-1}$ is contained in $\varepsilon_{Q, Q}\left(N_{S}(Q)\right)$. So by axiom (II) for $\mathcal{T}, \chi \circ \varphi$ extends to a $\mathcal{T}$-morphism defined on $N_{f}, \rho(\chi \circ \varphi)=\rho(\varphi)=f$, and hence $f$ extends to an $\mathcal{F}$-morphism defined on $N_{f}$. Thus $\mathcal{F}$ satisfies axiom (II) for the subgroup $P$.

We have now shown that $\mathcal{F}$ is $\operatorname{Ob}(\mathcal{T})$-saturated. The last two statements $(\mathcal{F}$ is saturated under additional hypotheses) follow from Theorem 1.5(b).

For any transporter system $(S, \mathcal{F}, \mathcal{T})$, we let $\mathcal{T}^{c} \subseteq \mathcal{T}$ denote the full subcategory whose objects are those $P \in \operatorname{Ob}(\mathcal{T})$ which are $\mathcal{F}$-centric. We want to show that $\mathcal{T}$ determines a unique linking system $\mathcal{L}$ associated to $\mathcal{F}$ with object set $\operatorname{Ob}\left(\mathcal{T}^{c}\right)$. When doing this, we use the term "linking system" associated to $\mathcal{F}$ in a slightly more general way than previously: to refer to any category $\mathcal{L}$ which satisfies all of the conditions in Definition 1.6, except that $\operatorname{Ob}(\mathcal{L})$ need not contain all $\mathcal{F}$-centric subgroups.

Proposition 3.7. Let $(S, \mathcal{F}, \mathcal{T})$ be a transporter system. Let $E(P)$, for $P \in \mathrm{Ob}(\mathcal{T})$, be as in Definition 3.1. Then for every $\mathcal{F}$-centric subgroup $P \leq S, E(P)=E_{0}(P) \times Z(P)$, where $E_{0}(P)$ is the subgroup generated by all elements in $E(P)$ of order prime to $p$. We can thus define a centric linking system $\mathcal{L}$ associated to $\mathcal{F}$ by setting $\mathrm{Ob}(\mathcal{L})=\mathrm{Ob}\left(\mathcal{T}^{c}\right)$, and by setting

$$
\operatorname{Mor}_{\mathcal{L}}(P, Q)=\operatorname{Mor}_{\mathcal{T}}(P, Q) / E_{0}(P)
$$

for all $P, Q \in \mathrm{Ob}(\mathcal{L})$.

Proof. By axiom $(\mathrm{C})$, for all $P \in \mathrm{Ob}(\mathcal{T}), E(P)$ commutes with $\varepsilon_{P, P}(P)$ in $\operatorname{Aut}_{\mathcal{T}}(P)$. Hence if $P$ is $\mathcal{F}$-centric, then $\varepsilon_{P, P}(Z(P))$ is central in $E(P)$, and is a Sylow $p$-subgroup by Proposition 3.4. This implies that $E(P)$ splits as a product $Z(P) \times E_{0}(P)$, where $E_{0}(P)$ consists of all elements in $E(P)$ of order prime to $p$.

It is now straightforward to check that $\mathcal{L}$, when defined as above, is a quotient category of $\mathcal{T}^{c}$ (i.e., composition is well defined). Also, axioms (A), (B), and (C) for a transporter system imply that $\mathcal{L}$ satisfies the corresponding axioms for a linking system, and thus is a linking system associated to $\mathcal{F}$.

We have already shown that every morphism in a transporter system is a monomorphism in the categorical sense. We now show that every morphism is also an epimorphism. 
Lemma 3.8. Let $(S, \mathcal{F}, \mathcal{T})$ be a transporter system. Fix subgroups $P, Q, R \in \mathcal{T}$, together with morphisms $\psi \in \operatorname{Mor}_{\mathcal{T}}(P, Q)$ and $\varphi_{1}, \varphi_{2} \in \operatorname{Mor}_{\mathcal{T}}(Q, R)$ such that $\varphi_{1} \circ \psi=$ $\varphi_{2} \circ \psi$. Then $\varphi_{1}=\varphi_{2}$. In other words, all morphisms in $\mathcal{T}$ are epimorphisms in the categorical sense.

Proof. Since $\psi$ is the composite of an isomorphism followed by an inclusion (Lemma $3.2(\mathrm{c}))$, it suffices to prove this when $P \leq Q$ and $\psi=\iota_{P, Q}$ is the inclusion. Also, it suffices to do this when $P \triangleleft Q$ : otherwise it can be shown in several steps using a chain of subgroups linking $P$ to $Q$, each normal in the following one.

Set $P^{\prime}=\rho\left(\varphi_{1}\right)(P)$. By Lemma 3.2(c), $\varphi_{1} \circ \iota_{P, Q}=\varphi_{2} \circ \iota_{P, Q}$ has a unique restriction $\beta=\left.\varphi_{1}\right|_{P, P^{\prime}}=\left.\varphi_{2}\right|_{P, P^{\prime}} \in \operatorname{Iso}_{\mathcal{T}}\left(P, P^{\prime}\right)$. Fix $x \in Q$, set $y_{i}=\rho\left(\varphi_{i}\right)(x)$, and consider the following two squares:
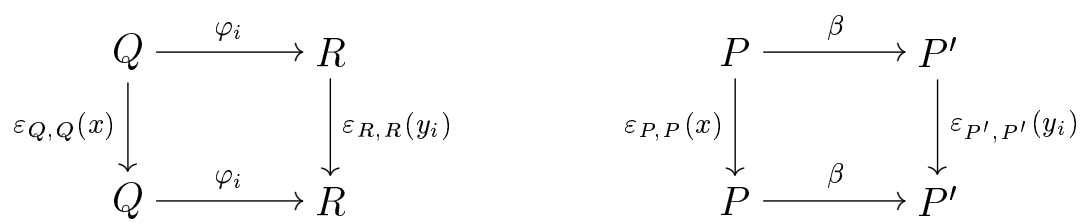

The first square commutes by axiom (C), and the second square is defined to be a restriction of the first. Note that $\varepsilon_{P, P}(x)$ is the restriction of $\varepsilon_{Q, Q}(x)$ since $\varepsilon$ is a functor (and since $\iota_{A, B}=\varepsilon_{A, B}(1)$ for all $A \leq B \leq S$ ). Hence the second square commutes by the uniqueness of restriction morphisms (Lemma 3.2(c)). Thus $\varepsilon_{P^{\prime}, P^{\prime}}\left(y_{1}\right)=\varepsilon_{P^{\prime}, P^{\prime}}\left(y_{2}\right)=$ $\beta \circ \varepsilon_{P, P}(x) \circ \beta^{-1}$. Since $\varepsilon_{P^{\prime}, P^{\prime}}$ is injective, this shows that $y_{1}=y_{2}$. Since this holds for all $x \in Q, \rho\left(\varphi_{1}\right)=\rho\left(\varphi_{2}\right)$.

By axiom (A2), we now get $\varphi_{2}=\varphi_{1} \circ \alpha$ for some $\alpha \in E(Q)$. Hence

$$
\varphi_{1} \circ \alpha \circ \iota_{P, Q}=\varphi_{2} \circ \iota_{P, Q}=\varphi_{1} \circ \iota_{P, Q},
$$

so $\alpha \circ \iota_{P, Q}=\iota_{P, Q}$ by Lemma 3.2 again, and $\alpha=\operatorname{Id}_{Q}$ since $E(Q)$ acts freely on $\operatorname{Mor}_{\mathcal{T}}(P, Q)$ (axiom (A2)). It follows that $\varphi_{1}=\varphi_{2}$.

By analogy with the definition of radical and normal subgroups in a fusion system, we define:

Definition 3.9. Let $(S, \mathcal{F}, \mathcal{T})$ be a transporter system.

- A subgroup $Q \in \mathrm{Ob}(\mathcal{T})$ is called $\mathcal{T}$-radical if $\varepsilon_{Q, Q}(Q)=O_{p}\left(\operatorname{Aut}_{\mathcal{T}}(Q)\right)$.

- An arbitary subgroup $Q \leq S$ (not necessarily an object in $\mathcal{T}$ ) is called normal in $\mathcal{T}$, denoted $Q \triangleleft \mathcal{T}$, if for every morphism $\varphi \in \operatorname{Mor}_{\mathcal{T}}\left(P, P^{\prime}\right)$ in $\mathcal{T}$, there is a morphism $\bar{\varphi} \in \operatorname{Mor}_{\mathcal{T}}\left(P Q, P^{\prime} Q\right)$ such that $\iota_{P^{\prime}, P^{\prime} Q} \circ \varphi=\bar{\varphi} \circ \iota_{P, P Q}$ and $\rho(\bar{\varphi})(Q)=Q$.

If $\mathcal{T}$ is a full subcategory of $\mathcal{T}_{S}(G)$ for some finite group $G$ and $S \in \operatorname{Syl}_{p}(G)$, then for $Q \in \mathrm{Ob}(\mathcal{T}), Q$ is $\mathcal{T}$-radical if and only if $Q$ is a radical $p$-subgroup of $G$ in the usual sense. If $(S, \mathcal{F}, \mathcal{L})$ is a $p$-local finite group and we regard $\mathcal{L}$ as a transporter system, then $P \in \mathrm{Ob}(\mathcal{L})$ is $\mathcal{L}$-radical if and only if it is $\mathcal{F}$-centric and $\mathcal{F}$-radical. More generally, if $(S, \mathcal{F}, \mathcal{T})$ is an abstract transporter system and $P \leq S$ is $\mathcal{F}$-centric and $\mathcal{F}$-radical, then it is not hard to see that $P$ is also $\mathcal{T}$-radical, but not conversely. For example, assume $G=H \rtimes S$ where $H$ has order prime to $p, S \in \operatorname{Syl}_{p}(G)$, and $C_{G}(H) \leq H$. Set $\mathcal{F}=\mathcal{F}_{S}(G)$ and $\mathcal{T}=\mathcal{T}_{S}(G)$. Then every subgroup of $S$ is $\mathcal{T}$-radical, but no proper subgroup of $S$ is $\mathcal{F}$-radical (nor $\mathcal{F}$-centric if $S$ is abelian).

The following proposition is the version for transporter systems of Alperin's fusion theorem. 
Proposition 3.10. Let $(S, \mathcal{F}, \mathcal{T})$ be a transporter system. For each $P, P^{\prime} \in \operatorname{Ob}(\mathcal{T})$ and each $\varphi \in \operatorname{Iso}_{\mathcal{T}}\left(P, P^{\prime}\right)$, there are subgroups

$$
P=P_{0}, P_{1}, \ldots, P_{k}=P^{\prime} \quad \text { and } \quad Q_{i} \geq\left\langle P_{i-1}, P_{i}\right\rangle \quad(i=1, \ldots, k)
$$

where each $Q_{i}$ is $\mathcal{T}$-radical and fully normalized in $\mathcal{F}$, and also automorphisms $\alpha_{i} \in$ $\operatorname{Aut}_{\mathcal{T}}\left(Q_{i}\right)$ and isomorphisms $\varphi_{i} \in \operatorname{Iso}_{\mathcal{T}}\left(P_{i-1}, P_{i}\right)$, such that $\varphi_{i}=\left.\alpha_{i}\right|_{P_{i-1}, P_{i}}$ for each $i$, and $\varphi=\varphi_{k} \circ \cdots \circ \varphi_{1}$.

Proof. Fix $\varphi$, and assume inductively that the result holds for all isomorphisms between larger subgroups of $S$. If $P=P^{\prime}=S$, there is nothing to prove, so we assume that $P, P^{\prime} \supsetneqq S$. Choose a fully normalized subgroup $Q$ in the $\mathcal{F}$-conjugacy class of $P$ and $P^{\prime}$. Since $\varepsilon_{Q, Q}\left(N_{S}(Q)\right)$ is a Sylow $p$-subgroup in $\operatorname{Aut}_{\mathcal{T}}(Q)$ by axiom $\left(\mathrm{I}^{\prime}\right)$, there are isomorphisms $\psi \in \operatorname{Iso}_{\mathcal{T}}(P, Q)$ and $\psi^{\prime} \in \operatorname{Iso}_{\mathcal{T}}\left(P^{\prime}, Q\right)$ such that $\psi \circ \varepsilon_{P, P}\left(N_{S}(P)\right) \circ \psi^{-1} \leq$ $\varepsilon_{Q, Q}\left(N_{S}(Q)\right)$ and similarly for $\psi^{\prime}$. So by axiom (II), $\psi$ extends to a morphism from $N_{S}(P)$ to $N_{S}(Q)$, and similarly for $\psi^{\prime}$, and so the proposition holds for $\psi$ and $\psi^{\prime}$ both. Thus to prove the proposition for $\varphi$, it suffices to prove it for $\psi^{\prime} \circ \varphi \circ \psi^{-1} \in \operatorname{Aut}_{\mathcal{T}}(Q)$. In other words, we are reduced to the case where $P=P^{\prime}$ is fully normalized.

If $P$ is $\mathcal{T}$-radical, then we are done. Otherwise, let $R \supsetneqq P$ be such that $\varepsilon_{P, P}(R)=$ $O_{p}\left(\operatorname{Aut}_{\mathcal{T}}(P)\right)$. By axiom (II) again, any $\varphi \in \operatorname{Aut}_{\mathcal{T}}(P)$ extends to an automorphism of $R$, and again we are done by the induction hypothesis.

If $(S, \mathcal{F}, \mathcal{T})$ is a transporter system and $A \triangleleft \mathcal{T}$, then we define the quotient category $\mathcal{T} / A$ by letting $\operatorname{Ob}(\mathcal{T} / A)$ be the set of all $P / A$ for $A \leq P \in \mathrm{Ob}(\mathcal{T})$, and setting

$$
\operatorname{Mor}_{\mathcal{T} / A}(P / A, Q / A)=\operatorname{Mor}_{\mathcal{T}}(P, Q) / \varepsilon_{P, P}(A)=\varepsilon_{Q, Q}(A) \backslash \operatorname{Mor}_{\mathcal{T}}(P, Q) .
$$

The equivalence between these two formulas for $\operatorname{Mor}_{\mathcal{T} / A}(P / A, Q / A)$ follows from axiom (C). We next show that $\mathcal{T} / A$ is itself a transporter system.

Proposition 3.11. If $(S, \mathcal{F}, \mathcal{T})$ is a p-local finite group, and $A$ is a normal subgroup in $\mathcal{T}$, then $\mathcal{T} / A$ is a transporter system associated to the fusion system $\mathcal{F} / A$.

Proof. We will denote by $[f]$ the morphism in $\operatorname{Mor}_{\mathcal{T} / A}(P / A, Q / A)$ represented by $f$ in $\operatorname{Mor}_{\mathcal{T}}(P, Q)$, and by $f / A$ the morphism in $\operatorname{Mor}_{\mathcal{F} / A}(P / A, Q / A)$ induced by $f$ in $\operatorname{Mor}_{\mathcal{F}}(P, Q)$. So all morphisms in $\mathcal{T} / A$ have the form $[f]$ for some $f \in \operatorname{Mor}(\mathcal{T})$, and all morphisms in $\mathcal{F} / A$ have the form $f / A$ for some $f \in \operatorname{Mor}(\mathcal{F})$.

Let $\bar{\rho}: \mathcal{T} / A \longrightarrow \mathcal{F} / A$ be the functor induced by $\rho: \mathcal{T} \longrightarrow \mathcal{F}$, i.e., $\bar{\rho}([f])=\rho(f) / A$. Let $\bar{\varepsilon}: \mathcal{T}_{\mathrm{Ob}(\mathcal{T} / A)}(S / A) \longrightarrow \mathcal{T} / A$ be the functor which is the identity on objects, and where

$$
\begin{gathered}
\bar{\varepsilon}_{P / A, Q / A}: N_{S / A}(P / A, Q / A) \longrightarrow N_{S}(P, Q) / A \\
=\operatorname{Mor}_{\mathcal{T} / A}(P / A, Q / A) \\
=\operatorname{Mor}_{\mathcal{T}}(P, Q) / A
\end{gathered}
$$

is defined by setting $\bar{\varepsilon}_{P / A, Q / A}(g A)=\left[\varepsilon_{P, Q}(g)\right]$, for all $g \in N_{S}(P, Q)$.

Axiom (A1) holds by definition of $\bar{\rho}$.

Axiom (A2): $\quad E(P / A)$ acts freely on $\operatorname{Mor}_{\mathcal{T} / A}(P / A, Q / A)$. Assume $\varphi \in \operatorname{Mor}_{\mathcal{T}}(P, Q)$ and $\chi \in \operatorname{Aut}_{\mathcal{T}}(P)$ are such that $[\varphi]_{\circ}[\chi]=[\varphi]$ and $[\chi] \in E(P / A)$. Then $\varphi \circ \chi=\varphi \circ \varepsilon_{P, P}(a)$ for some $a \in A, \chi=\varepsilon_{P, P}(a)$ by Lemma 3.2(b), and hence $[\chi]$ is the identity in $E(P / A)$.

$\bar{\rho}_{P, Q}$ is the orbit map for the $E(P)$-action on $\operatorname{Mor}_{\mathcal{T} / A}(P / A, Q / A)$. Fix $P$ and $Q$, and $\varphi, \psi \in \operatorname{Mor}_{\mathcal{T}}(P, Q)$. If $[\psi]=[\varphi] \circ[\chi]$ for some $[\chi] \in E(P / A)$, then clearly $\bar{\rho}([\psi])=\bar{\rho}([\varphi])$. Conversely, assume that $\bar{\rho}([\varphi])=\bar{\rho}([\psi])$; we must show that they are in the same $E(P / A)$-orbit. We have

$$
\rho(\varphi)(P) / A=\operatorname{Im}(\bar{\rho}([\varphi]))=\operatorname{Im}(\bar{\rho}([\psi]))=\rho(\psi)(P) / A,
$$


and so $\rho(\varphi)(P)=\rho(\psi)(P)$. Since every morphism in $\mathcal{F}$ factors as an $\mathcal{F}$-isomorphism followed by an inclusion, this implies that there is $\alpha \in \operatorname{Aut}_{\mathcal{F}}(P)$ such that $\rho(\psi)=$ $\rho(\varphi) \circ \alpha$. So by Lemma 3.2(a), there is $\chi \in \operatorname{Aut}_{\mathcal{T}}(P)$ such that $\psi=\varphi \circ \chi$ and $\rho(\chi)=\alpha$. Then $\bar{\rho}([\chi])=\alpha / A=\operatorname{Id}_{P / A}$, and hence $[\chi] \in E(P / A)$.

$E(Q)$ acts freely on $\operatorname{Mor}_{\mathcal{T}}(P, Q)$. Assume $\varphi \in \operatorname{Mor}_{\mathcal{T}}(P, Q)$ and $\chi \in \operatorname{Aut}_{\mathcal{T}}(Q)$ are such that $[\chi] \circ[\varphi]=[\varphi]$ and $[\chi] \in E(Q / A)$. Then for some $a \in A$,

$$
\chi \circ \varphi=\varphi \circ \varepsilon_{P, P}(a)=\varepsilon_{Q, Q}(\rho(\varphi)(a)) \circ \varphi
$$

where the second equality holds by axiom (C) for a transporter system; $\rho(\varphi)(a) \in A$ since $A$ is normal in $\mathcal{F}$; $\chi=\varepsilon_{Q, Q}(\rho(\varphi)(a))$ by Lemma 3.8; and hence $[\chi]$ is the identity in $E(Q / A)$.

Axiom (B) for $\mathcal{T} / A$ follows directly from axiom (B) for $\mathcal{T}$.

Axiom (C) for $\mathcal{T} / A$ is a consequence of axiom (C) applied to $\mathcal{T}$. More precisely, for each $f \in \operatorname{Mor}_{\mathcal{T}}(P, Q)$ and each $g \in P$,

$$
\begin{aligned}
{[f] \circ \bar{\varepsilon}_{P / A, P / A}(g A) } & =[f] \circ\left[\varepsilon_{P, P}(g)\right]=\left[f \circ \varepsilon_{P, P}(g)\right]=\left[\varepsilon_{Q, Q}(\rho(f)(g)) \circ f\right] \\
& =\bar{\varepsilon}_{Q / A, Q / A}(\rho(f)(g) A) \circ[f]=\bar{\varepsilon}_{Q / A, Q / A}(\bar{\rho}([f])(g A)) \circ[f] .
\end{aligned}
$$

Axiom (I): By axiom (I) for $\mathcal{T}, \varepsilon_{S, S}(S)$ is a Sylow $p$-subgroup of $\operatorname{Aut}_{\mathcal{T}}(S)$. So upon diving out by $A$, we get that $\bar{\varepsilon}_{S / A, S / A}(S / A)$ is a Sylow $p$-subgoup of $\operatorname{Aut}_{\mathcal{T} / A}(S / A)$.

Axiom (II): Fix $[f] \in \operatorname{Iso}_{\mathcal{T} / A}(P / A, Q / A)$, and let $P / A \triangleleft \bar{P} / A \leq S / A$ and $Q / A \triangleleft$ $\bar{Q} / A \leq S / A$ be such that

$$
[f] \circ \bar{\varepsilon}_{P / A, P / A}(\bar{P} / A) \circ[f]^{-1} \leq \bar{\varepsilon}_{Q / A, Q / A}(\bar{Q} / A) .
$$

Then for any lifting of $[f]$ to $f \in \operatorname{Iso}_{\mathcal{T}}(P, Q)$,

$$
f \circ \varepsilon_{P, P}(\bar{P}) \circ f^{-1} \leq \varepsilon_{Q, Q}(\bar{Q}) .
$$

So $f$ extends to some $\bar{f} \in \operatorname{Mor}_{\mathcal{T}}(\bar{P}, \bar{Q})$ by axiom (II) applied to $\mathcal{T}$, and thus $[f]$ extends to $[\bar{f}]$ in $\operatorname{Mor}_{\mathcal{T} / A}(\bar{P} / A, \bar{Q} / A)$.

The next proposition shows that a transporter system over $S$ which contains all subgroups of $S$ as objects is the transporter category of a finite group.

Proposition 3.12. Let $\mathcal{T}$ be a transporter system over a p-group $S$ for which $\mathrm{Ob}(\mathcal{T})$ is the set of all subgroups of $S$. Set $G=\operatorname{Aut}_{\mathcal{T}}(1)$, and identify $S$ as a subgroup of $G$ via $\varepsilon_{1,1}$. Then $S \in \operatorname{Syl}_{p}(G)$, and there is an isomorphism of 4-tuples

$$
(\mathcal{T}, \mathcal{F}, \rho, \varepsilon) \cong\left(\mathcal{T}_{S}(G), \mathcal{F}_{S}(G),\left(g \mapsto c_{g}\right), \text { incl }\right)
$$

Proof. Note first that $S \in \operatorname{Syl}_{p}(G)$ by axiom (I') (Proposition 3.4), applied with $P=1$. By (II) (applied with $P=Q=1$ ), for each $P, Q \leq S$ and each $g \in N_{G}(P, Q)$, there is some $\widehat{\varepsilon}_{P, Q}(g) \in \operatorname{Mor}_{\mathcal{T}}(P, Q)$ which extends $g \in \operatorname{Aut}_{\mathcal{T}}(1)$, and this extension is unique by Lemma 3.8. If $R$ is another subgroup and $h \in N_{G}(Q, R)$, then $\widehat{\varepsilon}_{P, R}(h g)=$ $\widehat{\varepsilon}_{Q, R}(h) \circ \widehat{\varepsilon}_{P, Q}(g)$ by the uniqueness of the construction. 
We have thus defined a functor $\widehat{\varepsilon}$ from $\mathcal{T}_{S}(G)$ to $\mathcal{T}$ which is the identity on objects. Consider the following diagram:

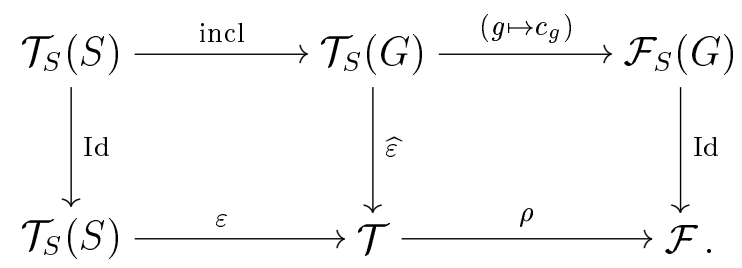

By Lemma 3.3 (applied with $P=Q=1$ ), for $g \in N_{G}(P, Q), \rho\left(\widehat{\varepsilon}_{P, Q}(g)\right)$ is conjugation by $g$. This proves that $\mathcal{F}_{S}(G) \subseteq \mathcal{F}$, and that the right hand square in the diagram commutes. For $g \in N_{S}(P, Q), \varepsilon_{P, Q}(g)$ is an extension of $\varepsilon_{1,1}(g)$ (since $\varepsilon$ is a functor). So $\varepsilon$ is the restriction of $\widehat{\varepsilon}$ to $\mathcal{T}_{S}(S)$, and thus the left hand square commutes.

It remains to show that $\widehat{\varepsilon}$ is an isomorphism of categories; it then follows from the surjectivity of $\rho$ that $\mathcal{F}_{S}(G)=\mathcal{F}$. For all $P, Q \leq S$ and $\varphi \in \operatorname{Mor}_{\mathcal{T}}(P, Q)$, there is a uniquely defined restriction $\left.\varphi\right|_{1,1} \in \operatorname{Aut}_{\mathcal{T}}(1)=G$ by Lemma 3.2(c). Set $g=\left.\varphi\right|_{1,1}$ for short. By Lemma 3.3 again, $g x g^{-1}=\rho(\varphi)(x) \in Q$ for all $x \in P$ (recall that we identify $S$ as a subgroup of $G$ via $\left.\varepsilon_{1,1}\right)$, and thus $g \in N_{G}(P, Q)$. It follows that $\varphi=\widehat{\varepsilon}_{P, Q}(g) ; g$ is unique by the uniqueness of the restriction; and thus $\widehat{\varepsilon}_{P, Q}$ is a bijection.

More generally, if $(S, \mathcal{F}, \mathcal{T})$ is a transporter system, and there is some $Q \in \operatorname{Ob}(\mathcal{T})$ such that $Q \triangleleft \mathcal{T}$, then one can show that $\mathcal{T}$ is isomorphic to a full subcategory of $\mathcal{T}_{S}\left(\operatorname{Aut}_{\mathcal{T}}(Q)\right)$

We next describe another way to construct new transporter systems as quotients of other transporter systems; a construction which will be useful in Section 6 . As in Definition 3.1, for a transporter system $(S, \mathcal{F}, \mathcal{T})$, we let $E(P)$ be the kernel

$$
E(P)=\operatorname{Ker}\left[\operatorname{Aut}_{\mathcal{T}}(P) \longrightarrow \operatorname{Aut}_{\mathcal{F}}(P)\right]
$$

for all $P \in \mathrm{Ob}(\mathcal{T})$. We regard $E$ as a functor $E: \mathcal{T}^{\mathrm{op}} \longrightarrow$ Gps: for each $\varphi \in$ $\operatorname{Mor}_{\mathcal{T}}(P, Q)$ and each $x \in E(Q), E(\varphi)(x) \in E(P)$ is the unique morphism such that

$$
\varphi \circ E(\varphi)(x)=x \circ \varphi \in \operatorname{Mor}_{\mathcal{T}}(P, Q) .
$$

Proposition 3.13. Fix a transporter system $(S, \mathcal{F}, \mathcal{T})$. Let $E_{0}: \mathcal{T}^{\mathrm{op}} \longrightarrow \mathrm{Gps}$ be a subfunctor of $E$ which satisfies the following two conditions for each $P, Q \in \mathrm{Ob}(\mathcal{T})$ and each $\varphi \in \operatorname{Mor}_{\mathcal{T}}(P, Q)$ :

(a) $E_{0}(P)$ has order prime to $p$, and

(b) $E_{0}(Q)=E(\varphi)^{-1}\left(E_{0}(P)\right)$.

Let $\mathcal{T} / E_{0}$ be the quotient category which has the same objects as $\mathcal{T}$, and where

$$
\operatorname{Mor}_{\mathcal{T} / E_{0}}(P, Q)=\operatorname{Mor}_{\mathcal{T}}(P, Q) / E_{0}(P) .
$$

Then $\mathcal{T} / E_{0}$ is a transporter system associated to $\mathcal{F}$.

Proof. The assumption that $E_{0}$ is a subfunctor implies that $\mathcal{T} / E_{0}$ is a category (i.e., that composition is well defined). The structure functors

$$
\mathcal{T}_{\mathrm{Ob}(\mathcal{T})}(S) \stackrel{\varepsilon^{\prime}}{\longrightarrow} \mathcal{T} / E_{0} \stackrel{\rho^{\prime}}{\longrightarrow} \mathcal{F}
$$

are defined in the obvious way. Most of the axioms in Definition 3.1 are easily carried over from $\mathcal{T}$ to $\mathcal{T} / E_{0}$. The injectivity of $\varepsilon^{\prime}$, and axiom $(\mathrm{I})\left(\varepsilon_{S, S}^{\prime}(S) \in \operatorname{Syl}_{p}\left(\operatorname{Aut}_{\mathcal{T} / E_{0}}(S)\right)\right)$ follow from (a). The freeness of the action of $E(Q) / E_{0}(Q)$ on $\operatorname{Mor}_{\mathcal{T}}(P, Q) / E_{0}(P)$ (part of axiom (A2)) follows from (b). 
The only axiom which requires a little more explanation is (II). Fix $\varphi^{\prime}=[\varphi]$ in $\operatorname{Iso}_{\mathcal{T} / E_{0}}(P, Q)$ (where $\left.\varphi \in \operatorname{Iso}_{\mathcal{T}}(P, Q)\right)$. Assume $\bar{P}$ and $\bar{Q}$ are such that $P \triangleleft \bar{P}, Q \triangleleft \bar{Q}$, and

$$
\varphi^{\prime} \circ \varepsilon_{P, P}^{\prime}(\bar{P}) \circ \varphi^{\prime-1} \leq \varepsilon_{Q, Q}^{\prime}(\bar{Q}) \leq \operatorname{Aut}_{\mathcal{T} / E_{0}}(Q) .
$$

Equivalently, in $\mathcal{T}$, this means that

$$
\varphi \circ \varepsilon_{P, P}(\bar{P}) \circ \varphi^{-1} \leq \varepsilon_{Q, Q}(\bar{Q}) \cdot E_{0}(Q) .
$$

Since $\left|E_{0}(Q)\right|$ is prime to $p, \varepsilon_{Q, Q}(\bar{Q})$ is a Sylow $p$-subgroup of $\varepsilon_{Q, Q}(\bar{Q}) \cdot E_{0}(Q)$. Hence there is $\alpha \in E_{0}(Q)$ such that $(\alpha \varphi) \circ \varepsilon_{P, P}(\bar{P}) \circ(\alpha \varphi)^{-1} \leq \varepsilon_{Q, Q}(\bar{Q})$. Thus $\alpha \varphi$ can be extended to some $\bar{\varphi} \in \operatorname{Mor}_{\mathcal{T}}(\bar{P}, \bar{Q})$ by axiom (II) for $\mathcal{T}$, and so $\varphi^{\prime}=[\varphi]=[\alpha \varphi]$ extends to the morphism $\bar{\varphi}^{\prime}=[\bar{\varphi}] \in \operatorname{Mor}_{\mathcal{T} / E_{0}}(\bar{P}, \bar{Q})$.

The following is an example of how Proposition 3.13 will be applied in Section 6. Fix a finite group $G$, a normal subgroup $H \triangleleft G$, and a $\operatorname{Sylow} \operatorname{subgroup} S \in \operatorname{Syl}_{p}(G)$. Let $\mathcal{H}$ be the set of all subgroups $P \leq S$ such that $(Z(P) \cap H) \in \operatorname{Syl}_{p}\left(C_{H}(P)\right)$. For each $P \in \mathcal{H}, C_{H}(P)=(Z(P) \cap H) \times C_{H}^{\prime}(P)$, where $C_{H}^{\prime}(P)$ is the set (a subgroup) of all elements of $C_{H}(P)$ of order prime to $p$. Then $C_{H}^{\prime}$ is a subfunctor of $C_{G}$ which satisfies conditions (a) and (b) in Proposition 3.13, and hence $\mathcal{T}_{\mathcal{H}}(G) / C_{H}^{\prime}$ is a transporter system associated to $\mathcal{F}_{S}(G)$. When $H=G$, then this is of course just the centric linking system $\mathcal{L}_{S}^{c}(G)$.

The next proposition describes the opposite of this construction: it describes general conditions for an extension of a transporter system to again be a transporter system associated to the same fusion system. However, its main purpose is to show how to construct any centric transporter system associated to a given $\mathcal{F}$ - any transporter system whose objects are the $\mathcal{F}$-centric subgroups - as an extension of a linking system associated to $\mathcal{F}$.

We refer to Definition A.5 for the definition of a source regular extension of a category. For any source regular extension $\mathcal{T}^{\prime} \stackrel{\nu}{\longrightarrow} \mathcal{T}$ of $\mathcal{T}$, we let $\widetilde{K}: \mathcal{T}^{\text {op }} \longrightarrow$ Gps denote the "kernel functor", defined on objects by setting $\widetilde{K}(P)=\operatorname{Ker}\left[\operatorname{Aut}_{\mathcal{T}^{\prime}}(P) \longrightarrow \operatorname{Aut}_{\mathcal{T}}(P)\right]$, and on morphisms by sending $\varphi \in \operatorname{Mor}_{\mathcal{T}^{\prime}}(P, Q)$ to the unique homomorphism $\widetilde{K}(\varphi) \in$ $\operatorname{Hom}(\widetilde{K}(Q), \widetilde{K}(P))$ such that $\alpha \circ \varphi=\varphi \circ \widetilde{K}(\varphi)(\alpha)$ for all $\alpha \in \widetilde{K}(Q)$. (Note that $\widetilde{K}$ as defined here is a functor from $\mathcal{T}^{\prime}$ to groups, as opposed to the functor $K^{*}$ from $\mathcal{T}$ to groups up to conjugacy defined in Lemma A.7(c).)

Proposition 3.14. Fix a transporter system $(S, \mathcal{F}, \mathcal{T})$ and a source regular extension $\mathcal{T}^{\prime} \stackrel{\nu}{\longrightarrow} \mathcal{T}$ of $\mathcal{T}$. Let $\widetilde{K}: \mathcal{T}^{\mathrm{op}} \longrightarrow$ Gps be the kernel functor. Then $\mathcal{T}^{\prime}$ can be given a structure of a transporter system associated to $\mathcal{F}$ if the following conditions hold for all $P \in \mathrm{Ob}\left(\mathcal{T}^{\prime}\right)=\mathrm{Ob}(\mathcal{T}):$

(a) $\widetilde{K}(P)$ is finite of order prime to $p$.

(b) $\widetilde{K}(\varphi)$ is a monomorphism for all $\varphi \in \operatorname{Mor}(\mathcal{T})$.

(c) For all $g \in P, \widetilde{K}\left(\varepsilon_{P, P}(g)\right)=\operatorname{Id}_{\widetilde{K}(P)}$.

(d) If $P \leq \bar{P} \leq N_{S}(P)$ and $x \in \widetilde{K}(P)$ are such that $\widetilde{K}\left(\delta_{P, P}(g)\right)(x)=x$ for all $g \in \bar{P}$, then $x \in \operatorname{Im}\left(\widetilde{K}\left(\iota_{P, \bar{P}}\right)\right)$.

Proof. Let $\mathcal{T}_{\mathrm{Ob}(\mathcal{T})}(S) \stackrel{\varepsilon}{\longrightarrow} \mathcal{T} \stackrel{\rho}{\longrightarrow} \mathcal{F}$ be the structure functors for $\mathcal{T}$, and set $\rho^{\prime}=\rho \circ \nu$. By (c) (applied with $P=S$ ), there is a unique homomorphism $\varepsilon_{S, S}^{\prime}: S \longrightarrow \operatorname{Aut}_{\mathcal{T}^{\prime}}(S)$ 
such that $\nu_{S, S} \circ \varepsilon_{S, S}^{\prime}=\varepsilon_{S, S}$. For each $P \leq S$, choose some $\varepsilon_{P, S}^{\prime}(1) \in \operatorname{Mor}_{\mathcal{T}^{\prime}}(P, S)$ such that $\nu_{P, S} \circ \varepsilon_{P, S}^{\prime}(1)=\varepsilon_{P, S}(1)$. By Lemma A.7(a), for each $P, Q \in \mathrm{Ob}\left(\mathcal{T}^{\prime}\right)$ and each $g \in N_{S}(P, Q)$, there is a unique morphism $\varepsilon_{P, Q}^{\prime}(g) \in \operatorname{Mor}_{\mathcal{T}^{\prime}}(P, Q)$ such that

$$
\varepsilon_{Q, S}^{\prime}(1) \circ \varepsilon_{P, Q}^{\prime}(g)=\varepsilon_{S, S}^{\prime}(g) \circ \varepsilon_{P, S}^{\prime}(1),
$$

and this defines a functor $\varepsilon^{\prime}$ such that $\nu \circ \varepsilon^{\prime}=\varepsilon$.

Axiom (A1) for $\mathcal{T}^{\prime}$ follows immediately, (A2) by (b) and since $\nu$ is source regular, (B) since $\rho^{\prime} \circ \varepsilon^{\prime}=\rho \circ \varepsilon$, and (I) from (a) and the corresponding axiom for the transporter system $\mathcal{T}$ ).

We next check axiom (C). Fix $\varphi \in \operatorname{Mor}_{\mathcal{T}^{\prime}}(P, Q)$ and $g \in P$, and set $h=\rho^{\prime}(\varphi)(g)$ for short. By Lemma A.7(a), there is a unique automorphism $\gamma \in \operatorname{Aut}_{\mathcal{T}^{\prime}}(P)$ such that $\nu_{P, P}(\gamma)=\varepsilon_{P, P}(g)$, and such that the following square commutes in $\mathcal{T}^{\prime}$ :

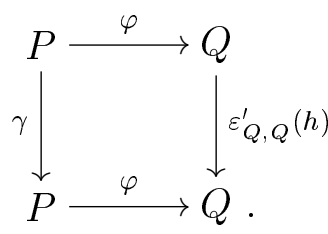

Set $|h|=p^{k}$. By juxtaposing copies of the above square (and by the uniqueness in Lemma A.7(a)), $\gamma^{p^{k}}=$ Id. By $(\mathrm{c}), \nu_{P, P}^{-1}\left(\varepsilon_{P, P}(P)\right)$ is the product of $\widetilde{K}(P)$ with a $p$ group, hence $\varepsilon_{P, P}(g)$ has a unique lifting to $\operatorname{Aut}_{\mathcal{T}^{\prime}}(P)$ of $p$-power order, and this proves that $\gamma=\varepsilon_{P, P}^{\prime}(g)$.

It remains to prove axiom (II) for $\mathcal{T}^{\prime}$. Fix $P \triangleleft \bar{P} \leq S, Q \triangleleft \bar{Q} \leq S$, and $\varphi \in$ $\operatorname{Iso}_{\mathcal{T}^{\prime}}(P, Q)$ such that $\varphi \varepsilon_{P, P}^{\prime}(\bar{P}) \varphi^{-1} \leq \varepsilon_{Q, Q}^{\prime}(\bar{Q})$. Then $\nu(\varphi)$ extends to a morphism on $\bar{P}$ by axiom (II) for $\mathcal{T}$, and hence there is $\psi \in \operatorname{Mor}_{\mathcal{T}^{\prime}}(\bar{P}, \bar{Q})$ such that $\nu\left(\left.\psi\right|_{P, Q}\right)=\nu(\varphi)$. Let $\alpha \in \widetilde{K}(P)$ be the unique element such that $\left.\psi\right|_{P, Q}=\varphi \circ \alpha$; then $\alpha$ normalizes $\varepsilon_{P, P}^{\prime}(\bar{P})$. Since $\alpha \in \widetilde{K}(P)$ which is normal in $\operatorname{Aut}_{\mathcal{T}^{\prime}}(P)$, this shows that

$$
\left[\alpha, \varepsilon_{P, P}^{\prime}(\bar{P})\right] \in \widetilde{K}(P) \cap \varepsilon_{P, P}^{\prime}(\bar{P})=1,
$$

where the intersection is trivial since one of the groups has order prime to $p$ and the other is a $p$-group. We can now apply condition (d) to show that $\alpha$ extends to $\bar{\alpha} \in \operatorname{Aut}_{\mathcal{T}^{\prime}}(\bar{P})$, and hence $\psi \circ \bar{\alpha}^{-1}$ is an extension of $\varphi$ to $\bar{P}$.

\section{Homotopy PROperties OF TRANSPORTER SYSTEMS}

We collect here some results about the fundamental group, the homotopy type, and the cohomology of nerves of transporter systems. Several results from the appendix will be needed when proving these.

By Proposition A.3(a), for any transporter system $(S, \mathcal{F}, \mathcal{T}), \pi_{1}(|\mathcal{T}|)$ is the free group on the morphisms in $\mathcal{T}$, modulo relations given by composition, and by setting inclusion morphisms $\iota_{P, Q}$ equal to the identity. More precisely, let

$$
\theta: \operatorname{Mor}(\mathcal{T}) \longrightarrow \pi_{1}(|\mathcal{T}|)
$$

be the map which sends $\varphi \in \operatorname{Mor}_{\mathcal{T}}(P, Q)$ to the loop (based at the vertex $S$ ) formed by the edges $\iota_{Q, S} \cdot \varphi \cdot \iota_{P, S}{ }^{-1}$ (composed from right to left). This clearly sends composites to products and sends inclusions to the identity, and Proposition A.3 says that $\theta$ is universal among all maps defined on $\operatorname{Mor}(\mathcal{T})$ with these properties. 
If $\varphi_{0}, \varphi \in \operatorname{Mor}(\mathcal{T})$ are such that $\varphi_{0}$ is a restriction of $\varphi$, then they differ by composition with inclusion morphisms, and hence $\theta\left(\varphi_{0}\right)=\theta(\varphi)$. Thus by Proposition 3.10 (Alperin's fusion theorem for transporter systems), $\pi_{1}(|\mathcal{T}|)$ is generated by the subgroups $\theta\left(\operatorname{Aut}_{\mathcal{T}}(P)\right)$ for fully normalized $\mathcal{T}$-radical subgroups $P \in \mathrm{Ob}(\mathcal{T})$.

The following proposition describes one way to construct transporter subsystems, as "kernels" of homomorphisms defined on the fundamental group.

Proposition 4.1. Let $(S, \mathcal{F}, \mathcal{T})$ be a transporter system. Fix a finite group $\Gamma$, and a homomorphism $\Phi: \pi_{1}(|\mathcal{T}|) \longrightarrow \Gamma$. We also identify $\Phi$ with $\Phi \circ \theta$; i.e., as a function defined on $\operatorname{Mor}(\mathcal{T})$ which sends composites to products and inclusion morphisms to the identity. For each subgroup $H \leq \Gamma$, set $S_{H}=\left(\Phi \circ \varepsilon_{S, S}\right)^{-1}(H)$, a subgroup of $S$, and assume that $S_{1} \in \mathrm{Ob}(\mathcal{T})$. Let $\mathcal{T}_{H} \subseteq \mathcal{T}$ be the subcategory defined by setting

$$
\operatorname{Ob}\left(\mathcal{T}_{H}\right)=\left\{P \in \operatorname{Ob}(\mathcal{T}) \mid P \leq S_{H}\right\} \quad \text { and } \quad \operatorname{Mor}_{\mathcal{T}_{H}}(P, Q)=\left\{\varphi \in \operatorname{Mor}_{\mathcal{T}}(P, Q) \mid \Phi(\varphi) \in H\right\} .
$$

Let $\mathcal{F}_{H} \subseteq \mathcal{F}$ be the fusion system over $S_{H}$ generated by $\rho\left(\mathcal{T}_{H}\right)$, and let

$$
\mathcal{T}_{\mathrm{Ob}\left(\mathcal{T}_{H}\right)}\left(S_{H}\right) \stackrel{\varepsilon_{H, H}}{\longrightarrow} \mathcal{T}_{H} \stackrel{\rho_{H}}{\longrightarrow} \mathcal{F}_{H}
$$

be the restrictions of $\varepsilon$ and $\rho$. Then the following hold for each $H \leq \Gamma$.

(a) $\Phi(\operatorname{Mor}(\mathcal{T}))=\Phi\left(\operatorname{Aut}_{\mathcal{T}}\left(S_{1}\right)\right)$.

(b) $\mathcal{T}_{H}$ is a transporter system associated to the fusion system $\mathcal{F}_{H}$ if and only if $\varepsilon_{S_{1}, S_{1}}\left(S_{H}\right) \in \operatorname{Syl}_{p}\left(\operatorname{Aut}_{\mathcal{T}_{H}}\left(S_{1}\right)\right)$. In particular, $\mathcal{T}_{H}$ is a transporter system whenever $H \triangleleft \Gamma$.

(c) Assume that $\varepsilon_{S_{1}, S_{1}}\left(S_{H}\right) \in \operatorname{Syl}_{p}\left(\operatorname{Aut}_{\mathcal{T}_{H}}\left(S_{1}\right)\right)$; and also that for all $P \leq S$ fully centralized in $\mathcal{F}, C_{S_{1}}(P) \leq P$ implies $P \in \mathrm{Ob}(\mathcal{T})$ ( $\Phi$ is an admissible homomorphism in the sense of Definition 5.10). Then $\mathcal{F}_{H}$ is a saturated fusion system.

(d) Assume $P \in \mathrm{Ob}(\mathcal{T})$ implies $P \cap S_{1} \in \mathrm{Ob}(\mathcal{T})$. Then $\left|\mathcal{T}_{H}\right|$ has the homotopy type of the covering space of $|\mathcal{T}|$ with fundamental group $\Phi^{-1}(H)$.

Proof. In general, for any $P \leq S$, we write $P_{1}=P \cap S_{1}$ for short. We first claim, for all $P, Q \leq S$ such that $P_{1}, Q_{1} \in \mathrm{Ob}(\mathcal{T})$, that there is a well defined restriction map

$$
r=r_{P, Q}: \operatorname{Mor}_{\mathcal{T}}(P, Q) \longrightarrow \operatorname{Mor}_{\mathcal{T}}\left(P_{1}, Q_{1}\right) .
$$

In other words, for all $\psi \in \operatorname{Mor}_{\mathcal{T}}(P, Q)$,

$$
\iota_{Q_{1}, Q} \circ r(\psi)=\psi \circ \iota_{P_{1}, P} \quad \text { for all } \psi \in \operatorname{Mor}_{\mathcal{T}}(P, Q) .
$$

By Lemma 3.2(c), this means proving that $\rho(\psi)\left(P_{1}\right) \leq Q_{1}$. For all $g \in P_{1}$,

$$
\Phi\left(\varepsilon_{S, S}(\rho(\psi)(g))\right)=\Phi\left(\varepsilon_{Q, Q}(\rho(\psi)(g))\right)=\Phi(\psi) \cdot \Phi\left(\varepsilon_{P, P}(g)\right) \cdot \Phi(\psi)^{-1}=1
$$

by axiom $(\mathrm{C})$ for $\mathcal{T}$, so $\rho(\psi)(g) \in S_{1}$. Thus $\rho(\psi)\left(P_{1}\right) \leq Q_{1}$, and these restriction maps are all defined. Note also that (1) implies $\Phi(r(\psi))=\Phi(\psi)$ for all $\psi$.

(a) By Proposition 3.10 (Alperin's fusion theorem for transporter systems), $\Phi(\operatorname{Mor}(\mathcal{T}))$ is contained in the subgroup generated by all $\Phi\left(\operatorname{Aut}_{\mathcal{T}}(P)\right)$ for fully normalized $P \in$ $\operatorname{Ob}(\mathcal{T})$. So it suffices to show that $\Phi\left(\operatorname{Aut}_{\mathcal{T}}(P)\right) \leq \Phi\left(\operatorname{Aut}_{\mathcal{T}}\left(S_{1}\right)\right)$ for all $P \in \operatorname{Ob}(\mathcal{T})$ fully normalized in $\mathcal{F}$. Fix such a $P$, and fix $\alpha \in \operatorname{Aut}_{\mathcal{T}}(P)$. We can assume inductively that $\Phi\left(\operatorname{Aut}_{\mathcal{T}}(Q)\right) \leq \Phi\left(\operatorname{Aut}_{\mathcal{T}}\left(S_{1}\right)\right)$ for all $Q \leq S$ such that $Q \supsetneqq P$. If $P \geq S_{1}$, then $r_{P, P}(\alpha) \in \operatorname{Aut}_{\mathcal{T}}\left(S_{1}\right)$, and so $\Phi(\alpha)=\Phi(r(\alpha)) \in \Phi\left(\operatorname{Aut}_{\mathcal{T}}\left(S_{1}\right)\right)$.

We are thus reduced to the case where $P \supsetneqq S_{1}$. Then $P \supsetneqq P S_{1}$, so $P \supsetneqq N_{P S_{1}}(P)=$ $P \cdot N_{S_{1}}(P)$. Since $P$ is fully normalized, $\varepsilon_{P, P}\left(N_{S}(P)\right) \in \operatorname{Syl}_{p}\left(\operatorname{Aut}_{\mathcal{T}}(P)\right)$ by axiom $\left(\mathrm{I}^{\prime}\right)$. Set

$$
\operatorname{Aut}_{1}(P)=\operatorname{Ker}\left[\operatorname{Aut}_{\mathcal{T}}(P) \stackrel{\Phi}{\longrightarrow} \Gamma\right]
$$


so that

$$
\varepsilon_{P, P}\left(N_{S_{1}}(P)\right)=\operatorname{Aut}_{1}(P) \cap \varepsilon_{P, P}\left(N_{S}(P)\right) \in \operatorname{Syl}_{p}\left(\operatorname{Aut}_{1}(P)\right) .
$$

Since all Sylow $p$-subgroups of $\operatorname{Aut}_{1}(P)$ are conjugate,

$$
\alpha \varepsilon_{P, P}\left(N_{S_{1}}(P)\right) \alpha^{-1}=\beta \varepsilon_{P, P}\left(N_{S_{1}}(P)\right) \beta^{-1}
$$

for some $\beta \in \operatorname{Aut}_{1}(P)$, and thus $\beta^{-1} \circ \alpha$ normalizes $\varepsilon_{P, P}\left(N_{S_{1}}(P)\right)$. By axiom (II), $\beta^{-1} \circ \alpha$ extends to an automorphism $\bar{\alpha} \in \operatorname{Aut}_{\mathcal{T}}\left(N_{P S_{1}}(P)\right)$; i.e., $\left.\bar{\alpha}\right|_{P, P}=\beta^{-1} \circ \alpha$. Then $\Phi(\bar{\alpha})=\Phi\left(\beta^{-1} \circ \alpha\right)=\Phi(\alpha)$. Since $\Phi(\bar{\alpha}) \in \Phi\left(\operatorname{Aut}_{\mathcal{T}}\left(S_{1}\right)\right)$ by the induction hypothesis, this finishes the proof of (a).

(b) The axioms (A1), (A2), (B), (C), and (II) of a transporter system for $\mathcal{T}_{H}$ follow easily from the corresponding axioms for $\mathcal{T}$, without any restrictions on $H$. Hence $\mathcal{T}_{H}$ is a transporter system if and only if axiom (I) holds.

Set $A=\operatorname{Aut}_{\mathcal{T}}\left(S_{1}\right)$ and $A_{H}=\operatorname{Aut}_{\mathcal{T}_{H}}\left(S_{1}\right)=\{\alpha \in A \mid \Phi(\alpha) \in H\}$ for short. Consider the restriction map

$$
r_{S_{H}, S_{H}}: \operatorname{Aut}_{\mathcal{T}}\left(S_{H}\right) \longrightarrow A=\operatorname{Aut}_{\mathcal{T}}\left(S_{1}\right)
$$

as defined above; an injective map by Lemma 3.8. By axiom (II) (and Lemma 3.3), $\operatorname{Im}\left(r_{S_{H}, S_{H}}\right)$ is the normalizer in $A$ of $\varepsilon_{S_{1}, S_{1}}\left(S_{H}\right)$. Thus

$$
\varepsilon_{S_{H}, S_{H}}\left(S_{H}\right) \in \operatorname{Syl}_{p}\left(\operatorname{Aut}_{\mathcal{T}_{H}}\left(S_{H}\right)\right) \quad \Longleftrightarrow \quad \varepsilon_{S_{1}, S_{1}}\left(S_{H}\right) \in \operatorname{Syl}_{p}\left(N_{A_{H}}\left(\varepsilon_{S_{1}, S_{1}}\left(S_{H}\right)\right)\right) .
$$

Since a proper subgroup of a $p$-group is properly contained in its normalizer, this shows that axiom (I) holds if and only if $\varepsilon_{S_{1}, S_{1}}\left(S_{H}\right) \in \operatorname{Syl}_{p}\left(A_{H}\right)$.

Now, $S_{1}$ is fully normalized in $\mathcal{F}$, since it is the only subgroup in its $\mathcal{F}_{\text {-conjugacy }}$ class. Hence $\varepsilon_{S_{1}, S_{1}}(S) \in \operatorname{Syl}_{p}(A)$ by axiom $\left(\mathrm{I}^{\prime}\right)$. So if $H \triangleleft \Gamma$, then $\varepsilon_{S_{1}, S_{1}}\left(S_{H}\right)$ is a Sylow subgroup of $A_{H}$ by Lemma 1.9(b).

(c) Now assume $\varepsilon_{S_{1}, S_{1}}\left(S_{H}\right) \in \operatorname{Syl}_{p}\left(\operatorname{Aut}_{\mathcal{T}_{H}}\left(S_{1}\right)\right)$. Hence $\mathcal{T}_{H}$ is a transporter system by (b). Assume also that for all $P \leq S$ fully centralized in $\mathcal{F}, C_{S_{1}}(P) \leq P$ implies $P \in \mathrm{Ob}(\mathcal{T})$. In particular, if $P \leq S_{H}$ is $\mathcal{F}_{H^{-}}$centric, then $P \geq C_{S_{H}}(P) \geq C_{S_{1}}(P)$, and thus $P \in \mathrm{Ob}\left(\mathcal{T}_{H}\right)$. So $\mathcal{F}_{H}$ is saturated by Proposition 3.6.

(d) We must prove that $\left|\mathcal{T}_{H}\right|$ is homotopy equivalent to a covering space of $|\mathcal{T}|$. Let $\mathcal{T}_{H}^{\prime} \subseteq \mathcal{T}$ be the subcategory with $\operatorname{Ob}\left(\mathcal{T}_{H}^{\prime}\right)=\operatorname{Ob}(\mathcal{T})$, and where $\operatorname{Mor}_{\mathcal{T}_{H}^{\prime}}(P, Q)$ is the set of all $\varphi \in \operatorname{Mor}_{\mathcal{T}}(P, Q)$ such that $\Phi(\varphi) \in H$. By Proposition A.4, $\left|\mathcal{T}_{H}^{\prime}\right|$ is homotopy equivalent to the covering space of $|\mathcal{T}|$ with fundamental group $\Phi^{-1}(H)$. Condition (1) in Proposition A.4 follows by point (a) here.

It remains to prove that $\left|\mathcal{T}_{H}^{\prime}\right| \simeq\left|\mathcal{T}_{H}\right|$. For all $P, Q \in \mathrm{Ob}\left(\mathcal{T}_{H}^{\prime}\right)$, all $\varphi \in \operatorname{Mor}_{\mathcal{T}_{H}^{\prime}}(P, Q)$, and all $g \in P, \varphi \circ \varepsilon_{P, P}(g) \circ \varphi^{-1}=\varepsilon_{Q, Q}(\rho(\varphi)(g))$ by axiom $(\mathrm{C})$, and thus $\Phi(\varphi) \in$ $H$ conjugates $\Phi\left(\varepsilon_{P, P}(g)\right)$ to $\Phi\left(\varepsilon_{Q, Q}(\rho(\varphi)(g))\right)$. In particular, $g \in S_{H}$ if and only if $\rho(\varphi)(g) \in S_{H}$, and thus $\rho(\varphi)\left(P \cap S_{H}\right) \leq Q \cap S_{H}$. So by Lemma 3.2(a), there is a unique restriction map

$$
r_{P, Q}^{H}: \operatorname{Mor}_{\mathcal{T}_{H}^{\prime}}(P, Q) \longrightarrow \operatorname{Mor}_{\mathcal{T}_{H}}\left(P \cap S_{H}, Q \cap S_{H}\right) .
$$

Define a retraction functor $r^{H}: \mathcal{T}_{H}^{\prime} \longrightarrow \mathcal{T}_{H}$ by sending an object $P$ to $P \cap S_{H}$, and sending $\psi \in \operatorname{Mor}_{\mathcal{T}}(P, Q)$ to $r_{P, Q}^{H}(\psi)$. The inclusion morphisms define a natural transformation of functors from incl $\circ r^{H}$ to $\operatorname{Id}_{\mathcal{T}_{H}^{\prime}}$, and thus $\left|\mathcal{T}_{H}^{\prime}\right| \simeq\left|\mathcal{T}_{H}\right|$.

We next look at the cohomology of the nerve of a transporter system $\mathcal{T}$. By Proposition A.3(b), for any abelian group $A$ with action of $\pi_{1}(|\mathcal{T}|), H^{*}(|\mathcal{T}| ; A) \cong \underbrace{\lim ^{*}}_{\mathcal{T}}(\Phi)$ for a certain functor $\Phi: \mathcal{T}^{\mathrm{op}} \longrightarrow A \mathrm{~b}$ which sends all objects to $A$. So we must study 
the higher limits of functors on $\mathcal{T}$. A more general discussion of higher limits is given in the appendix.

When $(S, \mathcal{F}, \mathcal{T})$ is a transporter system, the orbit category $\mathcal{O}(\mathcal{T})$ of $\mathcal{T}$ is defined to be the category whose objects are the objects of $\mathcal{T}$, and where

$$
\operatorname{Mor}_{\mathcal{O}(\mathcal{T})}(P, Q)=\varepsilon_{Q, Q}(Q) \backslash \operatorname{Mor}_{\mathcal{T}}(P, Q) .
$$

When $\mathcal{T}=\mathcal{T}_{S}(G)$ is the category of a finite group $G$ with Sylow subgroup $S$, then $\mathcal{O}(\mathcal{T})$ is the usual orbit category of $G$ :

$$
\operatorname{Mor}_{\mathcal{O}(\mathcal{T})}(P, Q)=Q \backslash N_{G}(P, Q) \cong \operatorname{map}_{G}(G / P, G / Q) .
$$

The relation between higher limits of $p$-local functors on $\mathcal{T}$ and on $\mathcal{O}(\mathcal{T})$ (i.e., functors which take values in $\mathbb{Z}_{(p)}$-modules) is described in the following lemma.

Lemma 4.2. Fix a transporter system $(S, \mathcal{F}, \mathcal{T})$, and let $\Phi: \mathcal{T}^{\mathrm{op}} \longrightarrow \mathbb{Z}_{(p)}$-mod be any functor. Then there is a spectral sequence

$$
E_{2}^{i j}={\underset{\mathcal{O}(\mathcal{T})}{\lim }}^{i}\left(H^{j}(-; \Phi(-))\right) \Longrightarrow{\underset{\mathcal{T}}{\lim ^{i+j}}}^{i+\Phi)}
$$

Proof. This is a special case of Proposition A.11.

The reason for working with higher limits over $\mathcal{O}(\mathcal{T})$ instead of looking directly at higher limits over $\mathcal{T}$ is that certain techniques developed when studying higher limits over other orbit categories also apply to this situation. We refer to the appendix for the definition of graded groups $\Lambda^{*}(G ; M)$, when $G$ is any finite group and $M$ is a $\mathbb{Z}_{(p)}$-module (and the prime $p$ is understood). Their relation to limits over $\mathcal{O}(\mathcal{T})$ is described by the following lemma.

Lemma 4.3. Fix a transporter system $(S, \mathcal{F}, \mathcal{T})$, and let

$$
\Phi: \mathcal{O}(\mathcal{T})^{\mathrm{op}} \longrightarrow \mathbb{Z}_{(p)} \bmod
$$

be any functor which vanishes except on the $\mathcal{F}$-conjugacy class of one subgroup $Q \in$ $\mathrm{Ob}(\mathcal{T})$. Then

$$
{\underset{\mathcal{O}(\mathcal{T})}{*}}^{*}(\Phi) \cong \Lambda^{*}\left(\operatorname{Aut}_{\mathcal{O}(\mathcal{T})}(Q) ; \Phi(Q)\right)
$$

Proof. Since the result is independent of the choice of $Q$ in its $\mathcal{F}$-conjugacy class, we can assume that $Q$ is fully normalized. Set $\Gamma=\operatorname{Aut}_{\mathcal{O}(\mathcal{T})}(Q)$, and set $\Sigma=\varepsilon_{Q, Q}\left(N_{S}(Q)\right) / Q \in$ $\operatorname{Syl}_{p}(\Gamma)$.

Let $\mathcal{O}_{p}(\Gamma)$ be the $p$-subgroup orbit category: the category whose objects are the $p$ subgroups of $\Gamma$, and with morphism sets $\operatorname{Mor}_{\mathcal{O}_{p}(\Gamma)}(P, Q)=Q \backslash N_{\Gamma}(P, Q)$. By definition, for any $\mathbb{Z}_{(p)}[\Gamma]$-module $M$,

$$
\Lambda^{*}(\Gamma ; M)={\underset{\mathcal{O}}{\mathcal{O}_{p}(\Gamma)}}^{\lim }\left(F_{M}\right) \quad \text { where } \quad F_{M}(P)= \begin{cases}M & \text { if } P=1 \\ 0 & \text { if } P \neq 1 .\end{cases}
$$

(See the discussion before Proposition A.2.) Let $\mathcal{O}_{\Sigma}(\Gamma) \subseteq \mathcal{O}_{p}(\Gamma)$ be the full subcategory with objects the subgroups of $\Sigma$; this is clearly a subcategory equivalent to $\mathcal{O}_{p}(\Gamma)$.

Define a functor

$$
\alpha: \mathcal{O}_{\Sigma}(\Gamma) \longrightarrow \mathcal{O}(\mathcal{T})
$$


as follows. For each subgroup $P / Q \leq \Sigma$, set $\alpha(P / Q)=P$. For each $P / Q, P^{\prime} / Q \leq \Sigma$ and each $\gamma \in \operatorname{Aut}_{\mathcal{T}}(Q)$ (i.e., $\left.Q \gamma \in \Gamma\right)$ such that $P^{\prime} \gamma \in \operatorname{Mor}_{\mathcal{O}(\Gamma)}\left(P / Q, P^{\prime} / Q\right)$, $\gamma$ extends to a unique morphism $\varphi \in \operatorname{Mor}_{\mathcal{T}}\left(P, P^{\prime}\right)$ by axiom (II), and we set

$$
\alpha(Q \gamma)=P^{\prime} \varphi \in \operatorname{Mor}_{\mathcal{O}(\mathcal{T})}\left(P, P^{\prime}\right) .
$$

We want to apply Proposition A.2 ([BLO3, Proposition 4.3]) to this functor $\alpha$. Point (a) $\left(\Gamma \cong \operatorname{End}_{\mathcal{O}(\mathcal{T})}(Q)\right)$ holds by construction. Point (c) follows from Lemmas 3.8 and A.8(b): all morphisms in $\mathcal{O}(\mathcal{T})$ are epimorphisms in the categorical sense since all morphisms in $\mathcal{T}$ are epimorphisms. It remains to check the other two hypotheses.

(b) For each $P \in \mathrm{Ob}(\mathcal{O}(\mathcal{T}))$ such that $P$ and $Q$ are not $\mathcal{T}$-isomorphic, all isotropy subgroups of the $\Gamma$-action on $\operatorname{Mor}_{\mathcal{O}(\mathcal{T})}(Q, P)$ (via right composition) are nontrivial p-subgroups. For $f \in \operatorname{Mor}_{\mathcal{T}}(Q, P)$, the isotropy subgroup of $[f] \in \operatorname{Mor}_{\mathcal{O}(\mathcal{T})}(Q, P)$ is the subgroup of all classes $[\alpha]$ for $\alpha \in \operatorname{Aut}_{\mathcal{T}}(Q)$ such that $f \circ \alpha=\varepsilon_{P, P}(x) \circ f$ for some $x \in P$. By Lemma 3.2, for any given $x \in P$, there is at most one $\alpha$ which satisfies this equation, and there is such $\alpha$ if and only if

$$
\rho(f)(Q)=\rho\left(\varepsilon_{P, P}(x) \circ f\right)(Q)=x \cdot \rho(f)(Q) \cdot x^{-1} .
$$

Thus the stabilizer subgroup of $[f]$ is isomorphic to $N_{P}(\rho(f)(Q)) / Q$, which is a nontrivial $p$-group since $\rho(f)(Q) \supsetneqq P$.

(d) For any $P / Q \leq \Sigma$, any $R \in \mathrm{Ob}(\mathcal{O}(\mathcal{T}))$, and any $\varphi \in \operatorname{Mor}_{\mathcal{O}(\mathcal{T})}(Q, R)$ which is $P / Q$-invariant, there is some $\bar{\varphi} \in \operatorname{Mor}_{\mathcal{O}(\mathcal{T})}(P, R)$ such that $\varphi=\bar{\varphi} \circ \iota_{Q, P}$. This follows from axiom (II).

Thus, by Proposition A.2, the natural map

$$
\underset{\mathcal{O}(\mathcal{T})}{\lim ^{*}}(\Phi) \stackrel{\alpha^{*}}{\cong}{\underset{\mathcal{O}}{\mathcal{O}_{\Sigma}(\Gamma)}}^{*}(\Phi \circ \alpha)=\Lambda^{*}(\Gamma ; \Phi(Q))
$$

is an isomorphism.

Lemma 4.3 can now be combined with results in [JMO] about the functors $\Lambda^{*}(-;-)$, to obtain the following corollary.

Corollary 4.4. Fix a transporter system $(S, \mathcal{F}, \mathcal{T})$.

(a) Assume

$$
\Phi: \mathcal{O}(\mathcal{T})^{\mathrm{op}} \longrightarrow \mathbb{Z}_{(p)}-\bmod
$$

has the property that for all $P \in \mathrm{Ob}(\mathcal{T})$ such that $\Phi(P) \neq 0$, there is an element of order $p$ in $\operatorname{Aut}_{\mathcal{O}(\mathcal{T})}(P)$ which acts trivially on $\Phi(P)$. Then

$$
{\underset{\mathcal{O}(\mathcal{T})}{\lim }}^{*}(\Phi)=0
$$

(b) Assume

$$
\Psi: \mathcal{T}^{\mathrm{op}} \longrightarrow \mathbb{Z}_{(p)} \text {-mod }
$$

has the property that for every fully centralized subgroup $P \in \mathrm{Ob}(\mathcal{T})$ such that $\Psi(P) \neq 0$, there is $g \in C_{S}(P) \backslash P$ such that $\varepsilon_{P, P}(g)$ acts trivially on $\Psi(P)$. Then ${\underset{\lim }{\mathcal{T}}}^{*}(\Psi)=0$.

Proof. By [JMO, Proposition 5.5], for any finite group $\Gamma$ and any $\mathbb{Z}_{(p)}[\Gamma]$-module $M$ such that some element $g \in \Gamma$ of order $p$ acts via the identity on $M, \Lambda^{*}(\Gamma ; M)=0$. In particular, by the hypothesis in $(\mathrm{a}), \Lambda^{*}\left(\operatorname{Aut}_{\mathcal{O}(\mathcal{T})}(P) ; \Phi(P)\right)=0$ for each $P \in \operatorname{Ob}(\mathcal{T})$. 
Point (a) now follows from Lemma 4.3, together with an appropriate filtration of $\Phi$ and the long exact sequences in higher limits for extensions of functors on $\mathcal{O}(\mathcal{T})$.

Point (b) follows from (a), together with Lemma 4.2.

We can now look at consequences for the homology of $|\mathcal{T}|$.

Proposition 4.5. Fix a finite group $G$, a Sylow subgroup $S \in \operatorname{Syl}_{p}(G)$, and a full subcategory $\mathcal{T} \subseteq \mathcal{T}_{S}(G)$ whose set of objects is closed under $G$-conjugacy and overgroups. Let $A$ be an $\mathbb{Z}_{(p)}[G]$-module, regarded also as a functor from $\mathcal{T}$ to $\mathbb{Z}_{(p)}$-mod which sends all objects to $A$ and which sends a morphism $g \in N_{G}(P, Q)$ to the action of $g$ on $A$. Let $S_{1} \leq S$ be the subgroup of elements which act on $A$ via the identity. Assume, for all $P \leq S$ such that $C_{S_{1}}(P) \leq P$, that $P \in \mathrm{Ob}(\mathcal{T})$ (the action of $\mathcal{T}$ on $A$ is admissible in the sense of Definition 5.10). Then

$$
H^{*}(|\mathcal{T}| ; A) \cong \underbrace{\lim ^{*}}_{\mathcal{T}}(A) \cong H^{*}(G ; A) ;
$$

where the first group means cohomology with coefficients twisted via the natural homomorphism $\pi_{1}(|\mathcal{T}|) \longrightarrow G$.

Proof. The first isomorphism follows from Proposition A.3(b). To prove the second, let $\Phi_{0} \subseteq \Phi: \mathcal{T}_{S}(G) \longrightarrow \mathbb{Z}_{(p)}$-mod be the functors

$$
\Phi(P)=A \quad \text { for all } P \leq S \quad \text { and } \quad \Phi_{0}(P)= \begin{cases}A & \text { if } P \notin \mathrm{Ob}(\mathcal{T}) \\ 0 & \text { if } P \in \mathrm{Ob}(\mathcal{T}) .\end{cases}
$$

In both cases, $g \in N_{G}(P, Q)$ acts on $A$ via the $\mathbb{Z}_{(p)}[G]$-module structure.

Let $\mathcal{T}_{\{1\}}(G) \subseteq \mathcal{T}_{S}(G)$ be the full subcategory with the trivial subgroup as the only object. We claim that

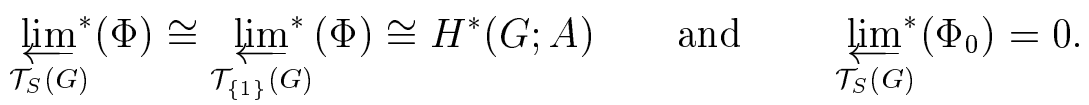

The first isomorphism holds because $\mathcal{T}_{S}(G)$ contains $\mathcal{T}_{\{1\}}(G)$ as a deformation retract (every morphism restricts to a unique automorphism of 1 ), and the second because functors from $\mathcal{T}_{\{1\}}(G)$ to abelian groups are the same as $\mathbb{Z}[G]$-modules. The last isomorphism follows from Lemma $4.4(\mathrm{~b})$, since whenever $P \notin \mathrm{Ob}(\mathcal{T}), C_{S_{1}}(P) \not \leq P$, and for any $g \in C_{S_{1}}(P) \backslash P, \varepsilon_{P, P}(g)$ acts trivially on $A$.

It now follows that

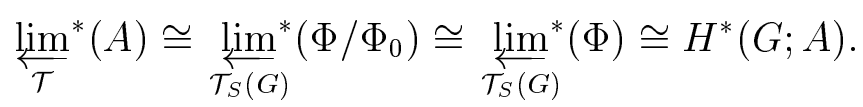

Here, the first isomorphism holds since $C^{*}(\mathcal{T} ; A) \cong C^{*}\left(\mathcal{T}_{S}(G) ; \Phi / \Phi_{0}\right)$ (the chain complexes defined in Lemma A.1).

As a second application of the results in this section, we show that the nerve of a transporter system $(S, \mathcal{F}, \mathcal{T})$ has the same $p$-completed homotopy type as the nerve of the associated linking system, in the sense of Proposition 3.7.

Proposition 4.6. Fix a transporter system $(S, \mathcal{F}, \mathcal{T})$ such that $\mathrm{Ob}(\mathcal{T})$ contains all subgroups $P \leq S$ which are $\mathcal{F}$-centric and $\mathcal{F}$-radical. Let $\mathcal{T}^{c}$ be the full subcategory with objects the $\mathcal{F}$-centric subgroups which are in $\mathrm{Ob}(\mathcal{T})$, and let $\mathcal{L}$ be the linking system associated to $\mathcal{T}^{c}$. Then the inclusion $\mathcal{T}^{c} \hookrightarrow \mathcal{T}$ and the projection $\mathcal{T}^{c} \longrightarrow \mathcal{L}$ induce homotopy equivalences

$$
|\mathcal{T}|_{p}^{\wedge} \simeq\left|\mathcal{T}^{c}\right|_{p}^{\wedge} \simeq|\mathcal{L}|_{p}^{\wedge}
$$


Proof. By [BLO1, Lemma 1.3], if $\tau: \widetilde{\mathcal{C}} \longrightarrow \mathcal{C}$ is any source regular functor (see Definition A.5) such that $\operatorname{Ker}\left(\tau_{\widetilde{\boldsymbol{c}}, \widetilde{c}}\right)$ is finite of order prime to $p$ for each $\widetilde{c} \in \mathrm{Ob}(\widetilde{\mathcal{C}})$, then $|\tau|$ induces an isomorphism from $H_{*}\left(|\widetilde{\mathcal{C}}| ; \mathbb{F}_{p}\right)$ to $H_{*}\left(|\mathcal{C}| ; \mathbb{F}_{p}\right)$, and hence a homotopy equivalence $|\widetilde{\mathcal{C}}| \simeq|\mathcal{C}|$. This is the situation for the projection of $\mathcal{T}^{c}$ onto $\mathcal{L}$ (by axiom (A2) for $\mathcal{T}$ ), and hence $\left|\mathcal{T}^{c}\right| \simeq|\mathcal{L}|$.

Let $\Phi: \mathcal{T}^{\mathrm{op}} \longrightarrow \mathrm{Ab}$ be the constant functor which sends all objects to $\mathbb{F}_{p}$ and all morphisms to the identity. Let $\Phi_{0} \subseteq \Phi$ be the subfunctor

$$
\Phi_{0}(P)= \begin{cases}\Phi(P) & \text { if } P \notin \mathrm{Ob}\left(\mathcal{T}^{c}\right) \\ 0 & \text { if } P \in \mathrm{Ob}\left(\mathcal{T}^{c}\right) .\end{cases}
$$

Then

$$
H^{*}\left(|\mathcal{T}| ; \mathbb{F}_{p}\right) \cong \underbrace{\lim _{\mathcal{T}}}_{\mathcal{T}}(\Phi) \quad \text { and } \quad H^{*}\left(|\mathcal{T}|,\left|\mathcal{T}^{c}\right| ; \mathbb{F}_{p}\right) \cong \underbrace{\lim _{\mathcal{T}}^{*}}_{\mathcal{T}}\left(\Phi_{0}\right) .
$$

For each $P \in \mathrm{Ob}(\mathcal{T}) \backslash \mathrm{Ob}\left(\mathcal{T}^{c}\right)$ which is fully centralized, and each $g \in C_{S}(P) \backslash P$, $\varepsilon_{P, P}(g)$ acts trivially on $\Phi(P)$. So $\lim ^{*}\left(\Phi_{0}\right)=0$ by Lemma 4.4(b). It follows that the inclusion of $\left|\mathcal{T}^{c}\right|$ into $|\mathcal{T}|$ is a mod $p$ homology equivalence, and hence induces a homotopy equivalence of $p$-completions.

Since a space is $p$-good if its $p$-completion is $p$-good [BK, Proposition I.5.2], and the $p$-completion of a linking system is $p$-good by [BLO2, Proposition 1.12], Proposition 4.6 implies that $|\mathcal{T}|$ is $p$-good whenever $\operatorname{Ob}(\mathcal{T})$ contains all $\mathcal{F}$-centric subgroups. In fact, the same argument as that used in the proof of [BLO2, Proposition 1.12] can be used in this situation (together with Proposition 3.10) to show directly that for any transporter system $\mathcal{T},|\mathcal{T}|$ is $p$-good.

We finish the section by describing one situation where an inclusion of transporter systems actually induces a homotopy equivalence between their uncompleted nerves.

Let $(S, \mathcal{F}, \mathcal{T})$ be a transporter system. Recall that a subgroup $P \in \operatorname{Ob}(\mathcal{T})$ is $\mathcal{T}$ radical if $O_{p}\left(\operatorname{Aut}_{\mathcal{T}}(P)\right)=\varepsilon_{P, P}(P)$. We let $\mathcal{T}^{r} \subseteq \mathcal{T}$ denote the full subcategory whose objects are the $\mathcal{T}$-radical subgroups of $S$.

The following proposition generalizes [BCGLO1, Theorem 3.5].

Proposition 4.7. Let $(S, \mathcal{F}, \mathcal{T})$ be a transporter system. Then $\left|\mathcal{T}^{r}\right| \simeq|\mathcal{T}|$. More generally, $\left|\mathcal{T}^{\prime}\right| \simeq|\mathcal{T}|$ for any full subcategory $\mathcal{T}^{\prime} \subseteq \mathcal{T}$ which contains $\mathcal{T}^{r}$.

Proposition 4.7 is an immediate consequence of the following lemma, whose proof is modelled on that of [BCGLO1, Proposition 3.11].

Lemma 4.8. Let $(S, \mathcal{F}, \mathcal{T})$ be a transporter system. Let $\mathcal{T}_{0} \subseteq \mathcal{T}$ be any full subcategory such that $\mathrm{Ob}\left(\mathcal{T}_{0}\right)$ is closed under $\mathcal{F}$-conjugacy. Let $P \leq S$ be maximal among those subgroups in $\mathrm{Ob}(\mathcal{T}) \backslash \mathrm{Ob}\left(\mathcal{T}_{0}\right)$, and let $\mathcal{T}_{1} \subseteq \mathcal{T}$ be the full subcategory whose objects are the objects in $\mathcal{T}_{0}$ together with all subgroups $\mathcal{F}$-conjugate to $P$. Assume furthermore that $P$ is not $\mathcal{T}$-radical. Then the inclusion of nerves $\left|\mathcal{T}_{0}\right| \subseteq\left|\mathcal{T}_{1}\right|$ is a homotopy equivalence.

Proof. Throughout this proof, "extensions" and "restrictions" of morphisms are taken as usual with respect to the inclusions $\iota_{P, Q}$ for $P \leq Q \leq S$. Also, for $\varphi \in \operatorname{Mor}_{\mathcal{T}}\left(Q, Q^{\prime}\right)$ and $R \leq Q$, we write $\varphi(R)=\rho(\varphi)(R) \leq Q^{\prime}$.

We must show that the inclusion functor $\mathcal{I}: \mathcal{T}_{0} \rightarrow \mathcal{T}_{1}$ induces a homotopy equivalence $\left|\mathcal{T}_{0}\right| \simeq\left|\mathcal{T}_{1}\right|$. By Quillen's Theorem A (see [Q]), it will be enough to prove that the undercategory $P^{\prime} \downarrow \mathcal{I}$ has contractible nerve (i.e., $\left|P^{\prime} \downarrow \mathcal{I}\right| \simeq *$ ) for each $P^{\prime}$ in $\mathcal{T}_{1}$. This is clear when $P^{\prime}$ is not isomorphic to $P$ (since $P^{\prime} \downarrow \mathcal{I}$ has initial object $\left(P^{\prime}, \mathrm{Id}\right)$ in that 
case), so it suffices to consider the case $P^{\prime}=P$. Since $P$ was arbitrarily chosen in its isomorphism class, we can also assume that $P$ is fully normalized.

By assumption, $P$ is not $\mathcal{T}$-radical, and thus $O_{p}\left(\operatorname{Aut}_{\mathcal{T}}(P)\right) \supsetneqq \varepsilon_{P, P}(P)$. Define

$$
\widehat{P}=\varepsilon_{P, P}^{-1}\left(O_{p}\left(\operatorname{Aut}_{\mathcal{T}}(P)\right)\right) .
$$

Since $P$ is fully normalized, $\varepsilon_{P, P}\left(N_{S}(P)\right) \in \operatorname{Syl}_{p}\left(\operatorname{Aut}_{\mathcal{T}}(P)\right)$ contains $O_{p}\left(\operatorname{Aut}_{\mathcal{T}}(P)\right)$. Hence $\varepsilon_{P, P}(\widehat{P})=O_{p}\left(\operatorname{Aut}_{\mathcal{T}}(P)\right)$, and so $\widehat{P} \supsetneqq P$.

Recall that the objects in $P \downarrow \mathcal{I}$ are the pairs $(Q, \varphi)$ for $Q \in \operatorname{Ob}\left(\mathcal{T}_{0}\right)$ and $\varphi \in$ $\operatorname{Mor}_{\mathcal{T}}(P, Q)$; and that $\operatorname{Mor}_{P \downarrow \mathcal{I}}((Q, \varphi),(R, \psi))$ is the set of all $\chi \in \operatorname{Mor}_{\mathcal{T}}(Q, R)$ such that $\chi \circ \varphi=\psi$. Consider the full subcategories

$$
\mathcal{C}_{2} \subseteq \mathcal{C}_{1} \subseteq \mathcal{C}_{0} \subseteq P \downarrow \mathcal{I}
$$

defined by setting

$$
\begin{aligned}
& \operatorname{Ob}\left(\mathcal{C}_{0}\right)=\{(Q, \varphi) \mid \varphi(P) \triangleleft Q\} \\
& \operatorname{Ob}\left(\mathcal{C}_{1}\right)=\{(Q, \varphi) \mid \varphi(P)=P \triangleleft Q\} \\
& \operatorname{Ob}\left(\mathcal{C}_{2}\right)=\{(Q, \varphi) \mid \varphi(P)=P \triangleleft Q, \widehat{P} \leq Q\} .
\end{aligned}
$$

We will prove that $|P \downarrow \mathcal{I}| \simeq\left|\mathcal{C}_{0}\right| \simeq\left|\mathcal{C}_{1}\right| \simeq\left|\mathcal{C}_{2}\right| \simeq *$.

Let $r_{1}: P \downarrow \mathcal{I} \longrightarrow \mathcal{C}_{0}$ be the retraction which sends $(Q, \varphi)$ to $\left(N_{Q}(\varphi(P)), \varphi^{\prime}\right)$, where $\varphi^{\prime} \in \operatorname{Mor}_{\mathcal{T}}\left(P, N_{Q}(\varphi(P))\right)$ is the restriction of $\varphi$, and which sends a morphism to its restriction. All of these restrictions are well defined by Lemma 3.2(a). There is a natural transformation of functors from incl $\circ r_{1}$ to the identity on $P \downarrow \mathcal{I}$ which sends an object $(Q, \varphi)$ to the inclusion $\iota_{N_{Q}(\varphi(P)), Q}$, and thus $|P \downarrow \mathcal{I}| \simeq\left|\mathcal{C}_{0}\right|$.

Now let $(Q, \varphi)$ be an object in $\mathcal{C}_{0}$, and set $P^{\prime}=\varphi(P) \triangleleft Q$ and $\varphi^{\prime}=\left.\varphi\right|_{P, P^{\prime}} \in$ $\operatorname{Iso}_{\mathcal{T}}\left(P, P^{\prime}\right)$. Since $P$ is fully normalized, $\varepsilon_{P, P}\left(N_{S}(P)\right) \in \operatorname{Syl}_{p}\left(\operatorname{Aut}_{\mathcal{T}}(P)\right)$, and hence the $p$-subgroup $\varphi^{\prime-1} \varepsilon_{P^{\prime}, P^{\prime}}(Q) \varphi^{\prime}$ is conjugate in $\operatorname{Aut}_{\mathcal{T}}(P)$ to a subgroup of $\varepsilon_{P, P}\left(N_{S}(P)\right)$. Let $\alpha \in \operatorname{Aut}_{\mathcal{T}}(P)$ be such that $\left(\alpha \varphi^{\prime-1}\right) \varepsilon_{P^{\prime}, P^{\prime}}(Q)\left(\alpha \varphi^{\prime-1}\right)^{-1}$ is contained in $\varepsilon_{P, P}\left(N_{S}(P)\right)$. By axiom (II) for $\mathcal{T}, \alpha \varphi^{\prime-1}$ extends to some morphism $\psi \in \operatorname{Mor}_{\mathcal{T}}\left(Q, N_{S}(P)\right)$ such that $\psi\left(P^{\prime}\right)=P$. Then $(\psi(Q), \psi \circ \varphi)$ is isomorphic to $(Q, \varphi)$; and $(\psi(Q), \psi \circ \varphi)$ is in $\mathcal{C}_{1}$ since $\psi \varphi(P)=P$. Thus every object in $\mathcal{C}_{0}$ is isomorphic to an object in $\mathcal{C}_{1}$, and this proves that $\left|\mathcal{C}_{0}\right| \simeq\left|\mathcal{C}_{1}\right|$.

Now define a retraction $r_{2}: \mathcal{C}_{1} \longrightarrow \mathcal{C}_{2}$ as follows. On objects, we set $r_{2}(Q, \varphi)=$ $\left(Q \widehat{P}, \iota_{Q, Q} \widehat{P}^{\circ} \varphi\right)$. Fix $\chi \in \operatorname{Mor}_{\mathcal{C}_{1}}((Q, \varphi),(R, \psi))$; then $\chi(P)=P$ since $\varphi(P)=P=\psi(P)$, so the restriction $\left.\chi_{0} \stackrel{\text { def }}{=} \chi\right|_{P, P} \in \operatorname{Aut}_{\mathcal{T}}(P)$ is defined. Since $\chi_{0}$ normalizes $\varepsilon_{P, P}(\widehat{P})=$ $O_{p}\left(\operatorname{Aut}_{\mathcal{T}}(P)\right)$, and since $\chi_{0}$ conjugates $\varepsilon_{P, P}(Q)$ to $\varepsilon_{P, P}(R)$ by Lemma 3.3, there is an extension $r_{2}(\chi) \in \operatorname{Mor}_{\mathcal{T}}(Q \widehat{P}, R \widehat{P})$ of $\chi_{0}$ by axiom (II), which is unique (and also an extension of $\chi$ ) by Lemma 3.8. Thus $r_{2}(\chi)$ is a morphism in $\mathcal{C}_{2}$ from $r_{2}(Q, \varphi)$ to $r_{2}(R, \psi)$, and the uniqueness of this extension implies that $r_{2}$ is a functor. There is a natural transformation of functors from incl $\circ r_{2}$ to $\operatorname{Id}_{\mathcal{C}_{1}}$ which sends an object $(Q, \varphi)$ to the morphism $\iota_{Q, Q} \widehat{P}$, and hence $\left|\mathcal{C}_{1}\right| \simeq\left|\mathcal{C}_{2}\right|$.

It remains to show that $\left|\mathcal{C}_{2}\right|$ is contractible. For any object $(Q, \varphi)$ in $\mathcal{C}_{2}$, axiom (II) can again be used to construct an extension of $\varphi$ to $\bar{\varphi} \in \operatorname{Mor}_{\mathcal{T}}(\widehat{P}, Q)$, thus a morphism in $\mathcal{C}_{2}$ from $\left(\widehat{P}, \iota_{P, \widehat{P}}\right)$ to $(Q, \varphi)$, and this morphism is unique by Lemma 3.8 . Hence $\left(\widehat{P}, \iota_{P, \widehat{P}}\right)$ is an initial object in $\mathcal{C}_{2}$, and $\left|\mathcal{C}_{2}\right| \simeq *$. 


\section{EXTENSIONS OF TRANSPORTER SYSTEMS}

We are now ready to study extensions of transporter systems and the resulting extensions of fusion systems. We first give a precise definition of what we mean by an extension of a transporter system. Afterwards, we show that any such extension is itself a transporter system, give conditions for when it is a linking system, and look at the obstruction theory for such extensions.

Definition 5.1. Let $(S, \mathcal{F}, \mathcal{T})$ be a transporter system. An extension $\mathcal{T}$ consists of a category $\tilde{\mathcal{T}}$ and a functor $\tau: \widetilde{\mathcal{T}} \longrightarrow \mathcal{T}$, such that for all $\bar{P}, \bar{Q} \in \mathrm{Ob}(\widetilde{\mathcal{T}})$,

(a) $\tau$ is a bijection on objects;

(b) $K_{\bar{P}} \stackrel{\text { def }}{=} \operatorname{Ker}\left[\tau_{\bar{P}, \bar{P}}: \operatorname{Aut}_{\widetilde{\mathcal{T}}}(\bar{P}) \longrightarrow \operatorname{Aut}_{\mathcal{T}}(\tau(\bar{P}))\right]$ is a finite p-group;

(c) $K_{\bar{P}}$ acts freely on $\operatorname{Mor}_{\tilde{\mathcal{T}}}(\bar{P}, \bar{Q})$ by right composition and $\tau$ is the orbit map of this action; and

(d) $K_{\bar{Q}}$ acts freely on $\operatorname{Mor}_{\widetilde{\mathcal{T}}}(\bar{P}, \bar{Q})$ by left composition, and $\tau$ is the orbit map of this action.

Conditions (a), (c), and (d) above are equivalent to saying that $\tau: \widetilde{\mathcal{T}} \longrightarrow \mathcal{T}$ is a source and target regular extension, in the sense of Definition A.5.

We want to show that every extension of a transporter system in this sense is again a transporter system; moreover, that an extension of $\mathcal{T}$ is equivalent to a transporter system $\widetilde{\mathcal{T}}$ with normal subgroup $A \triangleleft \widetilde{\mathcal{T}}$ such that $\widetilde{\mathcal{T}} / A \cong \mathcal{T}$. In order to show that $\widetilde{\mathcal{T}}$ is a transporter system, we need first to associate a finite $p$-group $\widetilde{S}$, and a fusion system $\widetilde{\mathcal{F}}$ over $\widetilde{S}$, to the category $\widetilde{\mathcal{T}}$.

Define $\widetilde{S}$ to be the pull-back of $\tau_{\bar{S}, \bar{S}}$ and $\varepsilon_{S, S}$ :

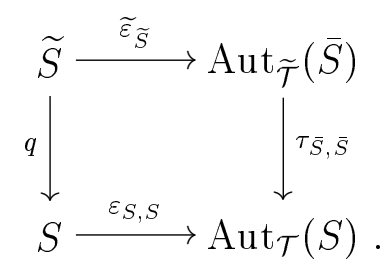

Thus $\widetilde{S}=\left\{(g, f) \in S \times \operatorname{Aut}_{\widetilde{\mathcal{T}}}(\bar{S}) \mid \varepsilon_{S, S}(g)=\tau(f)\right\}$. Let $q: \widetilde{S} \longrightarrow S$ and $\widetilde{\varepsilon}_{\widetilde{S}}: \widetilde{S} \longrightarrow$ Aut $_{\widetilde{\mathcal{T}}}(\bar{S})$ be the structure maps of the pull-back, so $q$ is surjective and $\widetilde{\varepsilon}_{\widetilde{S}}$ is injective. Set $A=\operatorname{Ker}(q)$, and set $\widetilde{P}=q^{-1}(P)$ for each $P \leq S$. So $A$ and $\widetilde{P}$ are subgroups of $\widetilde{S}$, and each $\widetilde{P}$ contains $A$. In fact, for each $P \in \mathrm{Ob}(\mathcal{T})$, the group $\widetilde{P}$ is an extension of the form

$$
1 \longrightarrow A \stackrel{i}{\longrightarrow} \widetilde{P} \stackrel{\left.q\right|_{\widetilde{P}}}{\longrightarrow} P \longrightarrow 1
$$

(including the case of $S$ ), where $i$ is the inclusion map. Since the functor $\tau$ is a bijection on objects and we defined a group $\widetilde{P} \leq \widetilde{S}$ for each $P \in \mathrm{Ob}(\mathcal{T})$, we will consider that the objects in $\widetilde{\mathcal{T}}$ are subgroups of $\widetilde{S}$ (all containing $A$ as a normal subgroup) and will denote by $\widetilde{P}$ the object in $\widetilde{\mathcal{T}}$ such that $\tau(\widetilde{P})=P$.

The pull-back square (1) also shows that $\widetilde{\varepsilon}_{\widetilde{S}}$ restricts to an isomorphism from $A$ to $\operatorname{Ker}\left(\tau_{\widetilde{S}}\right)$. In particular, $A$ is a $p$-group by condition (b) in Definition 5.1 , and so $\widetilde{S}$ is also a $p$-group. 
The following lifting lemma is the analogy for extensions of transporter systems to Lemmas 1.7 and Lemma 3.2.

Lemma 5.2. Let $\mathcal{T}$ be a transporter system and let $\widetilde{\mathcal{T}}$ be an extension of $\mathcal{T}$ by the p-group A. Fix morphisms $P \stackrel{\varphi}{\longrightarrow} Q \stackrel{\psi}{\longrightarrow} R$ in $\mathcal{T}$, and let $\widetilde{\psi \varphi} \in \operatorname{Mor}_{\widetilde{\mathcal{T}}}(\widetilde{P}, \widetilde{R})$ be any lifting of $\psi \circ \varphi$.

(a) For any lifting $\widetilde{\varphi} \in \operatorname{Mor}_{\widetilde{\mathcal{T}}}(\widetilde{P}, \widetilde{Q})$ of $\varphi$, there is a unique morphism $\widetilde{\psi} \in \operatorname{Mor}_{\widetilde{\mathcal{T}}}(\widetilde{Q}, \widetilde{R})$ such that $\tau(\widetilde{\psi})=\psi$ and $\widetilde{\psi} \circ \widetilde{\varphi}=\widetilde{\psi \varphi}$.

(b) For any lifting $\widetilde{\psi} \in \operatorname{Mor}_{\widetilde{\mathcal{T}}}(\widetilde{Q}, \widetilde{R})$ of $\psi$, there is a unique morphism $\widetilde{\varphi} \in \operatorname{Mor}_{\widetilde{\mathcal{T}}}(\widetilde{P}, \widetilde{Q})$ such that $\tau(\widetilde{\varphi})=\varphi$ and $\widetilde{\psi} \circ \widetilde{\varphi}=\widetilde{\psi \varphi}$.

Proof. These are special cases of Lemmas A.7(a) and A.8(a).

Next, we define the functors

$$
\mathcal{T}_{\mathrm{Ob}(\widetilde{\mathcal{T}})}(\widetilde{S}) \stackrel{\widetilde{\varepsilon}}{\longrightarrow} \widetilde{\mathcal{T}} \stackrel{\widetilde{\rho}}{\longrightarrow} \mathrm{Gps}
$$

which will give $\widetilde{\mathcal{T}}$ the structure of a transporter system. Notice that we already have a group monomorphism $\widetilde{\varepsilon}_{\widetilde{S}}: \widetilde{S} \longrightarrow \operatorname{Aut}_{\widetilde{\mathcal{T}}}(\widetilde{S})$, and we will be using it in the proofs of Lemmas 5.3 and 5.4.

For each $\widetilde{P}$, choose a lifting $\widetilde{\iota}_{\widetilde{P}} \in \operatorname{Mor}_{\widetilde{\mathcal{T}}}(\widetilde{P}, \widetilde{S})$ of $\iota_{P} \in \operatorname{Mor}_{\mathcal{T}}(P, S)$. These are chosen arbitrarily, except that we require $\widetilde{\iota}_{\widetilde{S}}=\operatorname{Id}_{\widetilde{S}}$ in $\widetilde{\mathcal{T}}$. (Recall that $\iota_{P}=\varepsilon_{P, P}(1)$.)

Lemma 5.3. For each $\widetilde{P}, \widetilde{Q} \in \mathrm{Ob}(\widetilde{\mathcal{T}})$ and each $\widetilde{x} \in N_{\widetilde{S}}(\widetilde{P}, \widetilde{Q})$, there is a unique morphism $\widetilde{\varepsilon}_{\widetilde{P}, \widetilde{Q}}(\widetilde{x})$ in $\widetilde{\mathcal{T}}$ that makes the following diagram commute:

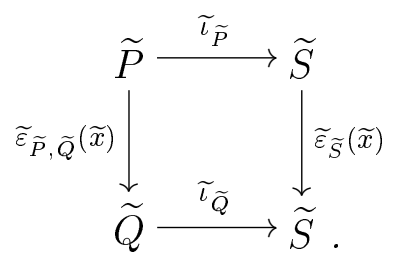

Furthermore, $\widetilde{\varepsilon}_{\widetilde{P}, \widetilde{P}}(A)=\operatorname{Ker}\left(\tau_{\widetilde{P}, \widetilde{P}}\right)$, and thus $\operatorname{Ker}\left(\tau_{\widetilde{P}, \widetilde{P}}\right) \cong A$.

Proof. The first statement follows immediately from lifting Lemma 5.2(b), applied with $\widetilde{\psi}=\widetilde{\iota}_{\widetilde{Q}}, \widetilde{\psi \varphi}=\widetilde{\varepsilon}_{\widetilde{S}}(\widetilde{x}) \circ \widetilde{\iota}_{\widetilde{P}}$, and $\varphi=\varepsilon_{P, P}(q(\widetilde{x}))$.

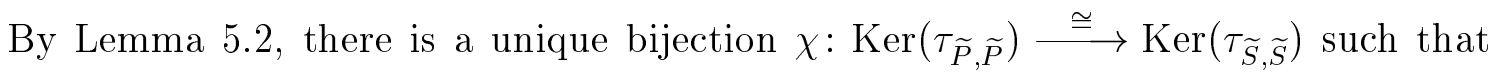
the square

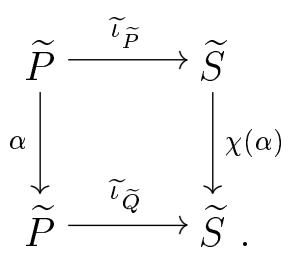

commutes for all $\alpha \in \operatorname{Ker}\left(\tau_{\widetilde{P}, \widetilde{P}}\right)$. Since $\chi\left(\widetilde{\varepsilon}_{\widetilde{P}, \widetilde{P}}(a)\right)=\widetilde{\varepsilon}_{\widetilde{S}}(a)$ for $a \in A$ by $(2)$, and $\widetilde{\varepsilon}_{\widetilde{S}}(A)=\operatorname{Ker}\left(\tau_{\widetilde{S}, \widetilde{S}}\right)$ by definition of $\widetilde{\varepsilon}_{\widetilde{S}}$, this shows that $\widetilde{\varepsilon}_{\widetilde{P}, \widetilde{P}}(A)=\operatorname{Ker}\left(\tau_{\widetilde{P}, \widetilde{P}}\right)$.

We thus get a well-defined map from $\operatorname{Mor}\left(\mathcal{T}_{\mathrm{Ob}(\widetilde{\mathcal{T}})}(\widetilde{S})\right)$ to $\operatorname{Mor}(\widetilde{\mathcal{T}})$ which sends $\widetilde{x}$ to $\widetilde{\varepsilon}_{\widetilde{P}, \widetilde{Q}}(\widetilde{x})$. By putting together two squares of the form $(2)$ we see that this defines a 
functor

$$
\widetilde{\varepsilon}: \mathcal{T}_{\mathrm{Ob}(\widetilde{\mathcal{T}})}(\widetilde{S}) \longrightarrow \widetilde{\mathcal{T}}
$$

which is the identity on objects. Notice that $\widetilde{\varepsilon} \widetilde{P}, \widetilde{Q}$ is defined so that the square

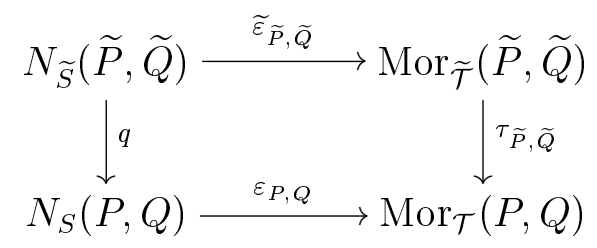

commutes in $\widetilde{\mathcal{T}}$, i.e., $\widetilde{\varepsilon_{\widetilde{P}}, \widetilde{Q}}$ is a lifting of $\varepsilon_{P, Q}$.

Lemma 5.4. For all $\widetilde{P}, \widetilde{Q} \in \mathrm{Ob}(\widetilde{\mathcal{T}})$, the map $\widetilde{\varepsilon_{\widetilde{P}}, \widetilde{Q}}: N_{\widetilde{S}}(\widetilde{P}, \widetilde{Q}) \longrightarrow \operatorname{Mor}_{\widetilde{\mathcal{T}}}(\widetilde{P}, \widetilde{Q})$ is injective.

Proof. Let $\widetilde{x}, \widetilde{y} \in N_{\widetilde{S}}(\widetilde{P}, \widetilde{Q})$ be such that $\widetilde{\varepsilon}_{\widetilde{P}, \widetilde{Q}}(\widetilde{x})=\widetilde{\varepsilon}_{\widetilde{P}, \widetilde{Q}}(\widetilde{y})$. Then

$$
\widetilde{\varepsilon}_{\widetilde{S}}(\widetilde{x}) \circ \widetilde{\iota}_{\widetilde{P}}=\widetilde{\iota}_{\widetilde{Q}} \circ \widetilde{\varepsilon}_{\widetilde{P}, \widetilde{Q}}(\widetilde{x})=\widetilde{\iota}_{\widetilde{Q}} \circ \widetilde{\varepsilon}_{\widetilde{P}, \widetilde{Q}}(\widetilde{y})=\widetilde{\varepsilon}_{\widetilde{S}}(\widetilde{y}) \circ \widetilde{\iota}_{\widetilde{P}}
$$

by (2). Also, by $(3), q(\widetilde{x})=q(\widetilde{y})$, so $\widetilde{\varepsilon}_{\widetilde{S}}(\widetilde{x})$ and $\widetilde{\varepsilon}_{\widetilde{S}}(\widetilde{y})$ are liftings of the same morphism $\varepsilon_{S, S}(q(\widetilde{x}))$. Thus $\widetilde{\varepsilon}_{\widetilde{S}}(\widetilde{x})=\widetilde{\varepsilon}_{\widetilde{S}}(\widetilde{y})$ by Lemma $5.2(\mathrm{a})$. But $\widetilde{\varepsilon}_{\widetilde{S}}$ is injective by construction (see (1)), so $\widetilde{x}=\widetilde{y}$.

Lemma 5.5. Given a morphism $f \in \operatorname{Mor}_{\widetilde{\mathcal{T}}}(\widetilde{P}, \widetilde{Q})$, there is a unique group homorphism $\varphi \in \operatorname{Hom}(\widetilde{P}, \widetilde{Q})$ such that the diagram

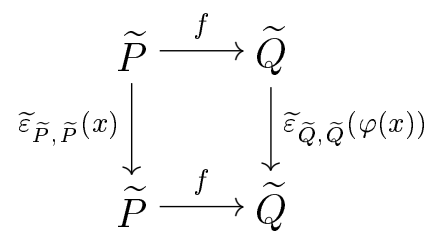

commutes for all $x \in \widetilde{P}$.

Proof. Set $\bar{x}=q(x)$. By axiom $(\mathrm{C})$ of the transporter system $\mathcal{T}$,

$$
\tau(f) \circ \varepsilon_{P, P}(\bar{x})=\varepsilon_{Q, Q}(\rho(\tau(f))(\bar{x})) \circ \tau(f),
$$

for all $\bar{x} \in P$. So the result follows from the lifting Lemma $5.2(\mathrm{a})$.

Now we can define the functor $\widetilde{\rho}: \widetilde{\mathcal{T}} \longrightarrow$ Gps to be the identity on objects, and to send a morphism $f \in \operatorname{Mor}_{\widetilde{\mathcal{T}}}(\widetilde{P}, \widetilde{Q})$ to the unique group homomorphism defined in Lemma 5.5. Again, by putting together commutative squares of the form (4), one sees that $\widetilde{\rho}$ is indeed a functor.

Let $\widetilde{\mathcal{F}}$ be the fusion system generated by the image of the functor $\widetilde{\rho}$. Thus when $\widetilde{P}, \widetilde{Q} \in \operatorname{Ob}(\widetilde{\mathcal{T}}), \operatorname{Mor}_{\widetilde{\mathcal{F}}}(\widetilde{P}, \widetilde{Q})$ is the image under $\widetilde{\rho}$ of $\operatorname{Mor}_{\tilde{\mathcal{T}}}(\widetilde{P}, \widetilde{Q})$. For arbitrary subgroups $R, R^{\prime} \leq \widetilde{S}, \operatorname{Mor}_{\widetilde{\mathcal{T}}}\left(R, R^{\prime}\right) \subseteq \operatorname{Inj}\left(R, R^{\prime}\right)$ is the set of all monomorphisms which are composites of restrictions of $\widetilde{\mathcal{F}}$-morphisms between objects in $\widetilde{\mathcal{T}}$.

Proposition 5.6. The category $\widetilde{\mathcal{T}}$, together with the maps $\widetilde{\varepsilon}_{\widetilde{P}, \widetilde{Q}}$ and the functor $\widetilde{\rho}$ defined in Lemmas 5.3 and 5.5, define a transporter system associated to $\widetilde{\mathcal{F}}$. In particular, $\widetilde{\mathcal{F}}$ is an $\operatorname{Ob}(\widetilde{\mathcal{T}})$-saturated fusion system. Furthermore, $A \triangleleft \widetilde{\mathcal{T}}$, and $(\widetilde{S} / A, \widetilde{\mathcal{F}} / A, \widetilde{\mathcal{T}} / S) \cong$ $(S, \mathcal{F}, \mathcal{T})$. 
Proof. The last statement is clear, and the saturation of $\widetilde{\mathcal{F}}$ follows from Proposition 3.6, once we know that $\widetilde{\mathcal{T}}$ is a transporter system. So we need only check that $\widetilde{\mathcal{T}}$ satisfies the axioms of Definition 3.1. Axiom (A1) follows from the conditions in Definition 5.1 for the extension $\widetilde{\mathcal{T}}$, and axiom (C) holds by construction of $\widetilde{\rho}$.

For each $\widetilde{P}, \widetilde{Q} \leq \widetilde{S}$, the injectivity of $\widetilde{\varepsilon_{\widetilde{P}}, \widetilde{Q}}$ was shown in Lemma 5.4. The composite $\widetilde{\rho}_{\widetilde{P}, \widetilde{Q}} \circ \widetilde{\varepsilon}_{\widetilde{P}, \widetilde{Q}}$ sends $g \in N_{\widetilde{S}}(\widetilde{P}, \widetilde{Q})$ to $c_{g} \in \operatorname{Hom}_{\widetilde{\mathcal{F}}}(\widetilde{P}, \widetilde{Q})$ by the construction of $\widetilde{\rho}$ using Lemma 5.5 (applied with $f=\varepsilon_{\widetilde{P}, \widetilde{Q}}(g)$ ). This proves axiom (B).

We next check axiom (A2). For each $\widetilde{P} \in \mathrm{Ob}(\widetilde{\mathcal{T}})$, set

$$
\widetilde{E}(\widetilde{P})=\operatorname{Ker}\left[\operatorname{Aut}_{\widetilde{\mathcal{T}}}(\widetilde{P}) \longrightarrow \operatorname{Aut}_{\widetilde{\mathcal{F}}}(\widetilde{P})\right] \text {. }
$$

Then $\tau_{\widetilde{P}, \widetilde{P}}(\widetilde{E}(\widetilde{P})) \leq E(P)$. So the free actions of $E(P)$ and $E(Q)$ on $\operatorname{Mor}_{\mathcal{T}}(P, Q)$ lift to free actions of $\widetilde{E}(\widetilde{P})$ and $\widetilde{E}(\widetilde{Q})$ on $\operatorname{Mor}_{\widetilde{\mathcal{T}}}(\widetilde{P}, \widetilde{Q})$. If $f, f^{\prime}$ are two morphisms such that $\widetilde{\rho}(f)=\widetilde{\rho}\left(f^{\prime}\right)$, then $\rho(\tau(f))=\rho\left(\tau\left(f^{\prime}\right)\right)$, so $\tau\left(f^{\prime}\right)=\tau(f) \circ \alpha$ for some $\alpha \in E(P)$ by axiom (A2) for $\mathcal{T}$. By the lifting Lemma 5.2(b), $\alpha$ lifts to a unique $\widetilde{\alpha} \in \operatorname{Aut}_{\widetilde{\mathcal{T}}}(\widetilde{P})$ such that $f^{\prime}=f \circ \widetilde{\alpha}$, and $\widetilde{\alpha} \in \widetilde{E}(\widetilde{P})$ since $\widetilde{\rho}\left(f^{\prime}\right)=\widetilde{\rho}(f)$.

Axiom (I) for $\widetilde{\mathcal{T}}\left(\widetilde{\varepsilon}_{\widetilde{S}, \widetilde{S}}(\widetilde{S}) \in \operatorname{Syl}_{p}\left(\operatorname{Aut}_{\widetilde{\mathcal{T}}}(\widetilde{S})\right)\right.$ follows from axiom (I) for $\mathcal{T}$.

Axiom (II) is a consequence of the same axiom applied to $\mathcal{T}$. Given a morphism $\varphi \in \operatorname{Mor}_{\widetilde{\mathcal{T}}}(\widetilde{P}, \widetilde{Q})$, and subgroups $\widetilde{P} \leq \widetilde{P}^{\prime} \leq N_{\widetilde{S}}(\widetilde{P})$ and $\widetilde{Q} \leq \widetilde{Q}^{\prime} \leq N_{\widetilde{S}}(\widetilde{Q})$ such that $\varphi \circ \widetilde{\varepsilon}_{\widetilde{P}, \widetilde{P}}\left(\widetilde{P}^{\prime}\right) \circ \varphi^{-1} \leq \widetilde{\varepsilon}_{\widetilde{Q}, \widetilde{Q}}\left(\widetilde{Q}^{\prime}\right)$, apply the functor $\tau: \widetilde{\mathcal{T}} \longrightarrow \mathcal{T}$ to the last inclusion. Then axiom (II) for $\mathcal{T}$ implies that there is some $f \in \operatorname{Mor}_{\tilde{\mathcal{T}}}\left(\widetilde{P}^{\prime}, \widetilde{Q}^{\prime}\right)$ such that $\left.\tau(f)\right|_{P, Q}=$ $\tau(\varphi)$. Hence by Lemma $5.3\left(\operatorname{Ker}\left(\tau_{\widetilde{P}, \widetilde{P}}\right)=\widetilde{\varepsilon}_{\widetilde{P}, \widetilde{P}}(A)\right)$, there is $a \in A$ such that $\left.f\right|_{\widetilde{P}, \widetilde{Q}}=$ $\varphi \circ \widetilde{\varepsilon}_{\widetilde{P}, \widetilde{P}}(a)$. So $\bar{\varphi} \stackrel{\text { def }}{=} f \circ \widetilde{\varepsilon}_{\widetilde{P}^{\prime}, \widetilde{P}^{\prime}}\left(a^{-1}\right)$ is an extension of $\varphi$.

We now look at the action of $\mathcal{T}$ on $A$ induced by an extension of transporter systems.

Lemma 5.7. Fix a transporter system $(S, \mathcal{F}, \mathcal{T})$, and an extension

$$
1 \longrightarrow A \longrightarrow \tilde{\mathcal{T}} \stackrel{\tau}{\longrightarrow} \mathcal{T} \longrightarrow 1
$$

Then this defines an action $\Phi: \operatorname{Mor}(\mathcal{T}) \longrightarrow \operatorname{Out}(A)$ of $\operatorname{Mor}(\mathcal{T})$ on $A$ as follows. For each $f \in \operatorname{Mor}_{\mathcal{T}}(P, Q)$, choose any $\widetilde{f} \in \operatorname{Mor}_{\widetilde{\mathcal{T}}}(\widetilde{P}, \widetilde{Q})$ such that $\tau(\widetilde{f})=f$, and set

$$
\widetilde{\Phi}(\widetilde{f})=\left.\widetilde{\rho}(\tilde{f})\right|_{A} \in \operatorname{Aut}(A) .
$$

Then the class $\Phi(f)=[\widetilde{\Phi}(\widetilde{f})] \in \operatorname{Out}(A)$ is well defined, independently of the choice of lifting $\tilde{f}$, and satisfies the following conditions.

(a) $\Phi(f) \circ \Phi\left(f^{\prime}\right)=\Phi\left(f \circ f^{\prime}\right)$ for any composable pair of morphisms $f, f^{\prime}$ in $\mathcal{T}$.

(b) For any lifting $\tilde{f} \in \operatorname{Mor}(\tilde{\mathcal{T}})$ of $f$, the following square commutes for all $a \in A$ :

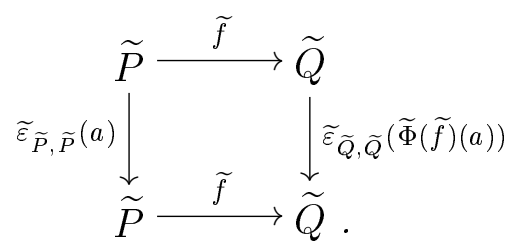

Proof. If $\widetilde{f}^{\prime}$ is another lifting of $f$, then there is some $a \in A$ such that $\widetilde{f}^{\prime}=\widetilde{f} \circ \widetilde{\varepsilon} \widetilde{P}, \widetilde{P}(a)$. Then $\widetilde{\rho}\left(\widetilde{f^{\prime}}\right)=\widetilde{\rho}(\widetilde{f}) \circ c_{a}$, and so $\left[\left.\widetilde{\rho}\left(\widetilde{f^{\prime}}\right)\right|_{A}\right]=\left[\left.\widetilde{\rho}(\widetilde{f})\right|_{A}\right]$ in $\operatorname{Out}(A)$. This proves that $\Phi(f)$ is well defined in $\operatorname{Out}(A)$, independently of the choice of lifting. 
Point (a) is immediate from the definition, and (b) follows from axiom (C) for $\tilde{\mathcal{T}}$.

Extensions of transporter systems in the sense of Definition 5.1 give one class of examples of extensions of categories which are both source regular and target regular, in the sense of Definition A.5. By that definition, an extension of the transporter system $\mathcal{T}$ is exactly the same as a source regular extension of $\mathcal{T}$ by a functor $F: \mathcal{T}^{\mathrm{op}} \longrightarrow \mathrm{Gps}_{*}$ such that $F$ sends all objects in $\mathcal{T}$ to finite $p$-groups and sends all morphisms to isomorphisms.

The general obstruction theory for constructing extensions of categories which are source and target regular and have abelian kernels is described in Proposition A.9 (with some remarks on the nonabelian case). The following proposition deals with the special case of extensions of transporter systems. Since any extension of a transporter system by a finite $p$-group can be factored as the composite of a sequence of extensions by abelian $p$-groups, the assumption that the kernel be abelian is not a major restriction.

Proposition 5.8. Fix a transporter system $(S, \mathcal{F}, \mathcal{T})$. Then extensions of $\mathcal{T}$ by a given finite abelian p-group $A$ are in one-to-one correspondence with actions $\Phi$ of $\pi_{1}(|\mathcal{T}|)$ on $A$, together with elements of $H^{2}(|\mathcal{T}| ; A)$ (with coefficients twisted by $\left.\Phi\right)$.

Proof. By Proposition A.3(a), an action of $\pi_{1}(|\mathcal{T}|)$ on $A$ correspond to maps from $\operatorname{Mor}(\mathcal{T})$ to $\operatorname{Aut}(A)$ which send composites to products and inclusions to the identity. Hence the action of $\pi_{1}(|\mathcal{T}|)$ on $A$ is described in Lemma A.7(c); and is the same as that defined by Lemma 5.7 by point (b) of that lemma. By Proposition A.9, the set of extensions which realize a given action of $\pi_{1}(|\mathcal{T}|)$ on $A$ are in one-to-one correspondence with $\lim ^{2}(K)$, where $K(P) \cong A$ for all $P$, and this group is isomorphic to $H^{2}(|\mathcal{T}| ; A)$ by Lemma A.3(b).

We now examine conditions for an extension of $\mathcal{T}$ to be a linking system, and also for its associated fusion system to be exotic. We first give an example of the complications which can arise if one doesn't impose extra conditions. Set $p=2$, and let $\mathcal{L}$ be the centric linking system of $A_{6}$. Since $\pi_{1}(|\mathcal{L}|) \cong \Sigma_{4} \underset{D_{8}}{*} \Sigma_{4}$, it surjects onto $A_{6} \cong P S L_{2}(9)$ and onto $P S L_{2}(7)$; in fact, onto $P S L_{2}(q)$ for any $q \equiv \pm 7(\bmod 16)$ which is a prime or the square of a prime. Let $|\mathcal{L}|$ act on $A=C_{2}^{7}$ via the faithful action of $A_{6}$ on $C_{2}^{4}$ and that of $P S L_{2}(7) \cong G L_{3}(2)$ on $C_{2}^{3}$. The resulting extension of $\mathcal{L}$ by $A$ is an exotic transporter system; but not very interesting, since it has too few objects to generate a saturated fusion system.

We now want to define the concept of an "admissible" action or extension of a transporter system, in order to avoid such examples. We first need one more lemma to motivate the precise conditions in the definition.

When $\tau: \widetilde{\mathcal{T}} \longrightarrow \mathcal{T}$ is an extension of $\mathcal{T}$ by $A$, we let

$$
\Phi_{P, Q}: \operatorname{Mor}_{\mathcal{T}}(P, Q) \longrightarrow \operatorname{Out}(A) \quad \text { and } \quad \Phi_{P}: E(P) \longrightarrow \operatorname{Out}(A)
$$

be the restrictions of the action $\Phi$ to the corresponding sets of morphisms. Recall that $E(P)=\operatorname{Ker}\left(\rho_{P, P}\right) \leq \operatorname{Aut}_{\mathcal{T}}(P)$, so $\Phi_{P}$ is a group homomorphism. We also write $\widetilde{E}(\widetilde{P})=\operatorname{Ker}\left(\widetilde{\rho}_{\widetilde{P}}, \widetilde{P}\right)$.

Lemma 5.9. Fix a transporter system $(S, \mathcal{F}, \mathcal{T})$, and an extension

$$
1 \longrightarrow A \longrightarrow \tilde{\mathcal{T}} \stackrel{\tau}{\longrightarrow} \mathcal{T} \longrightarrow 1
$$


Let $\widetilde{\mathcal{F}} \stackrel{\tau_{\mathcal{F}}}{\longrightarrow} \mathcal{F}$ and $\widetilde{S} \stackrel{q}{\longrightarrow} S$ be the extensions of fusion systems and p-groups associated to $\widetilde{\mathcal{T}}$. Set

$$
S_{1}=q\left(C_{\widetilde{S}}(A)\right)=\operatorname{Ker}\left[S \stackrel{\varepsilon_{S, S}}{\longrightarrow} \operatorname{Aut}_{\mathcal{T}}(S) \stackrel{\Phi_{S, S}}{\longrightarrow} \operatorname{Out}(A)\right] .
$$

Then the following hold for any subgroup $P \in \mathrm{Ob}(\mathcal{T})$, where $\widetilde{P}=q^{-1}(P)$.

(a) If $P$ is fully normalized in $\mathcal{F}$, then $\widetilde{E}(\widetilde{P}) \leq \widetilde{\varepsilon}_{\widetilde{P}, \widetilde{P}}\left(C_{\widetilde{S}}(\widetilde{P})\right)$ if and only if $\operatorname{Ker}\left(\Phi_{P}\right)$ is a p-group.

(b) If $\widetilde{P} \leq \widetilde{S}$ is any subgroup which is $\widetilde{\mathcal{F}}$-centric and $\widetilde{\mathcal{F}}$-radical, or more generally

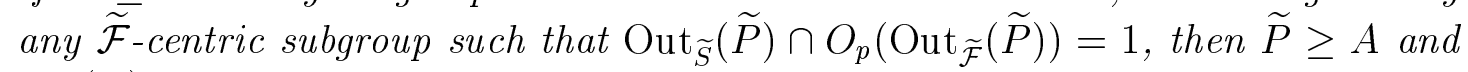
$C_{S_{1}}(P) \leq P$.

(c) If $C_{S_{1}}(P) \leq P$, then $\widetilde{P}$ is $\widetilde{\mathcal{F}}$-centric.

Proof. (a) Consider the following commutative diagram:

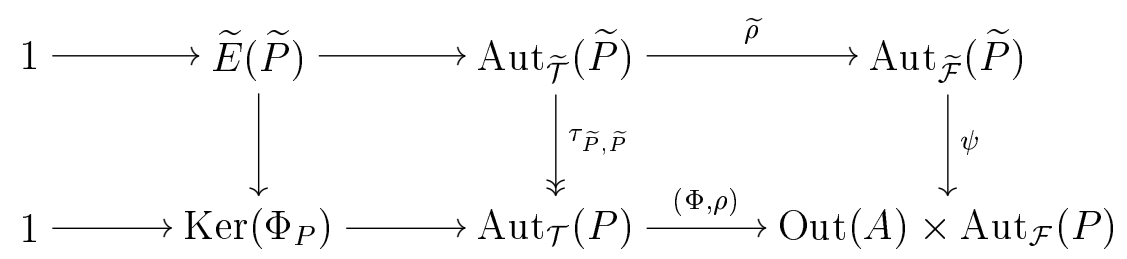

where $\psi(f)=\left(\left.f\right|_{A}, f / A\right)$ for $f \in \operatorname{Aut}_{\widetilde{\mathcal{F}}}(\widetilde{P})$. By Lemma 1.9(a), $\operatorname{Ker}(\psi)$ is a $p$-group. Hence for each $x \in \operatorname{Ker}\left(\Phi_{P}\right), x^{p^{k}} \in \tau_{\widetilde{P}} \widetilde{P}(\widetilde{E}(\widetilde{P}))$ for some $k$; and this implies that $\tau_{\widetilde{P}, \widetilde{P}}(\widetilde{E}(\widetilde{P}))$ has $p$-power index in $\operatorname{Ker}\left(\Phi_{P}\right)$. Since $\operatorname{Ker}\left(\tau_{\widetilde{P}, \widetilde{P}}\right) \cong A$ is a $p$-group, it now follows that $\widetilde{E}(\widetilde{P})$ is a $p$-group if and only if $\operatorname{Ker}\left(\Phi_{P}\right)$ is a $p$-group. In particular, if $\widetilde{E}(\widetilde{P}) \leq \varepsilon_{\widetilde{P}, \widetilde{P}}\left(C_{\widetilde{S}}(\widetilde{P})\right)$, then $\operatorname{Ker}\left(\Phi_{P}\right)$ is a $p$-group.

Now assume conversely that $\operatorname{Ker}\left(\Phi_{P}\right)$ is a $p$-group. We have just shown that $\widetilde{E}(\widetilde{P})$ is a $p$-group. Since $P$ is fully normalized, $\widetilde{P}$ is also fully normalized, and so axiom $\left(\mathrm{I}^{\prime}\right)$ for $\widetilde{\mathcal{T}}$ implies that $\widetilde{\varepsilon}_{\widetilde{P}, \widetilde{P}}\left(N_{\widetilde{S}}(\widetilde{P})\right)$ is a Sylow $p$-subgroup of $\operatorname{Aut}_{\widetilde{\mathcal{T}}}(\widetilde{P})$. Since a normal $p$-subgroup of a finite group is contained in all of its Sylow $p$-subgroups, this shows that

$$
\widetilde{E}(\widetilde{P}) \leq \widetilde{\varepsilon}_{\widetilde{P}, \widetilde{P}}\left(N_{\widetilde{S}}(\widetilde{P})\right)
$$

Furthermore, by axiom (C) for $\widetilde{\mathcal{T}}$, every element of $\widetilde{E}(\widetilde{P})$ commutes with every element of $\widetilde{\varepsilon}_{\widetilde{P}, \widetilde{P}}(\widetilde{P})$, and thus $\widetilde{E}(\widetilde{P}) \leq \widetilde{\varepsilon}_{\widetilde{P}, \widetilde{P}}\left(C_{\widetilde{S}}(\widetilde{P})\right)$.

(b) Assume $\widetilde{P} \leq \widetilde{S}$ is $\widetilde{\mathcal{F}}$-centric and $\operatorname{Out}_{\widetilde{S}}(\widetilde{P}) \cap O_{p}\left(\operatorname{Out}_{\widetilde{\mathcal{F}}}(\widetilde{P})\right)=1$, and consider the following diagram with exact rows:

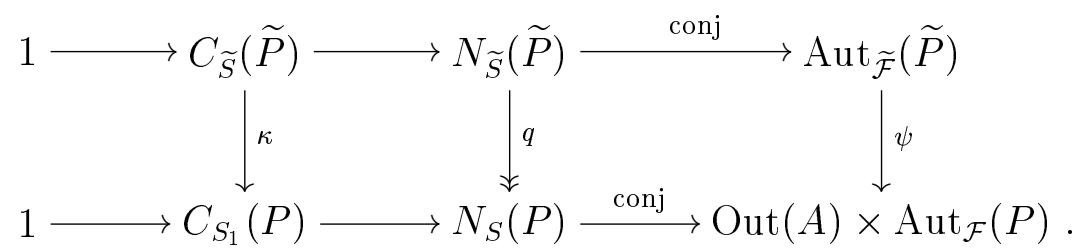

Then

$$
\left\{g \in N_{\widetilde{S}}(\widetilde{P}) \mid c_{g} \in \operatorname{Ker}(\psi)\right\} \leq C_{\widetilde{S}}(\widetilde{P}) \leq \widetilde{P}:
$$


the first inclusion since $\operatorname{Ker}(\psi) \leq O_{p}\left(\operatorname{Aut}_{\widetilde{\mathcal{F}}}(\widetilde{P})\right)$, and the second since $\widetilde{P}$ is $\widetilde{\mathcal{F}}$-centric. In particular, $A \leq \widetilde{P}$. Also, for all $g \in C_{S_{1}}(P)$, there is $\widetilde{g} \in N_{\widetilde{S}}(\widetilde{P})$ such that $q(\widetilde{g})=g$, $\widetilde{g} \in \widetilde{P}$ by $(6)$, and so $g \in P$.

(c) If $C_{S_{1}}(P) \leq P$, then $q\left(C_{\widetilde{S}}(\widetilde{P})\right) \leq P$, and so $\widetilde{P}$ is $\widetilde{\mathcal{F}}$-centric.

Conditions (b) and (c) above help to motivate the following definition.

Definition 5.10. Fix a transporter system $(S, \mathcal{F}, \mathcal{T})$.

(a) A homomorphism $\Phi: \pi_{1}(|\mathcal{T}|) \longrightarrow \Gamma$ is called admissible if, upon setting $S_{1}=$ $\operatorname{Ker}\left(\Phi \circ \varepsilon_{S, S}\right), P \leq S$ fully centralized in $\mathcal{F}$ and $C_{S_{1}}(P) \leq P$ imply $P \in \mathrm{Ob}(\mathcal{T})$.

(b) An action $\Phi: \pi_{1}(|\mathcal{T}|) \longrightarrow \operatorname{Out}(A)$ of $\mathcal{T}$ on a p-group $A$ is called admissible if the homomorphisms $\Phi$ is admissible.

(c) An extension $1 \rightarrow A \rightarrow \widetilde{\mathcal{T}} \rightarrow \mathcal{T} \rightarrow 1$ is called admissible if the action of $\mathcal{T}$ on $A$ defined in Lemma 5.7 is admissible.

Recall, for any fusion system $\mathcal{F}$ over $S$ and any $A \leq S$, the centralizer fusion system $C_{\mathcal{F}}(A)$ is the fusion system over $C_{S}(A)$ where for each $P, Q, \operatorname{Hom}_{C_{\mathcal{F}}(A)}(P, Q)$ is the set of those $f \in \operatorname{Hom}_{\mathcal{F}}(P, Q)$ which extend to $\bar{f} \in \operatorname{Hom}_{\mathcal{F}}(P A, Q A)$ such that $\left.\bar{f}\right|_{A}=\operatorname{Id}_{A}$ (see [BLO2, §2]). As a consequence of Lemma 5.9, when applied to admissible extensions, we now get:

Theorem 5.11. Fix a transporter system $(S, \mathcal{F}, \mathcal{T})$ and an admissible extension $1 \rightarrow$ $A \rightarrow \widetilde{\mathcal{T}} \rightarrow \mathcal{T} \rightarrow 1$, and let $\widetilde{S}$ and $\widetilde{\mathcal{F}}$ be the p-group and fusion system associated to $\widetilde{\mathcal{T}}$. Let $\Phi: \pi_{1}(|\mathcal{T}|) \longrightarrow \operatorname{Out}(A)$ be the action defined in Proposition 5.7, and let $\left(S_{1}, \mathcal{F}_{1}, \mathcal{T}_{1}\right)$ be the "kernel" transporter system of $\Phi$ in the sense of Proposition 4.1. Then the following hold.

(a) $\mathrm{Ob}(\widetilde{\mathcal{T}})$ contains all subgroups of $\widetilde{S}$ which are $\widetilde{\mathcal{F}}$-centric and $\widetilde{\mathcal{F}}$-radical. Moreover, every $\widetilde{\mathcal{F}}$-centric subgroup $\widetilde{P} \leq \widetilde{S}$ not in $\mathrm{Ob}(\widetilde{\mathcal{T}})$ is $\widetilde{\mathcal{F}}$-conjugate to some $\widetilde{P}^{\prime}$ such that $\operatorname{Out}_{\widetilde{S}}\left(\widetilde{P}^{\prime}\right) \cap O_{p}\left(\operatorname{Out}_{\widetilde{\mathcal{F}}}\left(\widetilde{P}^{\prime}\right) \neq 1\right.$.

(b) $\widetilde{\mathcal{F}}$ is a saturated fusion system.

(c) Let $\widetilde{\mathcal{L}}$ be the full subcategory of $\widetilde{\mathcal{T}}$ whose objects are those $\widetilde{\mathcal{F}}$-centric subgroups of $\widetilde{S}$ in $\operatorname{Ob}(\widetilde{\mathcal{T}})$. Then $\widetilde{\mathcal{L}}$ is a linking system if and only if $\operatorname{Ker}\left[\Phi_{P}: E(P) \longrightarrow \operatorname{Out}(A)\right]$ is a p-group for all $P \in \mathrm{Ob}(\mathcal{T})$ such that $\widetilde{P} \in \mathrm{Ob}(\widetilde{\mathcal{L}})$.

(d) If $\widetilde{\mathcal{F}}$ is the fusion system of a finite group $\widetilde{G}$, then $\mathcal{F}$ is the fusion system of $N_{\widetilde{G}}(A) / A$, and $\mathcal{F}_{1}$ is the fusion system of $C_{\widetilde{G}}(A) / A$. Thus if $\mathcal{F}_{1}$ is exotic (not the fusion system of any finite group), then so is $\widetilde{\mathcal{F}}$.

Proof. Point (a) follows immediately from Lemma 5.9(b), and point (b) then follows from Proposition 3.6.

By definition, $\widetilde{\mathcal{L}}$ is a linking system associated to $\widetilde{\mathcal{F}}$ if and only if $\widetilde{E}(\widetilde{P}) \leq \widetilde{\varepsilon}_{\widetilde{P}, \widetilde{P}}(Z(\widetilde{P}))$ for all $\widetilde{P} \in \operatorname{Ob}(\widetilde{\mathcal{L}})$, and $Z(\widetilde{P})=C_{\widetilde{S}}(\widetilde{P})$ for all such $\widetilde{P}$. Also, if this condition holds for any subgroup in the $\widetilde{\mathcal{F}}$-conjugacy class of $\widetilde{P}$, it also holds for $\widetilde{P}$. So (c) follows from Lemma 5.9(a).

It remains to prove $(\mathrm{d})$. By construction, $A \triangleleft \widetilde{\mathcal{F}}, \widetilde{\mathcal{F}} / A \cong \mathcal{F}$, and $C_{\widetilde{\mathcal{F}}}(A) / A \cong \mathcal{F}_{1}$. If $\widetilde{\mathcal{F}} \cong \mathcal{F}_{\widetilde{S}}(\widetilde{G})$ for some finite group $\widetilde{G}$, then since $A \triangleleft \widetilde{\mathcal{F}}, \widetilde{\mathcal{F}}$ is also the fusion system of 
$N_{\widetilde{G}}(A)$. Thus $\mathcal{F}$ is the fusion system of $N_{\widetilde{G}}(A) / A$. Also, by definition, a morphism in $\widetilde{\mathcal{F}}$ lies in $C_{\widetilde{\mathcal{F}}}(A)$ if and only if it is conjugation by an element in $C_{\widetilde{G}}(A)$. Hence $C_{\widetilde{\mathcal{F}}}(A)$ is the fusion system of $C_{\widetilde{G}}(A)$, and so $\mathcal{F}_{1}$ is the fusion system of $C_{\widetilde{G}}(A) / A$.

We finish the section with some tools which are useful when computing the obstruction groups which appear in Proposition 5.8.

Lemma 5.12. Fix a transporter system $(S, \mathcal{F}, \mathcal{T})$, a finite abelian p-group $A$, and an admissible action $\Phi: \mathcal{T} \longrightarrow \operatorname{Aut}(A)$. Set $S_{1}=\operatorname{Ker}\left(\Phi \circ \varepsilon_{S, S}\right) \triangleleft S$, and assume that $P \in \mathrm{Ob}(\mathcal{T})$ implies $P \cap S_{1} \in \mathrm{Ob}(\mathcal{T})$. Let $\mathcal{T}_{1} \subseteq \mathcal{T}$ be the transporter subsystem of Proposition 4.1: $\mathrm{Ob}\left(\mathcal{T}_{1}\right)$ is the set of subgroups of $S_{1}$ which are in $\mathrm{Ob}(\mathcal{T})$, and $\operatorname{Mor}\left(\mathcal{T}_{1}\right)$ is the set of morphisms $\psi$ in $\mathcal{T}$ between objects of $\mathcal{T}_{1}$ such that $\Phi(\psi)=1$. Then there is a spectral sequence

$$
E_{2}^{i j}=H^{i}\left(\Gamma ; H^{j}\left(\left|\mathcal{T}_{1}\right| ; A\right)\right) \Longrightarrow H^{i+j}(|\mathcal{T}| ; A),
$$

where $\Gamma=\operatorname{Im}(\Phi) \leq \operatorname{Aut}(A)$. Here, $H^{*}\left(\left|\mathcal{T}_{1}\right| ; A\right)$ is ordinary cohomology with untwisted coefficients. The action of $\Gamma$ on $H^{j}\left(\left|\mathcal{T}_{1}\right| ; A\right)$ is induced by the action of $\Gamma$ on $A$, together with the homotopy action of $\Gamma \cong \operatorname{Aut}_{\mathcal{T}}\left(S_{1}\right) / \operatorname{Aut}_{\mathcal{T}_{1}}\left(S_{1}\right)$ on $\left|\mathcal{T}_{1}\right|$ via restrictions.

Proof. This is the spectral sequence induced by the fibration sequence

$$
\tilde{X} \longrightarrow E \Gamma \times_{\Gamma} \widetilde{X} \longrightarrow B \Gamma,
$$

where $\tilde{X}$ denotes the covering space of $|\mathcal{T}|$ with fundamental group $\operatorname{Ker}(\Phi)$. By Proposition $4.1(\mathrm{~d}), \widetilde{X} \simeq\left|\mathcal{T}_{1}\right|$. The action of $\Gamma$ on $\widetilde{X}$ induces the homotopy action on $\left|\mathcal{T}_{1}\right|$ described above via the construction in the proof of Propositions 4.1(d) and A.4.

In the above situation, if one wants to determine the action of $\Gamma$ on $H^{*}\left(\left|\mathcal{T}_{1}\right| ; A\right)$ in concrete situations where the actions on $\left|\mathcal{T}_{1}\right|$ and on $A$ are both nontrivial, then more precision is needed when formulating the above statement. But this formulation suffices for our purposes here.

The following is an immediate corollary to Lemma 5.12.

Corollary 5.13. Let $(S, \mathcal{F}, \mathcal{T}), \Phi: \mathcal{T} \longrightarrow \operatorname{Aut}(A), S_{1} \triangleleft S$, and $\mathcal{T}_{1} \subseteq \mathcal{T}$ be as in Lemma 5.12, and set $\Gamma=\operatorname{Im}(\Phi) \leq \operatorname{Aut}(A)$. Assume, furthermore, that $H^{i}\left(\left|\mathcal{T}_{1}\right| ; \mathbb{F}_{p}\right)=$ 0 for $i=1,2$. Then $H^{i}(|\mathcal{T}| ; A) \cong H^{i}(\Gamma ; A)$ for $i=1,2$. Thus there is a bijective correspondence between extensions of $\mathcal{T}$ by $A$ and group extensions of $\Gamma$ by $A$ (with the given actions of $\mathcal{T}$ and $\Gamma$ on $A$ ).

\section{EXAMPLES}

We first look at conditions which imply that an admissible extension of the transporter category of a finite group (or one of its full subcategories) is again the transporter category of a finite group. Afterwards, we give some examples of "exotic" extension, where the target is the transporter category of a finite group but the source is not.

Recall that for any transporter system $(S, \mathcal{F}, \mathcal{T})$,

$$
\theta: \operatorname{Mor}(\mathcal{T}) \longrightarrow \pi_{1}(|\mathcal{T}|)
$$

is the map which sends $\varphi \in \operatorname{Mor}_{\mathcal{T}}(P, Q)$ to the loop $\iota_{Q, S} \cdot \varphi^{\cdot} \iota_{P, S}{ }^{-1}$ (composed from right to left). When $\mathcal{T}=\mathcal{T}_{S}(G)$ for some finite group $G$ and some $S \in \operatorname{Syl}_{p}(G)$, there is also a map $\operatorname{Mor}(\mathcal{T}) \longrightarrow G$ induced by the inclusions of the transporter sets $\operatorname{Mor}_{\mathcal{T}}(P, Q)=$ $N_{G}(P, Q)$ into $G$. This map clearly sends composites to products and inclusions $1 \in$ 
$N_{G}(P, S)$ to the identity. Hence by the universality property of $\theta$ (Proposition A.3(a)), there is a unique homomorphism

$$
\eta_{G}: \pi_{1}\left(\left|\mathcal{T}_{S}(G)\right|\right) \longrightarrow G
$$

such that $\eta_{G}(\theta(x))=x$ for all $P, Q \leq S$ and all $x \in N_{G}(P, Q)$. To keep the notation simple, we also write $\eta_{G}$ for the analogous homomorphism defined on $\pi_{1}(|\mathcal{T}|)$ when $\mathcal{T}$ is a full subcategory of $\mathcal{T}_{S}(G)$ which contains $S$.

Proposition 6.1. Fix a finite group $G$, a Sylow subgroup $S \in \operatorname{Syl}_{p}(G)$, and a set $\mathcal{H}$ of subgroups of $S$ which is closed under $G$-conjugacy and overgroups. Let

$$
1 \longrightarrow A \longrightarrow \tilde{\mathcal{T}} \stackrel{\tau}{\longrightarrow} \mathcal{T}_{\mathcal{H}}(G) \longrightarrow 1
$$

be an admissible extension where $A$ is a finite p-group. Assume furthermore that the homomorphism

$$
\Phi: \pi_{1}\left(\left|\mathcal{T}_{\mathcal{H}}(G)\right|\right) \longrightarrow \operatorname{Out}(A)
$$

of Lemma 5.7 factors through $G$ : that $\Phi=\Phi^{\prime} \circ \eta_{G}$ for some $\Phi^{\prime} \in \operatorname{Hom}(G, \operatorname{Out}(A))$. Then there is an extension of groups

$$
1 \longrightarrow A \longrightarrow \widetilde{G} \stackrel{\chi}{\longrightarrow} G \longrightarrow 1
$$

such that $\widetilde{\mathcal{T}}$ is a full subcategory of $\mathcal{T}_{\chi^{-1}(S)}(\widetilde{G})$.

Proof. Assume first that $A$ is abelian. In this case, by Proposition 5.8, the extensions of $\mathcal{T}=\mathcal{T}_{\mathcal{H}}(G)$ which realize the given action $\Phi$ are in bijective correspondence with elements of $H^{2}(|\mathcal{T}| ; A)$. By Proposition $4.5, \eta_{G}$ induces an isomorphism

$$
\eta_{G}^{*}: H^{*}(G ; A) \stackrel{\cong}{\longrightarrow} H^{*}(|\mathcal{T}| ; A) .
$$

Furthermore, by the construction of the cohomology invariants, $\eta_{G}^{*}$ sends the element in $H^{2}(G ; A)$ which describes a group extension to the element of $H^{2}(|\mathcal{T}| ; A) \cong{\underset{\mathcal{T}}{\lim ^{2}}}^{2}(A)$ which describes the extension of categories. Thus each extension of categories is induced by an extension of groups, which is what we wanted to prove.

Now assume $A$ is nonabelian, and set $\overline{\mathcal{T}}=\widetilde{\mathcal{T}} / Z(A)$. We can assume, by induction on $|A|$, that the proposition holds for the extension

$$
1 \longrightarrow A / Z(A) \longrightarrow \overline{\mathcal{T}} \stackrel{\tau}{\longrightarrow} \mathcal{T}_{\mathcal{H}}(G) \longrightarrow 1
$$

Thus there is an extension $\bar{G}$ of $G$ by $A / Z(A)$, a Sylow subgroup $\bar{S} \in \operatorname{Syl}_{p}(\bar{G})$, and a set $\overline{\mathcal{H}}$ of subgroups of $\bar{S}$, such that $\overline{\mathcal{T}} \cong \mathcal{T}_{\overline{\mathcal{H}}}(\bar{G})$.

Consider the following diagram:

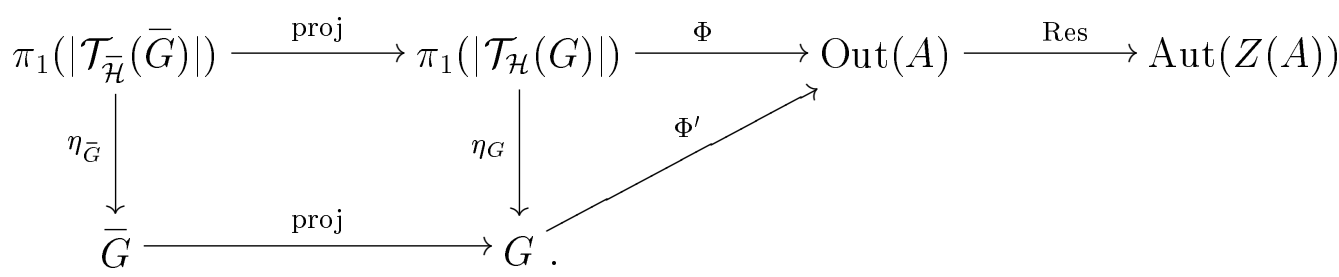

The triangle commutes by assumption, and the square by the naturality of $\eta_{G}$. The action of $\pi_{1}(|\overline{\mathcal{T}}|)$ on $Z(A)$ defined by the extension

$$
1 \longrightarrow Z(A) \longrightarrow \widetilde{\tau} \stackrel{\tau}{\longrightarrow} \mathcal{T}_{\overline{\mathcal{H}}}(\bar{G}) \longrightarrow 1
$$

is the composite of the upper row in (1) (see the definition of the action in Lemma 5.7), and it thus factors through $\bar{G}$ by the commutativity of (1). So by the abelian case of 
the proposition, applied to (2), there is an extension $\widetilde{G}$ of $\bar{G}$ by $Z(A)$ such that $\widetilde{\mathcal{T}}$ is a fully subcategory of $\mathcal{T}_{\widetilde{S}}(\widetilde{G})$. Also, $\widetilde{G}$ is an extension of $G$ by $A$, since $\mathcal{T}_{\mathcal{H}}(G) \cong \widetilde{\mathcal{T}} / A$.

Thus, in order to find an exotic extension, we must find an admissible action of some $\mathcal{T}_{\mathcal{H}}(G)$ on a $p$-group $A$ which does not factor through $G$. The following example was suggested to us by Albert Ruiz; it is just the simplest of a large family of such examples which he has constructed in $[\mathrm{Rz}]$. The example is stated in terms of the linking system of $G$, but it also holds if $\mathcal{L}_{S}^{c}(G)$ is replaced by the transporter system $\mathcal{T}_{S}^{c}(G)$ with the same objects.

Example 6.2. Fix a prime $p \geq 5$. Let $q$ be another prime, let e be the order of $q$ in $\mathbb{F}_{p}^{\times}($thus el$(p-1))$, and assume $e>2$. Set $G=G L_{e p}(q)$, fix $S \in \operatorname{Syl}_{p}(G)$, and set $\mathcal{F}=\mathcal{F}_{S}(G)$ and $\mathcal{L}=\mathcal{L}_{S}^{c}(G)$. Let $A$ be cyclic of order $p$. Then there is a homomorphism

$$
\Phi: \pi_{1}(|\mathcal{L}|)=\pi_{1}\left(\left|\mathcal{L}_{S}^{c}(G)\right|\right) \longrightarrow \operatorname{Aut}(A),
$$

where $|\operatorname{Im}(\Phi)|=e$, which does not factor through $G$. Also, there is an admissible extension

$$
1 \longrightarrow A \longrightarrow \widetilde{\mathcal{L}} \longrightarrow \mathcal{L}_{S}^{c}(G) \longrightarrow 1,
$$

where $\widetilde{\mathcal{L}}$ is a linking system associated to a saturated fusion system $\widetilde{\mathcal{F}}$ which is not the fusion system of any finite group.

Proof. Set $V=\left(\mathbb{F}_{q^{e}}\right)^{p}$ (with the canonical basis). Fix an $\mathbb{F}_{q^{-}}$basis for $\mathbb{F}_{q^{e}}$, which also yields an $\mathbb{F}_{q^{-}}$-basis for $V$. This allows us to identify $G L_{p}\left(q^{e}\right)=\operatorname{Aut}_{\mathbb{F}_{q}}(V)$ as a subgroup of $G L_{p e}(q)=\operatorname{Aut}_{\mathbb{F}_{q}}(V)$.

Let $\Gamma \leq \mathbb{F}_{p}^{\times}$be the subgroup of order $e$ generated by $q$. We identify this with $\operatorname{Gal}\left(\mathbb{F}_{q^{e}} / \mathbb{F}_{q}\right)$ (field automorphisms) by identifying $q \in \mathbb{F}_{p}^{\times}$with the automorphism $x \mapsto$ $x^{q}$. Set $H=\left(\mathbb{F}_{q^{e}}\right)^{\times} \rtimes \Gamma$, regarded as a subgroup of $G L_{e}(q)=\operatorname{Aut}_{\mathbb{F}_{q}}\left(\mathbb{F}_{q^{e}}\right)$ (vector space automorphisms). This defines an embedding of $H \imath \Sigma_{p}=H^{p} \rtimes \Sigma_{p}$ into $G L_{e p}(q)$.

Now,

$$
|G|=\prod_{i=0}^{e p-1}\left(q^{e p}-q^{i}\right)=q^{e p(e p-1) / 2} \cdot \prod_{i=1}^{e p}\left(q^{i}-1\right),
$$

where $p \mid\left(q^{i}-1\right)$ only when $e \mid i$, and $\left(q^{e i}-1\right) /\left(q^{e}-1\right) \equiv i(\bmod p)$. We leave it as an exercise to show that $\left(q^{e p}-1\right) /\left(q^{e}-1\right)$ is divisible by $p$ but not by $p^{2}$. Thus if $p^{\ell}$ is the largest power of $p$ dividing $q^{e}-1$, then $p^{p \ell+1}$ is the largest power of $p$ dividing $|G|$ and $\left|H<\Sigma_{p}\right|$; and this proves that $H<\Sigma_{p}$ contains a Sylow $p$-subgroup of $G$.

Fix $\Delta \in \operatorname{Syl}_{p}\left(\left(\mathbb{F}_{q^{e}}\right)^{\times}\right)$, a cyclic group of order $p^{\ell}$, and set $S=\Delta \curlywedge C_{p} \in \operatorname{Syl}_{p}\left(H \succ \Sigma_{p}\right) \subseteq$ $\operatorname{Syl}_{p}(G)$. Set $T=\Delta^{p} \leq S$ : the set of elements of $S$ which are diagonal in $G L_{p}\left(q^{e}\right)$.

We now want to define a homomorphism $\Psi^{\prime}$ from $\pi_{1}(|\mathcal{F}|)$ onto $\Gamma$, for which the kernel fusion system (in the sense of Proposition 4.1) is exotic. The existence of such a homomorphism is shown in $[\mathrm{Rz}]$, but we give a more explicit construction here.

Let $V \cong\left(\mathbb{F}_{q}\right)^{e p}$ be the vector space upon which $G$ acts. Consider the subset

$$
X=\left\{g \in S|| g \mid=p, \operatorname{Ker}\left(g-\operatorname{Id}_{V}\right)=0\right\} ;
$$

i.e., the set of elements of $S$ of order $p$ whose action on $V$ does not have 1 as eigenvalue. Every element of $S \backslash T$ acts on $V$ with characteristic polynomial $\left(X^{p}-1\right)^{e}$, which has 1 as root, and thus $X \subseteq T$. So if we fix $z \in\left(\mathbb{F}_{q^{e}}\right)^{\times}$of order $p$, and again identify $G L_{p}\left(q^{e}\right) \leq G L_{e p}(q)\left(\right.$ and $\left.\left(\mathbb{F}_{q^{e}}\right)^{p} \cong\left(\mathbb{F}_{q}\right)^{p e}\right)$, then

$$
X=\left\{\operatorname{diag}\left(z^{i_{1}}, \ldots, z^{i_{p}}\right) \mid i_{1}, \ldots, i_{p} \in \mathbb{F}_{p}^{\times}\right\} .
$$


By definition of $X$, any morphism in $\mathcal{F}=\mathcal{F}_{S}(G)$ sends elements of $X$ to elements of $X$.

Now, $\operatorname{Aut}_{\mathcal{F}}(T)=\operatorname{Aut}_{G}\left(\Delta^{p}\right) \cong \Gamma \imath \Sigma_{p}$ : the subgroup generated by the field automorphisms $\Gamma=\operatorname{Aut}\left(\mathbb{F}_{q^{e}}\right) \leq \operatorname{Aut}(\Delta)$, and by all permutations of the $p$ factors. Define $\Psi: \operatorname{Aut}_{\mathcal{F}}(T) \longrightarrow \Gamma$ to be the composite

$$
\Psi: \operatorname{Aut}_{\mathcal{F}}(T) \cong \Gamma^{p} \rtimes \Sigma_{p} \longrightarrow \Gamma \times \Sigma_{p} \stackrel{\mathrm{pr}_{1}}{\longrightarrow \Gamma} \text {. }
$$

Thus for all $\alpha \in \operatorname{Aut}_{\mathcal{F}}(T)$, if $\alpha(\operatorname{diag}(z, z, \ldots, z))=\operatorname{diag}\left(z^{i_{1}}, \ldots, z^{i_{p}}\right)$, then $\Psi(\alpha)=$ $i_{1} \cdots i_{p} \in \Gamma \leq \mathbb{F}_{p}^{\times}$. So $\Psi(\alpha)$ depends only on $\left.\alpha\right|_{Z(S) \cap X}$.

For each pair of subgroups $P, Q \leq S$ which are $p$-centric in $G$, define

$$
\Phi_{P, Q}^{\prime}: \operatorname{Hom}_{\mathcal{F}}(P, Q) \longrightarrow \Gamma
$$

as follows. For $\varphi \in \operatorname{Hom}_{\mathcal{F}}(P, Q),\left.\varphi\right|_{\langle P \cap X\rangle}$ extends to some $\bar{\varphi} \in \operatorname{Aut}_{\mathcal{F}}(T)$ by axiom (II) (and since $T$ is the unique abelian subgroup of index $p$ in $S$ ), and we set $\Phi_{P, Q}^{\prime}(\varphi)=$ $\Psi(\bar{\varphi})$. Since $\Psi(\bar{\varphi})$ depends only on $\left.\bar{\varphi}\right|_{Z(S) \cap X}$ and $P \geq Z(S)$, this is independent of the choice of extension $\bar{\varphi}$. If $\psi \in \operatorname{Hom}_{\mathcal{F}}(Q, R)$ is another morphism in $\mathcal{F}^{c}$, and $\bar{\psi} \in \operatorname{Aut}_{\mathcal{F}}(T)$ is an extension of $\left.\psi\right|_{\langle Q \cap X\rangle}$, then $\bar{\psi} \circ \bar{\varphi}$ is an extension of $\left.(\psi \circ \varphi)\right|_{\langle P \cap X\rangle}$ (since $\varphi(P \cap X) \subseteq Q \cap X)$ and thus $\Phi_{P, R}^{\prime}(\psi \circ \varphi)=\Phi_{Q, R}^{\prime}(\psi) \cdot \Phi_{P, Q}^{\prime}(\varphi)$.

We have thus defined a map $\Phi^{\prime}$ from $\operatorname{Mor}\left(\mathcal{F}^{c}\right)$ to $\Gamma$ which sends composites to products and sends inclusions to the identity. So by Proposition A.3(a), $\Phi^{\prime}$ can be identified with a homomorphism from $\pi_{1}\left(\left|\mathcal{F}^{c}\right|\right)$ to $\Gamma$. Set

$$
\Phi=\Phi^{\prime} \circ \pi_{1}(|\pi|): \pi_{1}(|\mathcal{L}|) \longrightarrow \Gamma \text {. }
$$

Let $(\widetilde{S}, \widetilde{\mathcal{F}}, \widetilde{\mathcal{L}})$ be the semidirect product extension of $(S, \mathcal{F}, \mathcal{L})$ by $A$ induced by $\Phi$; i.e., the extension which corresponds to the zero element in $H^{2}(|\mathcal{T}| ; A)$. In the notation of Theorem 5.11, $S_{1}=S$, and so the extension is admissible. Hence $\widetilde{\mathcal{F}}$ is a saturated fusion system by Theorem $5.11(\mathrm{~b})$. Also, since $\mathcal{L}=\mathcal{L}_{S}^{c}(G)$ is a linking system, $E(P)$ is a $p$-group for all $P \in \mathrm{Ob}(\mathcal{L})$, and thus $\widetilde{\mathcal{L}}$ is a linking system by Theorem $5.11(\mathrm{c})$.

Let $\mathcal{L}_{1} \subseteq \mathcal{L}$ and $\mathcal{F}_{1} \subseteq \mathcal{F}$ be the "kernel" linking and fusion systems:

$$
\operatorname{Mor}\left(\mathcal{L}_{1}\right)=\{\varphi \in \operatorname{Mor}(\mathcal{L}) \mid \Phi(\varphi)=1\} \quad \text { and } \quad \operatorname{Mor}\left(\mathcal{F}_{1}\right)=\left\{\varphi \in \operatorname{Mor}(\mathcal{F}) \mid \Phi^{\prime}(\varphi)=1\right\} \text {. }
$$

Thus by Proposition 4.1, $\mathcal{F}_{1}$ is a saturated fusion system over $S$ and $\mathcal{L}_{1}$ is a linking system associated to $\mathcal{F}_{1}$. By construction, $\operatorname{Aut}_{\mathcal{F}_{1}}(T) \cong \Gamma^{p-1} \rtimes \Sigma_{p}$. So by [BLO2, Example 9.4] (or more directly by [BLO2, Proposition 9.5]), $\mathcal{F}_{1}$ is not the fusion system of any finite group. (This is where we need to assume that $e>2$, and hence that $p \geq 5$.) Hence by Theorem $5.11(\mathrm{~d}), \widetilde{\mathcal{F}}$ is not the fusion system of any finite group.

We next make some cohomology computations, to illustrate the use of Lemma 5.12 and Corollary 5.13, and to show that the extension in Example 6.2 is the only one (up to isomorphism) with that action on the kernel.

Lemma 6.3. Let $G=G L_{e p}(q), S \in \operatorname{Syl}_{p}(G), A \cong C_{p}$, and $\pi_{1}\left(\left|\mathcal{L}_{S}^{c}(G)\right|\right) \stackrel{\Phi}{\longrightarrow} \operatorname{Aut}(A)$ be as in Example 6.2, set $\mathcal{F}=\mathcal{F}_{S}(G)$ and $\mathcal{L}=\mathcal{L}_{S}^{c}(G)$, and let $\mathcal{F}_{1} \subseteq \mathcal{F}$ and $\mathcal{L}_{1} \subseteq \mathcal{L}$ be the kernel subsystems. Then for $i=1,2, H^{i}(|\mathcal{L}| ; A) \cong H^{i}\left(\left|\mathcal{L}_{1}\right| ; \mathbb{F}_{p}\right)=0$.

Proof. Set $H=\left(\mathbb{F}_{q^{e}}^{\times} \rtimes C_{e}\right) \backslash \Sigma_{p}$, regarded as a subgroup of $G$ which contains $S$ via the embedding $\left(\mathbb{F}_{q^{e}}^{\times} \rtimes C_{e}\right) \leq G L_{e}(q)$. Let $\Psi$ denote the composite

$$
\Psi: H=\left(\mathbb{F}_{q^{e}}^{\times} \rtimes C_{e}\right) \imath \Sigma_{p} \longrightarrow C_{e} \imath \Sigma_{p} \longrightarrow C_{e} \times \Sigma_{p} \stackrel{\mathrm{pr}_{1}}{\longrightarrow} C_{e} \leq \mathbb{F}_{p}^{\times},
$$


where the second homomorphism takes the product of the $p$ coordinates in $C_{e}$, and set $H_{1}=\operatorname{Ker}(\Psi)$. Set

$$
\bar{H}_{1}=H_{1} \cap\left(\mathbb{F}_{q^{e}}^{\times} \rtimes C_{e}\right)^{p} \cong\left(\mathbb{F}_{q^{e}}^{\times}\right)^{p} \rtimes\left(C_{e}\right)^{p-1}
$$

Then

$$
H^{i}\left(\bar{H}_{1} ; \mathbb{F}_{p}\right)=H^{i}\left(\left(\mathbb{F}_{q^{e}}^{\times}\right)^{p} ; \mathbb{F}_{p}\right)^{\left(C_{e}\right)^{p-1}}
$$

vanishes for $i=1,2$, and similarly for

$$
H^{i}\left(\Sigma_{p} ; \mathbb{F}_{p}\right) \stackrel{\text { mono }}{\longrightarrow} H^{i}\left(C_{p} \rtimes C_{p-1} ; \mathbb{F}_{p}\right) .
$$

So by the spectral sequence for the semidirect product, $H^{i}\left(H_{1} ; \mathbb{F}_{p}\right)=0$ for $i=1,2$.

By [BLO2, Theorem B], the inclusion $\mathcal{B}(S) \subseteq \mathcal{L}_{1}$ induces a monomorphism

$$
H^{*}\left(\left|\mathcal{L}_{1}\right| ; \mathbb{F}_{p}\right) \longrightarrow H^{*}\left(S ; \mathbb{F}_{p}\right),
$$

and this factors through $H^{*}\left(H_{1} ; \mathbb{F}_{p}\right)$. Hence $H^{i}\left(\left|\mathcal{L}_{1}\right| ; \mathbb{F}_{p}\right)=0$ for $i=1,2$. So by Corollary 5.13, if $\pi_{1}(|\mathcal{L}|)$ acts on $A$ via $\Phi$, then $H^{2}(|\mathcal{L}| ; A) \cong H^{2}(\Gamma ; A)=0$ (since $\Gamma$ has order prime to $p$ ).

By Lemma 6.3 and Proposition 5.8, it now follows that the only extension of $\mathcal{L}$ by $A$ (with the given action) is the "semidirect product" constructed in Example 6.2.

Example 6.2 is interesting as an example of an exotic fusion system with normal $p$ subgroup for which the quotient is the fusion system of a group. But it is "too simple" in some sense, and leaves a lot of questions as to what other sorts of extensions can occur. This motivates the following list of problems.

In all cases, in the following list, $1 \rightarrow A \rightarrow \widetilde{\mathcal{T}} \rightarrow \mathcal{T} \rightarrow 1$ is an extension of transporter systems, associated to fusion systems $\widetilde{\mathcal{F}}$ and $\mathcal{F}$ over $p$-groups $\widetilde{S}$ and $S$ (and where $A$ is abelian). We always let $\Phi$ denote the action of $\pi_{1}(|\mathcal{T}|)$ on $A$. The phrase "interesting extension" is intentionally left imprecise, but at least means an extension of transporter systems $1 \rightarrow A \rightarrow \widetilde{\mathcal{T}} \rightarrow \mathcal{T} \rightarrow 1$ which is not induced by an extension of finite groups.

(1) Find interesting extensions for which $\operatorname{Im}(\Phi)$ has order a multiple of $p$.

(2) Find interesting extensions where subgroups which are centric and radical in $\widetilde{\mathcal{F}}$ are not all sent to subgroups which are quasicentric in $\mathcal{F}$.

(3) Find an extension where $\mathcal{F}$ and $\mathcal{F}_{1}$ (the kernel subsystem) are both fusion systems of groups, but where $\widetilde{\mathcal{F}}$ is exotic. Essentially, this means finding $\mathcal{F}_{1} \triangleleft \mathcal{F}$ which are fusion systems of groups, but not fusion systems of a pair of groups $G_{1}$ and $G$ such that $G_{1} \triangleleft G$ with appropriate quotient.

We refer to [BCGLO1, Definition 3.1] for the definition of an $\mathcal{F}$-quasicentric subgroup. When $\mathcal{F}=\mathcal{F}_{S}(G)$ for a finite group $G$, then a subgroup $P \leq S$ is $\mathcal{F}$-quasicentric if and only if $C_{G}(P)$ has a normal subgroup of order prime to $p$ and of $p$-power index. Question (2) is motivated by [BCGLO1, Theorem B], which says that for any $p$-local finite group $(S, \mathcal{F}, \mathcal{L})$, there is a linking system $\mathcal{L}^{q} \supseteq \mathcal{L}$ for which $\operatorname{Ob}\left(\mathcal{L}^{q}\right)$ is the set of all $\mathcal{F}$-quasicentric subgroups of $S$, and that for any full subcategory $\mathcal{L}_{0} \subseteq \mathcal{L}^{q}$ which contains all $\mathcal{F}$-centric $\mathcal{F}$-radical subgroups of $S,\left|\mathcal{L}_{0}\right|,|\mathcal{L}|$, and $\left|\mathcal{L}^{q}\right|$ all have the same homotopy type.

In the remaining part of this section, we describe some constructions which are simple modifications of the one in Example 6.2, and which show that problems (1) and (2) have solutions if one keeps the requirements for "interesting extensions" to a 
minimum. What this really shows is that we want more restrictive conditions on what makes them "interesting".

Let $p, q$, and $e$ be as in Example 6.2: $p$ is a prime with $p \geq 5, q$ is a prime power with $p \nmid q$, and $e \geq 3$ is the order of $q$ in $\mathbb{F}_{p}^{\times}$. Set $H=G L_{p e}\left(q^{p}\right)$, and let $G=H \rtimes C_{p}$ where $C_{p}$ acts via the field automorphism of order $p$ on $\mathbb{F}_{q^{p}}$. Fix $S_{0} \in \operatorname{Syl}_{p}(H)$ and $S=S_{0} \rtimes C_{p} \in \operatorname{Syl}_{p}(G)$, and set $\mathcal{L}_{0}=\mathcal{L}_{S_{0}}^{c}(H)$. Set

$$
\mathcal{H}=\left\{P \leq S \mid Z(P) \cap H \in \operatorname{Syl}_{p}\left(C_{H}(P)\right)\right\} .
$$

For each $P \in \mathcal{H}, C_{H}(P)=(Z(P) \cap H) \times C_{H}^{\prime}(P)$ where $C_{H}^{\prime}(P)$ is the subgroup of elements of order prime to $p$ in $C_{H}(P)$, and we set

$$
\mathcal{T}=\mathcal{T}_{\mathcal{H}}(G) / C_{H}^{\prime} .
$$

This is a transporter system by Proposition 3.13. Also, $\mathcal{L}_{0}$ is the kernel linking system of the homomorphism from $\pi_{1}(|\mathcal{T}|)$ to $C_{p}$ induced by the surjection of $\pi_{1}\left(\left|\mathcal{T}_{\mathcal{H}}(G)\right|\right)$ onto $G / H$. Hence the homomorphism of Example 6.2 extends to a homomorphism

$$
\widehat{\Phi}: \pi_{1}(|\mathcal{T}|) \longrightarrow \Gamma \times C_{p} \leq \operatorname{Aut}(A), \quad\left(A \cong C_{p^{2}}\right)
$$

and the homomorphism is admissible by construction of $\mathcal{T}$. The kernel linking system of $\widehat{\Phi}$ is the same as the kernel system for $\pi_{1}\left(\left|\mathcal{L}_{0}\right|\right) \stackrel{\Phi_{0}}{\longrightarrow} \Gamma$, which was shown to be exotic in Example 6.2. So by Theorem 5.11, for any extension

$$
1 \longrightarrow A \longrightarrow \widetilde{\mathcal{L}} \longrightarrow \mathcal{T} \longrightarrow 1
$$

which realizes this action of $\mathcal{T}$ on $A, \widetilde{\mathcal{L}}$ is a linking system associated to an exotic fusion system. Using Lemmas 5.12 and 6.3 , we see that $H^{2}(|\mathcal{L}| ; A) \cong H^{2}\left(C_{e} \times C_{p} ; A\right)=0$, so there is a unique extension of this type.

A similar, but slightly more complicated example can be constructed by setting $H=G L_{p e}(q)^{p}, G=G L_{p e}(q) \imath \Sigma_{p}=H \rtimes \Sigma_{p}, S \in \operatorname{Syl}_{p}(G)$, and $\mathcal{T}=\mathcal{T}_{\mathcal{H}}(G) / C_{H}^{\prime}$ for an appropriate class $\mathcal{H}$ of subgroups of $S$. One can then define $\Phi$ from $\pi_{1}(|\mathcal{T}|)$ to $\Gamma<\Sigma_{p}$, where $\Gamma$ again is cyclic of order $e$, and look at the induced extensions of $A=\left(C_{p}\right)^{p}$ by $\mathcal{T}$.

As yet another example, let $p, q$, and $e$ be as in Example 6.2, set $G_{1}=G L_{p e}(q)$, and set $G_{2}=S L_{3}(p)$. Fix $S_{i} \in \operatorname{Syl}_{p}\left(G_{i}\right)$, and set $G=G_{1} \times G_{2}$ and $S=S_{1} \times S_{2} \in \operatorname{Syl}_{p}(G)$. Set

$$
\mathcal{H}=\left\{P \leq S \mid Z(P) \cap G_{1} \in \operatorname{Syl}_{p}\left(C_{G_{1}}(P)\right)\right\} .
$$

For each $P \in \mathcal{H}, C_{G_{1}}(P)=\left(Z(P) \cap G_{1}\right) \times C_{G_{1}}^{\prime}(P)$, where $C_{G_{1}}^{\prime}(P)$ consists of all elements in $C_{G_{1}}(P)$ of order prime to $p$. Set $\mathcal{T}=\mathcal{T}_{\mathcal{H}}(G) / C_{G_{1}}^{\prime}$, a transporter system by Proposition 3.13. Define

$$
\Phi: \pi_{1}(|\mathcal{T}|) \longrightarrow \pi_{1}\left(\left|\mathcal{L}_{S_{1}}^{c}\left(G_{1}\right)\right|\right) \times \pi_{1}\left(\left|\mathcal{T}_{S_{2}}\left(G_{2}\right)\right|\right) \longrightarrow \operatorname{Aut}\left(C_{p}\right) \times \operatorname{Aut}\left(C_{p}^{3}\right),
$$

where the first homomorphism is induced by the two projections, and the second is the product of the homomorphism of Example 6.2 with the canonical action of $G_{2}=S L_{3}(p)$ on $C_{p}^{3}$. Set $A=C_{p} \times C_{p}^{3}$, let

$$
1 \longrightarrow A \longrightarrow \widetilde{\mathcal{L}} \longrightarrow \mathcal{T} \longrightarrow 1
$$

be any extension which realizes $\Phi$, and let $\widetilde{S}$ and $\widetilde{\mathcal{F}}$ be the associated $p$-group and fusion system. By Theorem 5.11 again, $\widetilde{\mathcal{F}}$ is a saturated fusion system over $\widetilde{S}$, but $\widetilde{\mathcal{F}}$ is not the fusion system of any finite group, since if it were the fusion system of some 
$\widetilde{G}$, then $C_{\widetilde{G}}(A) / A$ would have as fusion system the subsystem of index $e$ in $\mathcal{F}_{S_{1}}\left(G_{1}\right)$. Also, for each $P \in \mathrm{Ob}(\mathcal{T})$,

$$
\operatorname{Ker}\left[\Phi: C_{G}(P) / C_{G_{1}}^{\prime}(P) \longrightarrow \operatorname{Aut}(A)\right] \leq C_{G_{1}}(P) / C_{G_{1}}^{\prime}(P)
$$

is a $p$-group, and so the restriction of $\widetilde{\mathcal{L}}$ to $\widetilde{\mathcal{F}}$-centric subgroups is a linking system by Theorem 5.11(c).

If we now take $P=S_{1} \times 1 \in \mathcal{H}$, and let $\widetilde{P}$ be such that $\widetilde{P} / A=P$, then $\widetilde{P}$ is centric and radical in $\widetilde{\mathcal{F}}$. However, $P$ is not quasicentric in $\mathcal{F}_{S}(G)$, since its centralizer fusion system involves the fusion system of $G_{2}$.

\section{Appendix A. General BaCkground on CATEgories And higher limits}

We begin with a description of the "bar resolution" for computing higher derived functors of inverse limits of diagrams of abelian groups. For any small category $\mathcal{C}$, $\mathcal{C}$-mod denotes the category of functors $\mathcal{C}^{\text {op }} \longrightarrow A b$.

Lemma A.1. Let $\mathcal{C}$ be any small category, and let $F: \mathcal{C}^{\mathrm{op}} \longrightarrow$ Ab be any functor. Let $\bar{C}^{*}(\mathcal{C} ; F)$ be the normalized chain complex for $F$ :

$$
\bar{C}^{n}(\mathcal{C} ; F)=\prod_{c_{0} \rightarrow \cdots \rightarrow c_{n}} F\left(c_{0}\right),
$$

where the product is taken over all composable sequences of nonidentity morphisms. For $\xi \in \bar{C}^{n}(\mathcal{C} ; F)$, define

$$
\begin{aligned}
d(\xi)\left(c_{0} \stackrel{\varphi}{\rightarrow} c_{1} \rightarrow \cdots \rightarrow c_{n+1}\right)=F(\varphi)\left(\xi \left(c_{1} \rightarrow\right.\right. & \left.\left.\rightarrow c_{n+1}\right)\right)+ \\
& \sum_{i=1}^{n+1}(-1)^{i} \xi\left(c_{0} \rightarrow \cdots \widehat{c_{i}} \cdots \rightarrow c_{n+1}\right),
\end{aligned}
$$

where we set $\xi\left(c \rightarrow \cdots \rightarrow c^{\prime}\right)=0$ for any sequence containing an identity morphism. Then ${\underset{\mathcal{C}}{\lim }}^{*}(F) \cong H^{*}\left(\bar{C}^{*}(\mathcal{C} ; F), d\right)$.

Proof. The proof is the same as those in [GZ, Appendix II, Proposition 3.3] or [O1, Lemma 2] (where the result is shown for the unnormalized chain complex). We sketch the argument here. Let $\mathfrak{P}_{-1}$ be the constant functor $\underline{\mathbb{Z}}$. For each $n \geq 0$, define $\mathfrak{P}_{n}: \mathcal{C}^{\mathrm{op}} \longrightarrow \mathrm{Ab}$ by letting $\mathfrak{P}_{n}(c)$ be the free abelian group with basis the set of all sequences $c \stackrel{\varphi}{\rightarrow} c_{0} \stackrel{\varphi_{1}}{\longrightarrow} \cdots \stackrel{\varphi_{n}}{\longrightarrow} c_{n}$, modulo the subgroup generated by those sequences for which some $\varphi_{i}$ for $i \geq 1$ is an identity morphism. Also, for $\psi \in \operatorname{Mor}(\mathcal{C}), \mathfrak{P}_{n}(\psi)$ is defined by composing the first morphism with $\psi$. For all $n \geq 0$ and all $F$ in $\mathcal{C}$-mod, $\operatorname{Mor}_{\mathcal{C} \text {-mod }}\left(\mathfrak{P}_{n}, F\right) \cong \bar{C}^{n}(\mathcal{C} ; F)$. Thus $\mathfrak{P}_{n}$ is projective in $\mathcal{C}$-mod for $n \geq 0$. Boundary morphisms $\partial: \mathfrak{P}_{n} \longrightarrow \mathfrak{P}_{n-1}$ are defined by setting

$$
\partial\left(\left[c \rightarrow c_{0} \rightarrow \cdots \rightarrow c_{n}\right]\right)=\sum_{i=0}^{n}(-1)^{i}\left[c \rightarrow c_{0} \rightarrow \cdots \widehat{c_{i}} \cdots \rightarrow c_{n}\right] .
$$

This is seen to be exact (hence a projective resolution of $\underline{\mathbb{Z}}$ ) via the splitting homomorphisms $\mathfrak{P}_{n}(c) \longrightarrow \mathfrak{P}_{n+1}(c)$ which send $\left[c \rightarrow c_{0} \rightarrow \cdots \rightarrow c_{n}\right]$ to $\left[c \stackrel{\text { Id }}{\rightarrow} c \rightarrow c_{0} \rightarrow \cdots \rightarrow\right.$ 
$\left.c_{n}\right]$. Since ${\underset{\mathcal{C}}{\lim _{\mathcal{C}}}}^{0}(F) \cong \operatorname{Mor}_{\mathcal{C}-\bmod }(\underline{\mathbb{Z}}, F)$,

$$
{\underset{\mathcal{C}}{\lim _{\mathcal{C}}}}^{*}(F) \cong \operatorname{Ext}_{\mathcal{C}-\bmod }^{*}(\underline{\mathbb{Z}}, F) \cong H^{*}\left(\operatorname{Mor}_{\mathcal{C}-\bmod }\left(\mathfrak{P}_{*}, F\right), \partial^{*}\right) \cong H^{*}\left(\bar{C}^{*}(\mathcal{C} ; F), d\right) .
$$

For any finite group $G$, we let $\mathcal{O}_{p}(G)$ be the $p$-subgroup orbit category of $G$ : the category whose objects are the $p$-subgroups of $G$, and where

$$
\operatorname{Mor}_{\mathcal{O}_{p}(G)}(P, Q)=Q \backslash N_{G}(P, Q) \cong \operatorname{map}_{G}(G / P, G / Q) .
$$

Here, $N_{G}(P, Q)$ is the transporter set:

$$
N_{G}(P, Q)=\left\{g \in G \mid g P g^{-1} \leq Q\right\} .
$$

If $S \in \operatorname{Syl}_{p}(G)$, then $\mathcal{O}_{S}(G) \subseteq \mathcal{O}_{p}(G)$ denotes the full subcategory whose objects are the subgroups of $S$. These two categories are clearly equivalent (every object in $\mathcal{O}_{p}(G)$ is isomorphic to an object in $\mathcal{O}_{S}(G)$ ). Note that $\mathcal{O}_{S}(G)$ is not the same as the orbit category of the fusion system $\mathcal{F}_{S}(G)$.

For any $\mathbb{Z}[G]$-module $M$, we define

$$
\Lambda^{*}(G ; M)=\underset{\mathcal{O}_{p}(G)}{\lim ^{*}}\left(F_{M}\right)
$$

where $F_{M}: \mathcal{O}_{p}(G)^{\mathrm{op}} \longrightarrow \mathrm{Ab}$ is the functor $F_{M}(P)=0$ if $P \neq 1$ and $F_{M}(1)=M$. The role these groups play in computing higher limits of certain functors is illustrated by the following proposition, which is a special case of [BLO3, Proposition 4.3].

Proposition A.2. Fix a category $\mathcal{C}$, a finite group $G$, a Sylow subgroup $S \in \operatorname{Syl}_{p}(G)$, and a functor

$$
\alpha: \mathcal{O}_{S}(G) \longrightarrow \mathcal{C} \text {. }
$$

Set $c_{0}=\alpha(1)$. For each object $d$ in $\mathcal{C}$, we regard the set $\operatorname{Mor}_{\mathcal{C}}\left(c_{0}, d\right)$ as a $G$-set via $\alpha$ and composition. Assume that the following conditions hold:

(a) $\alpha$ sends $G=\operatorname{Aut}_{\mathcal{O}_{S}(G)}(1)$ bijectively to $\operatorname{End}_{\mathcal{C}}\left(c_{0}\right)$.

(b) For each $d \in \mathrm{Ob}(\mathcal{C})$ such that $d \neq c_{0}$, all isotropy subgroups of the $G$-action on $\operatorname{Mor}_{\mathcal{C}}\left(c_{0}, d\right)$ are nontrivial p-subgroups.

(c) For each $\xi \in \operatorname{Mor}\left(\mathcal{O}_{p}(G)\right), \alpha(\xi)$ is an epimorphism in the categorical sense: $\varphi$ 。 $\alpha(\xi)=\psi \circ \alpha(\xi)$ implies $\varphi=\psi$.

(d) For each $P \leq S$, each $d \in \mathrm{Ob}(\mathcal{C})$, and each $\varphi \in \operatorname{Mor}_{\mathcal{C}}\left(c_{0}, d\right)$ which is fixed by the $P$-action, there is some $\bar{\varphi} \in \operatorname{Mor}_{\mathcal{C}}(\alpha(P), d)$ such that $\varphi=\bar{\varphi} \circ \alpha\left(\operatorname{incl}_{1}^{P}\right)$.

Let

$$
\Phi: \mathcal{C}^{\mathrm{op}} \longrightarrow \mathrm{Ab}
$$

be any functor which vanishes except on the isomorphism class of $c_{0}$. Then the natural map

is an isomorphism.

$$
\lim _{\mathcal{C}} * \underset{\alpha^{*}}{\cong}{\underset{\mathcal{O}}{\stackrel{\lim _{S}(G)}{*}}}^{*}(\Phi \circ \alpha) \cong \Lambda^{*}\left(G ; \Phi\left(c_{0}\right)\right)
$$

We now look at fundamental groups of nerves of categories. The next proposition describes the relationship between an action of $\pi_{1}(|\mathcal{C}|)$ and an action of $\operatorname{Mor}(\mathcal{C})$.

For any group $\Gamma, \mathcal{B}(\Gamma)$ denotes the category with one object and with automorphism group $\Gamma$, and we identify $|\mathcal{B}(G)|=B G$. This notation will be used in several of the proofs in this section. 
Proposition A.3. Fix a small category $\mathcal{C}$, and an object $c_{0}$ in $\mathcal{C}$. Assume, for each $c \in \mathrm{Ob}(\mathcal{C})$, that $\operatorname{Mor}_{\mathcal{C}}\left(c, c_{0}\right) \neq \varnothing$, and fix some distinguished "inclusion" morphism $\iota_{c} \in \operatorname{Mor}_{\mathcal{C}}\left(c, c_{0}\right)$ (where $\left.\iota_{c_{0}}=\operatorname{Id}_{c_{0}}\right)$. For each morphism $\psi \in \operatorname{Mor}_{\mathcal{C}}(c, d)$, let $\theta(\psi) \in$ $\pi_{1}\left(|\mathcal{C}|, c_{0}\right)$ be the element represented by the loop $\iota_{d} \cdot \psi \cdot \iota_{c}{ }^{-1}$ (where paths are composed from right to left). Then the following hold:

(a) For any group $\Gamma$, and any function $F: \operatorname{Mor}(\mathcal{C}) \longrightarrow \Gamma$ such that $F\left(\iota_{c}\right)=1$ for all $c$ and $F(\psi \circ \varphi)=F(\psi) F(\varphi)$ when $\psi \circ \varphi$ is defined, there is a unique homomorphsm $\bar{F}: \pi_{1}\left(|\mathcal{C}|, c_{0}\right) \longrightarrow \Gamma$ such that $\bar{F}(\theta(\psi))=F(\psi)$ for all $\psi \in \operatorname{Mor}(\mathcal{C})$. In other words, $\pi_{1}\left(|\mathcal{C}|, c_{0}\right)$ is the free group on the morphisms in $\mathcal{C}$ modulo the relations given by composition and the $\iota_{c}$.

(b) Let $F: \mathcal{C} \longrightarrow \mathrm{Ab}$ be any functor which sends all morphisms in $\mathcal{C}$ to isomorphisms of groups. Set $A=F\left(c_{0}\right)$, and let $F^{\prime}$ be the functor which sends each object to $A$, and where $F^{\prime}(\psi)=F\left(\iota_{d}\right) \circ F(\psi) \circ F\left(\iota_{c}\right)^{-1}$ for $\psi \in \operatorname{Mor}_{\mathcal{C}}(c, d)$. Then $F^{\prime}$ is naturally isomorphic to F. Also,

$$
{\underset{\mathcal{C}}{\lim }}^{*}(F) \cong H^{*}(|\mathcal{C}| ; A)
$$

where the second group is cohomology twisted via the linear action of $\pi_{1}(|\mathcal{C}|)$ on $A$ induced by $F^{\prime}$.

Proof. (a) By elementary homotopy theory, any element of $\pi_{1}\left(|\mathcal{C}|, c_{0}\right)$ is represented by a loop which follows the edges of $|\mathcal{C}|$, and hence is a product of loops of the form $\theta(\varphi)$ for $\varphi \in \operatorname{Mor}(\mathcal{C})$. Thus $\pi_{1}(|\mathcal{C}|)=\langle\operatorname{Im}(\theta)\rangle$. This also proves the uniqueness of the homomorphism $\bar{F}$, and it remains to prove its existence.

Let $\widetilde{F}: \mathcal{C} \longrightarrow \mathcal{B}(\Gamma)$ be the functor which sends all objects of $\mathcal{C}$ to the unique object of $\mathcal{B}(\Gamma)$, and which sends morphisms via $F$. Set

$$
\bar{F}=\pi_{1}(|\widetilde{F}|): \pi_{1}\left(|\mathcal{C}|, c_{0}\right) \longrightarrow \Gamma \text {. }
$$

For each $\psi \in \operatorname{Mor}(\mathcal{C})$,

$$
\bar{F}(\theta(\psi))=\bar{F}\left(\iota_{d} \cdot \psi \cdot \iota_{c}{ }^{-1}\right)=F\left(\iota_{d}\right) \cdot F(\psi) \cdot F\left(\iota_{c}\right)^{-1}=F(\psi)
$$

since $F\left(\iota_{c}\right)=F\left(\iota_{d}\right)=1$ by assumption.

(b) The functor $F^{\prime}$ is naturally isomorphic to $F$ via the natural isomorphism which sends each $c \in \operatorname{Ob}(\mathcal{C})$ to $\iota_{c}$. The map from $\operatorname{Mor}(\mathcal{C})$ to $\operatorname{Aut}(A)$ defined by $F^{\prime}$ factors through $\pi_{1}(|\mathcal{C}|)$ by $\left(\right.$ a). The chain complex $\bar{C}^{*}\left(\mathcal{C} ; F^{\prime}\right)$ of Lemma A.1 is precisely the same as the chain complex $C^{*}(|\mathcal{C}| ; A)$ for cohomology with twisted coefficients, and thus

$$
{\underset{\iota}{\mathcal{C}}}^{\lim ^{*}}(F) \cong{\underset{\mathcal{C}}{\lim }}^{*}\left(F^{\prime}\right) \cong H^{*}(|\mathcal{C}| ; A) \text {. }
$$

The following proposition describes very general conditions for the nerve of a subcategory of $\mathcal{C}$ to have the homotopy type of a covering space of $|\mathcal{C}|$.

Proposition A.4. Fix a small category $\mathcal{C}$, a group $\Gamma$, and a surjective homomorphism $\bar{F}: \pi_{1}(|\mathcal{C}|) \longrightarrow \Gamma$. Set $F=\bar{F} \circ \theta: \operatorname{Mor}(\mathcal{C}) \longrightarrow \Gamma$, where $\theta$ is as in Proposition A.3. For each $H \leq \Gamma$, let $\mathcal{C}_{H} \subseteq \mathcal{C}$ be the subcategory with the same objects, where for all $\psi \in \operatorname{Mor}(\mathcal{C}), \psi \in \operatorname{Mor}\left(\mathcal{C}_{H}\right)$ if and only if $F(\psi) \in H$. Assume that

(1) for each $c \in \mathrm{Ob}(\mathcal{C})$ and each $g \in \Gamma$, there is an object $d \in \mathrm{Ob}(\mathcal{C})$ and an isomorphism $\psi \in \operatorname{Iso}_{\mathcal{C}}(c, d)$ such that $F(\psi)=g$. 
Then for each $H \leq \Gamma,\left|\mathcal{C}_{H}\right|$ is homotopy equivalent to the covering space of $|\mathcal{C}|$ whose fundamental group is $\bar{F}^{-1}(H)$.

Proof. For any set $X$ with left $\Gamma$-action, let $\mathcal{E}_{\Gamma}(X)$ be the category with object set $X$, and where

$$
\operatorname{Mor}_{\mathcal{E}_{\Gamma}(X)}(x, y)=\{g \in \Gamma \mid g x=y\} .
$$

Let $\widetilde{\mathcal{C}}_{H}$ be the pullback category in the following square:

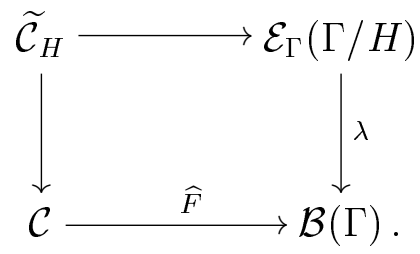

Here, $\widehat{F}$ and $\lambda$ send all objects to the unique object of $\mathcal{B}(\Gamma) ; \widehat{F}$ sends morphisms via $F$, and $\lambda$ sends $g \in \operatorname{Mor}_{\mathcal{E}_{\Gamma}(\Gamma / H)}(a H, b H)$ (if $\left.g a H=b H\right)$ to $g \in \operatorname{Mor}(\mathcal{B}(\Gamma)$ ). Thus $\operatorname{Ob}\left(\widetilde{\mathcal{C}}_{H}\right)=\operatorname{Ob}(\mathcal{C}) \times(\Gamma / H)$, and $\operatorname{Mor}\left(\widetilde{\mathcal{C}}_{H}\right)$ is the set of pairs of morphisms in $\mathcal{C}$ and $\mathcal{E}_{\Gamma}(\Gamma / H)$ which get sent to the same morphism in $\mathcal{B}(\Gamma)$. Then $\left|\widetilde{\mathcal{C}}_{H}\right|$ is a covering space of $|\mathcal{C}|$ by construction; and is the covering space with fundamental group $\bar{F}^{-1}(H)$ since $\pi_{1}\left(\left|\mathcal{E}_{\Gamma}(\Gamma / H)\right|\right)=H$.

The subcategory $\mathcal{C}_{H}$ can be identified with the full subcategory of $\widetilde{\mathcal{C}}_{H}$ whose objects are the pairs $(c, 1 H)$ for $c \in \mathrm{Ob}(\mathcal{C})$. Under this identification, each object in $\widetilde{\mathcal{C}}_{H}$ is isomorphic to an object in $\mathcal{C}_{H}$ by $(1)$, and thus $\left|\mathcal{C}_{H}\right| \simeq\left|\widetilde{\mathcal{C}}_{H}\right|$.

We now discuss extensions of categories. We want to look at the following very general situation, which occurs in several different contexts throughout the paper.

Definition A.5. Let $\tau: \widetilde{\mathcal{C}} \longrightarrow \mathcal{C}$ be a functor between categories which is a bijection on objects and surjective on morphism sets. For each $\widetilde{c} \in \mathrm{Ob}(\widetilde{\mathcal{C}})$, set

$$
K(\widetilde{c}) \stackrel{\text { def }}{=} \operatorname{Ker}\left[\tau_{\widetilde{c}, \widetilde{c}}: \operatorname{Aut}_{\widetilde{\mathcal{C}}}(\widetilde{c}) \longrightarrow \operatorname{Aut}_{\mathcal{C}}(\tau(\widetilde{c}))\right] .
$$

(a) We say that $\tau$ is source regular (or a source regular extension of $\mathcal{C}$ by $K$ ) if for all $\widetilde{c}, \widetilde{d} \in \mathrm{Ob}(\widetilde{\mathcal{C}}), K(\widetilde{c})$ acts freely on $\operatorname{Mor}_{\widetilde{\mathcal{C}}}(\widetilde{c}, \widetilde{d})$ and $\tau_{\widetilde{c}, \widetilde{d}}$ is the orbit map for this action.

(b) We say that $\tau$ is target regular (or a target regular extension of $\mathcal{C}$ by $K$ ) if for all $\widetilde{c}, \widetilde{d} \in \operatorname{Ob}(\widetilde{\mathcal{C}}), K(\widetilde{d})$ acts freely on $\operatorname{Mor}_{\widetilde{\mathcal{C}}}(\widetilde{c}, \widetilde{d})$ and $\tau_{\widetilde{c}, \widetilde{d}}$ is the orbit map for this action.

The next three lemmas list some of the elementary properties of these classes of functors. For simplicity in notation throughout the rest of this section, when working with functors $\widetilde{\mathcal{C}} \longrightarrow \mathcal{C}$ which are source or target regular, we identify each $c \in \mathrm{Ob}(\widetilde{\mathcal{C}})$ with $\tau(c) \in \mathrm{Ob}(\mathcal{C})$.

Lemma A.6. If $\tau: \widetilde{\mathcal{C}} \longrightarrow \mathcal{C}$ is either source or target regular, then a morphism in $\widetilde{\mathcal{C}}$ is an isomorphism if and only if its image in $\mathcal{C}$ is an isomorphism. In particular, if all endomorphisms in $\mathcal{C}$ are automorphisms, then the same is true of $\widetilde{\mathcal{C}}$.

Proof. Clearly, $\tau$ sends isomorphisms to isomorphisms, and we need only prove the converse. Assume $\widetilde{\psi} \in \operatorname{Mor}_{\widetilde{\mathcal{C}}}(c, d)$ is such that $\tau_{c, d}(\widetilde{\psi})$ is an isomorphism, and choose $\widetilde{\varphi} \in \operatorname{Mor}_{\widetilde{\mathcal{C}}}(d, c)$ such that $\tau(\widetilde{\varphi})=\tau(\widetilde{\psi})^{-1}$. Since $\tau_{c, c}$ is the orbit map for a free action of 
$K(c)$, and $\tau_{c, c}(\widetilde{\varphi} \circ \widetilde{\psi})=\tau_{c, c}\left(\operatorname{Id}_{c}\right), \widetilde{\varphi} \circ \widetilde{\psi} \in K(c)$, and hence is an automorphism. Similarly, $\widetilde{\psi} \circ \widetilde{\varphi} \in K(d) \leq \operatorname{Aut}_{\widetilde{\mathcal{C}}}(d)$, and thus $\widetilde{\psi}$ and $\widetilde{\varphi}$ are both isomorphisms.

Let $\mathrm{Gps}$ be the category of groups with homomorphisms. Let $\mathrm{Gps}_{*}$ be the category with the same objects, and where

$$
\operatorname{Mor}_{\mathrm{Gps}_{*}}(G, H)=\operatorname{Rep}(G, H) \stackrel{\text { def }}{=} \operatorname{Inn}(H) \backslash \operatorname{Hom}(G, H) .
$$

Lemma A.7. The following hold for any source regular functor $\tau: \widetilde{\mathcal{C}} \longrightarrow \mathcal{C}$.

(a) For any sequence $c_{0} \stackrel{\varphi}{\rightarrow} c_{1} \stackrel{\psi}{\rightarrow} c_{2}$ of morphisms in $\mathcal{C}$, and any pair of morphisms $\widetilde{\psi} \in \operatorname{Mor}_{\widetilde{\mathcal{C}}}\left(c_{1}, c_{2}\right)$ and $\widetilde{\psi \varphi} \in \operatorname{Mor}_{\widetilde{\mathcal{C}}}\left(c_{0}, c_{2}\right)$ such that $\tau(\widetilde{\psi})=\psi$ and $\tau(\widetilde{\psi \varphi})=\psi \varphi$, there is a unique morphism $\widetilde{\varphi} \in \operatorname{Mor}_{\widetilde{\mathcal{C}}}\left(c_{0}, c_{1}\right)$ such that $\tau(\widetilde{\varphi})=\varphi$ and $\widetilde{\psi} \circ \widetilde{\varphi}=\widetilde{\psi \varphi}$.

(b) For each $\widetilde{\psi} \in \operatorname{Mor}(\widetilde{\mathcal{C}}), \widetilde{\psi}$ is a monomorphism in $\widetilde{\mathcal{C}}$ in the categorical sense (i.e., $\widetilde{\psi} \circ \widetilde{\varphi}_{1}=\widetilde{\psi} \circ \widetilde{\varphi}_{2}$ implies $\left.\widetilde{\varphi}_{1}=\widetilde{\varphi}_{2}\right)$ if and only if $\tau(\widetilde{\psi})$ is a monomorphism in $\mathcal{C}$.

(c) There is a functor $K^{*}: \mathcal{C}^{\mathrm{op}} \longrightarrow \mathrm{Gps}_{*}$, defined on objects by setting

$$
K^{*}(c)=\operatorname{Ker}\left[\operatorname{Aut}_{\widetilde{\mathcal{C}}}(c) \stackrel{\tau_{c, c}}{\longrightarrow} \operatorname{Aut}_{\mathcal{C}}(c)\right]
$$

for all $c \in \mathrm{Ob}(\widetilde{\mathcal{C}})$. On morphisms, for $f \in \operatorname{Mor}_{\widetilde{\mathcal{C}}}(c, d), K^{*}(\tau(f))=[\psi]$, where $\psi \in$ $\operatorname{Hom}\left(K^{*}(d), K^{*}(c)\right)$ is the unique homomorphism such that the following square commutes in $\widetilde{\mathcal{C}}$ for all $\alpha \in K^{*}(d)$ :

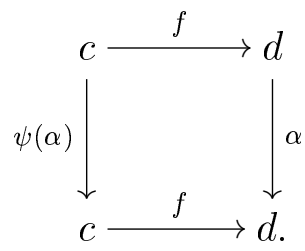

If $\tau$ is also target regular, then $K^{*}$ sends all morphisms in $\mathcal{C}$ to isomorphisms.

Proof. (a) Let $\widehat{\varphi} \in \operatorname{Mor}_{\mathcal{T}}\left(c_{0}, c_{1}\right)$ be any lifting of $\varphi$. Then there is an element $\xi \in K\left(c_{0}\right)$ such that $\widetilde{\psi \varphi}=\widetilde{\psi} \circ \widehat{\varphi} \circ \xi$, and we set $\widetilde{\varphi}=\widehat{\varphi} \circ \xi$. If $\widetilde{\varphi}^{\prime}$ is any other lifting which satisfies the same condition, then $\widetilde{\varphi}^{\prime}=\widetilde{\varphi} \circ \eta$ for some $\eta \in K\left(c_{0}\right)$, and $\eta=1$ since $\widetilde{\psi} \circ \widetilde{\varphi}=\widetilde{\psi} \circ \widetilde{\varphi}^{\prime}$ and $K\left(c_{0}\right)$ acts freely on $\operatorname{Mor}_{\mathcal{T}}\left(c_{0}, c_{2}\right)$.

(b) Fix $\widetilde{\psi} \in \operatorname{Mor}_{\widetilde{\mathcal{C}}}(c, d)$, and set $\psi=\tau_{c, d}(\widetilde{\psi})$. Assume first that $\widetilde{\psi}$ is a monomorphism, and let $\varphi_{1}, \varphi_{2} \in \operatorname{Mor}_{\mathcal{C}}\left(c_{0}, c\right)$ be such that $\psi \circ \varphi_{1}=\psi \circ \varphi_{2}$. By (a), applied to any given lifting of $\psi \circ \varphi_{1}$, there are morphisms $\widetilde{\varphi}_{1}, \widetilde{\varphi}_{2} \in \operatorname{Mor}_{\widetilde{\mathcal{C}}}\left(c_{0}, c\right)$ such that $\widetilde{\psi} \circ \widetilde{\varphi}_{1}=\widetilde{\psi} \circ \widetilde{\varphi}_{2}$ and $\tau_{c_{0}, c}\left(\widetilde{\varphi}_{i}\right)=\varphi_{i}$. Then $\widetilde{\varphi}_{1}=\widetilde{\varphi}_{2}$ since $\widetilde{\psi}$ is a monomorphism, so $\varphi_{1}=\varphi_{2}$, and $\psi$ is a monomorphism.

Conversely, assume $\psi$ is a monomorphism, and let $\widetilde{\varphi}_{1}, \widetilde{\varphi}_{2} \in \operatorname{Mor}_{\widetilde{\mathcal{C}}}\left(c_{0}, c\right)$ be such that $\widetilde{\psi} \circ \widetilde{\varphi}_{1}=\widetilde{\psi} \circ \widetilde{\varphi}_{2}$. Then $\tau_{c_{0}, c}\left(\widetilde{\varphi}_{1}\right)=\tau_{c_{0}, c}\left(\widetilde{\varphi}_{2}\right)$ since $\psi$ is a monomorphism, and hence $\widetilde{\varphi}_{1}=\widetilde{\varphi}_{2}$ by the uniqueness of the lifting in (a). So $\widetilde{\psi}$ is a monomorphism.

(c) By (a), for each $f \in \operatorname{Mor}_{\widetilde{\mathcal{C}}}(c, d)$ and each $\alpha \in K^{*}(d)$, there is a unique $\psi(\alpha) \in K^{*}(c)$ which makes square (1) commute. By juxtaposing such squares, one sees that the resulting function $\psi: K^{*}(d) \longrightarrow K^{*}(c)$ is a homomorphism which depends only on $f$, and that this defines a functor $\widetilde{K}$ from $\widetilde{\mathcal{C}}$ to $\mathrm{Gps}$. For each $c \in \operatorname{Ob}(\mathcal{C}), \widetilde{K}$ sends each morphism in $K^{*}(c)$ to an inner automorphism in $K^{*}(c)$ (by (1) again), and hence $\widetilde{K}$ factors through a functor $K^{*}$ from $\mathcal{C}$ to $\mathrm{Gps}_{*}$. 
If $\tau$ is also target regular, then each $\psi(\alpha)$ determines a unique $\alpha$ in the situation of (1), and so $K^{*}$ sends each morphism to an isomorphism.

The following lemma is proven in exactly the same way as Lemma A.7.

Lemma A.8. The following hold for any target regular functor $\tau: \widetilde{\mathcal{C}} \longrightarrow \mathcal{C}$.

(a) For any sequence $c_{0} \stackrel{\varphi}{\rightarrow} c_{1} \stackrel{\psi}{\rightarrow} c_{2}$ of morphisms in $\mathcal{C}$, and any pair of morphisms $\widetilde{\varphi} \in \operatorname{Mor}_{\widetilde{\mathcal{C}}}\left(c_{0}, c_{1}\right)$ and $\widetilde{\psi \varphi} \in \operatorname{Mor}_{\widetilde{\mathcal{C}}}\left(c_{0}, c_{2}\right)$ such that $\tau(\widetilde{\varphi})=\varphi$ and $\tau(\widetilde{\psi \varphi})=\psi \varphi$, there is a unique morphism $\widetilde{\psi} \in \operatorname{Mor}_{\widetilde{\mathcal{C}}}\left(c_{1}, c_{2}\right)$ such that $\tau(\widetilde{\psi})=\psi$ and $\widetilde{\psi} \circ \widetilde{\varphi}=\widetilde{\psi \varphi}$.

(b) For each $\widetilde{\psi} \in \operatorname{Mor}(\widetilde{\mathcal{C}}), \widetilde{\psi}$ is an epimorphism in $\widetilde{\mathcal{C}}$ in the categorical sense (i.e., $\widetilde{\varphi}_{1} \circ \widetilde{\psi}=\widetilde{\varphi}_{2} \circ \widetilde{\psi}$ implies $\left.\widetilde{\varphi}_{1}=\widetilde{\varphi}_{2}\right)$ if and only if $\tau(\widetilde{\psi})$ is an epimorphism in $\mathcal{C}$.

(c) There is a functor $K_{*}: \mathcal{C} \longrightarrow \mathrm{Gps}_{*}$, defined on objects by setting

$$
K_{*}(c)=\operatorname{Ker}\left[\operatorname{Aut}_{\widetilde{\mathcal{C}}}(c) \stackrel{\tau_{c, c}}{\longrightarrow} \operatorname{Aut}_{\mathcal{C}}(c)\right]
$$

for all $c \in \mathrm{Ob}(\widetilde{\mathcal{C}})$. On morphisms, for $f \in \operatorname{Mor}_{\widetilde{\mathcal{C}}}(c, d), K_{*}(\tau(f))=[\psi]$, where $\psi \in$ $\operatorname{Hom}\left(K_{*}(c), K_{*}(d)\right)$ is the unique homomorphism such that the following square commutes in $\widetilde{\mathcal{C}}$ for all $\alpha \in K_{*}(c)$ :

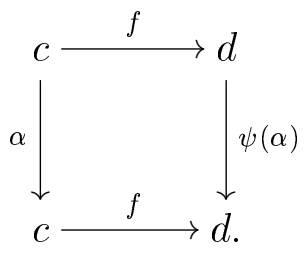

The obstruction theory for describing source or target regular extensions of $\mathcal{C}$ by a given functor $K$ from $\mathcal{C}$ to $\mathrm{Gps}_{*}$ has been studied by Hoff [Hf]. For simplicity here, we handle only the case where $K$ takes values in abelian groups. The nonabelian case is handled in [Hf] by using higher limits of functors with values in nonabelian groups (when they exist). Alternatively, if one assumes that $K$ restricts to a functor $Z K$ (where $Z K(c)=Z(K(c))$ ), then there is an obstruction theory which involves the groups $\lim ^{i}(Z(K))$ for $i=2,3$.

Now fix $K: \mathcal{C}^{\text {op }} \longrightarrow \mathrm{Ab}$, and let $\widetilde{\mathcal{C}} \stackrel{\tau}{\longrightarrow} \mathcal{C}$ be a source regular extension of $\mathcal{C}$ by $K$. Choose a section

$$
s: \operatorname{Mor}(\mathcal{C}) \longrightarrow \operatorname{Mor}(\widetilde{\mathcal{C}}) .
$$

For each pair of composable morphisms $c_{0} \stackrel{\varphi}{\longrightarrow} c_{1} \stackrel{\psi}{\longrightarrow} c_{2}$ in $\mathcal{C}$, let $\omega(\varphi, \psi) \in K\left(c_{0}\right)$ be the unique element such that

$$
s(\psi) \circ s(\varphi)=s(\psi \varphi) \circ \omega(\varphi, \psi) \in \operatorname{Mor}_{\widetilde{\mathcal{C}}}\left(c_{0}, c_{2}\right) .
$$

This defines an element $\omega \in \bar{C}^{2}(\mathcal{C} ; K)$ (see Lemma A.1).

We next check that $d \omega=0$. Fix a triple $c_{0} \stackrel{\varphi}{\longrightarrow} c_{1} \stackrel{\psi}{\longrightarrow} c_{2} \stackrel{\chi}{\longrightarrow} c_{3}$ of composable morphisms. Associativity in $\widetilde{\mathcal{C}}$ gives the relation

$$
\begin{aligned}
& s(\chi) \circ(s(\psi) \circ s(\varphi))=s(\chi \psi \varphi) \circ \omega(\psi \varphi, \chi) \circ \omega(\varphi, \psi) \\
= & (s(\chi) \circ s(\psi)) \circ s(\varphi)=s(\chi \psi \varphi) \circ \omega(\varphi, \chi \psi) \circ K(\varphi)(\omega(\psi, \chi)) .
\end{aligned}
$$

This gives the relation

$$
\omega(\psi \varphi, \chi) \circ \omega(\varphi, \psi)=\omega(\varphi, \chi \psi) \circ K(\varphi)(\omega(\psi, \chi)) \in K\left(c_{0}\right) .
$$


This proves that $d \omega=0$, and hence that $\omega \in \bar{Z}^{2}(\mathcal{C} ; K)$.

It remains to show that the class $[\omega] \in \lim ^{2}(K)$ is independent of the choice of section $s$. Let $s^{\prime}$ be another section, and let $\beta \in \bar{C}^{1}(\mathcal{C} ; K)$ be such that $s^{\prime}(\varphi)=s(\varphi) \circ \beta(\varphi)$ for all $\varphi \in \operatorname{Mor}_{\mathcal{C}}\left(c_{0}, c_{1}\right)$. Let $\omega^{\prime} \in \bar{C}^{2}(\mathcal{C} ; K)$ be the cochain analogous to the cochain $\omega$ already defined. Then for $\varphi$ and $\psi$ as above,

$$
\begin{aligned}
s^{\prime}(\psi) \circ s^{\prime}(\varphi) & =s^{\prime}(\psi \varphi) \circ \omega^{\prime}(\varphi, \psi)=s(\psi \varphi) \circ \beta(\psi \varphi) \circ \omega^{\prime}(\varphi, \psi) \\
& =s(\psi) \circ \beta(\psi) \circ s(\varphi) \circ \beta(\varphi)=s(\psi) \circ s(\varphi) \circ K(\varphi)(\beta(\psi)) \circ \beta(\varphi) .
\end{aligned}
$$

Thus, in the abelian group $K\left(c_{0}\right)$,

$$
\omega(\varphi, \psi)^{-1} \circ \omega^{\prime}(\varphi, \psi)=\beta(\psi \varphi)^{-1} \circ K(\varphi)(\beta(\psi)) \circ \beta(\varphi)=d \beta(\varphi, \psi) ;
$$

and this proves that $\omega^{\prime}=\omega \cdot d \beta$.

We have now shown how to assign a unique element of $\lim ^{2}(K)$ to each extension of $\mathcal{C}$ by $K$. It is easy to show, using similar manipulations, that all elements of $\lim ^{2}(K)$ can be realized in this way, and that extensions corresponding to the same element of $\lim ^{2}(K)$ are isomorphic. This can all be summarized in the following proposition:

Proposition A.9. Fix a small category $\mathcal{C}$, and a functor $K: \mathcal{C}^{\mathrm{op}} \longrightarrow \mathrm{Ab}$. Then there is a bijective correspondence between isomorphism classes of source regular extensions of $\mathcal{C}$ by $K$ and the group $\underset{\mathcal{C}}{\lim ^{2}}(K)$.

We next look at geometric realizations of source and target regular functors. The following, very general result can be used in many situations to prove that certain maps between linking or transporter systems are fibrations with fiber $B A$, for a certain $p$-group $A$.

Proposition A.10. Fix a group $G$, and a functor $\tau: \widetilde{\mathcal{C}} \longrightarrow \mathcal{C}$ between small categories which is both source regular and target regular. Assume $|\mathcal{C}|$ is connected, fix $c_{0} \in \mathrm{Ob}(\widetilde{\mathcal{C}})$, and set $G=\operatorname{Ker}\left(\tau_{c_{0}, c_{0}}\right)$. Then the $\operatorname{map}|\widetilde{\mathcal{C}}| \stackrel{|\tau|}{\longrightarrow}|\mathcal{C}|$ of geometric realizations is a fiber bundle with fiber $B G$.

Proof. We prove this by constructing a topological group, together with a principal bundle over that group of which $|\tau|$ is an associated bundle. For each $c \in \operatorname{Ob}(\mathcal{C})=$ $\operatorname{Ob}(\widetilde{\mathcal{C}})$, set $K(c)=\operatorname{Ker}\left(\tau_{c, c}\right) \triangleleft \operatorname{Aut}_{\widetilde{\mathcal{C}}}(c)$.

Let $\mathcal{G}$ be the category of self equivalences of $\mathcal{B}(G)$, where morphisms in $\mathcal{G}$ are natural isomorphisms of functors. Thus

$$
\operatorname{Ob}(\mathcal{G})=\operatorname{Aut}(G) \quad \text { and } \quad \operatorname{Mor}_{\mathcal{G}}(\alpha, \beta)=\left\{g \in G \mid \beta=c_{g} \circ \alpha\right\} .
$$

Let $\mathcal{P}$ be the category of functors $\mathcal{B}(G) \longrightarrow \widetilde{\mathcal{C}}$ which go isomorphically to one of the "fibers" of $\tau$; more precisely:

$$
\begin{aligned}
\operatorname{Ob}(\mathcal{P}) & =\{(c, \lambda) \mid c \in \mathrm{Ob}(\mathcal{C}), \lambda \in \operatorname{Iso}(G, K(c))\} \\
\operatorname{Mor}_{\mathcal{P}}((c, \lambda),(d, \mu)) & =\left\{\varphi \in \operatorname{Mor}_{\widetilde{\mathcal{C}}}(c, d) \mid \mu=c_{\varphi} \circ \lambda\right\} .
\end{aligned}
$$

Consider the following square of categories and functors:

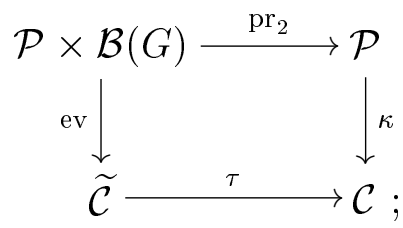


where $\kappa$ is the forgetful functor $\kappa(c, \lambda)=c$, and ev is the "evaluation functor"

$$
\mathrm{ev}(g,(c, \lambda) \stackrel{\varphi}{\rightarrow}(d, \mu))=(c \underset{=\mu(g) \circ \varphi}{\stackrel{\varphi \circ \lambda(g)}{\longrightarrow}} d) .
$$

For each $n \geq 0$, let $\mathcal{G}_{n}$ denote the set of $n$-simplices in the nerve of $\mathcal{G}$, and similarly for the other categories. Then $\mathcal{G}_{n}$ is a group with multiplication

$$
\left(\alpha_{0} \stackrel{g_{1}}{\longrightarrow} \alpha_{1} \stackrel{g_{2}}{\longrightarrow} \cdots\right) \cdot\left(\beta_{0} \stackrel{h_{1}}{\longrightarrow} \beta_{1} \stackrel{h_{2}}{\longrightarrow} \cdots\right)=\left(\alpha_{0} \circ \beta_{0} \stackrel{g_{1} \cdot \alpha_{0}\left(h_{1}\right)}{\longrightarrow} \alpha_{1} \circ \beta_{1} \stackrel{g_{2} \cdot \alpha_{1}\left(h_{2}\right)}{\longrightarrow} \cdots\right),
$$

and the face and degeneracy maps are all homorphisms. So $|\mathcal{G}|$ is a topological group. Furthermore, $\mathcal{G}_{n}$ has a free right action on $\mathcal{P}_{n}$ defined by

$$
\left(\left(c_{0}, \lambda_{0}\right) \stackrel{\varphi}{\rightarrow}\left(c_{1}, \lambda_{1}\right)\right) \circ\left(\alpha_{0} \stackrel{g}{\rightarrow} \alpha_{1}\right)=\left(\left(c_{0}, \lambda_{0} \circ \alpha_{0}\right) \stackrel{\varphi \circ \lambda_{0}(g)}{\longrightarrow}\left(c_{1}, \lambda_{1} \circ \alpha_{1}\right)\right) ;
$$

and a left action on $\mathcal{B}(G)_{n}$ defined by

$$
\left(\alpha_{0} \stackrel{g}{\rightarrow} \alpha_{1}\right) \cdot(* \stackrel{h}{\rightarrow} *)=\left(* \stackrel{g \cdot \alpha_{0}(h)}{\longrightarrow} *\right) ;
$$

actions which again commute with face and degeneracy maps. Together with the functors $\kappa$ and ev, these actions induce bijections

$$
\mathcal{P}_{n} / \mathcal{G}_{n} \cong \mathcal{C}_{n} \quad \text { and } \quad \mathcal{P}_{n} \times_{\mathcal{G}_{n}} \mathcal{B}(G)_{n} \cong \widetilde{\mathcal{C}_{n}}
$$

for all $n$. Hence the geometric realizations $|\mathrm{ev}|$ and $|\kappa|$ are principal $|\mathcal{G}|$-fibrations (see, e.g., [May, §§18-20] or [GJ, Corollary V.2.7]). In particular, ev induces a homeomorphism $|\widetilde{\mathcal{C}}| \cong|\mathcal{P}| \times_{|\mathcal{G}|} B G$, and hence $|\tau|$ is a fiber bundle with fiber $B G$ associated to the principal bundle $|\mathcal{P}| \stackrel{|\kappa|}{\longrightarrow}|\mathcal{C}|$.

We now finish by describing how the Grothedieck spectral sequence applies to describe higher limits over a target regular extension.

Proposition A.11. Fix small categories $\widetilde{\mathcal{C}}$ and $\mathcal{C}$, and let $\tau: \widetilde{\mathcal{C}} \longrightarrow \mathcal{C}$ be a target regular functor. For each $c \in \mathrm{Ob}(\mathcal{C})$, set

$$
K(c)=\operatorname{Ker}\left[\operatorname{Aut}_{\widetilde{\mathcal{C}}}(c) \stackrel{\tau}{\longrightarrow} \operatorname{Aut}_{\mathcal{C}}(c)\right]
$$

Let $\Phi: \widetilde{\mathcal{C}}^{\mathrm{op}} \longrightarrow \mathrm{Ab}$ be any functor. Then there is a spectral sequence

$$
E_{2}^{i j}={\underset{\mathcal{C}}{\lim }}^{i}\left(H^{j}(K(-) ; \Phi(-))\right) \Longrightarrow{\underset{\widetilde{\mathcal{C}}}{\lim }}^{i+j}(\Phi) \text {. }
$$

Proof. This is shown using the Grothendieck spectral sequence (cf. [Wb, §5.8]). Consider the following triangle of categories and functors:

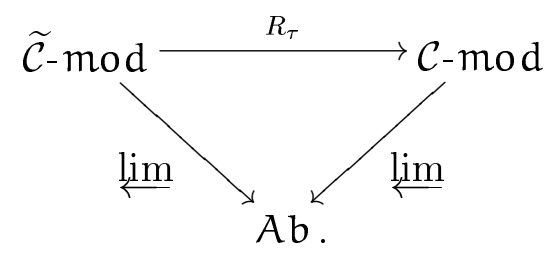

Here, $R_{\tau}: \widetilde{\mathcal{C}}-\bmod \longrightarrow \mathcal{C}$-mod is right Kan extension by $\tau$. Since $R_{\tau}$ is right adjoint to an exact functor (composition with $\tau^{\mathrm{op}}$ ), it sends injectives to injectives.

Let $R_{\tau}^{i}$ be the $i$-th derived functor of $R_{\tau}$. Thus for all $\Phi$ in $\widetilde{\mathcal{C}}$-mod and all $c \in \operatorname{Ob}(\mathcal{C})$,

$$
R_{\tau}^{j}(\Phi)(c)=\underset{(\tau \downarrow c)^{\mathrm{op}}}{\lim ^{j}}\left(\Phi \circ \kappa_{c}^{\mathrm{op}}\right)
$$


where $\kappa_{c}: \tau \downarrow c \longrightarrow \widetilde{\mathcal{C}}$ is the forgetful functor. The Grothendieck spectral sequence for the triangle (4) takes the form

$$
E_{2}^{i j}={\underset{\mathcal{C}}{\lim }}^{i}\left(R_{\tau}^{j}(\Phi)\right) \Longrightarrow{\underset{\widetilde{\mathcal{C}}}{\lim }}^{i+j}(\Phi) .
$$

Now, $\tau \downarrow c$ is the overcategory with objects the pairs $(d, \varphi)$ for $d \in \operatorname{Ob}(\widetilde{\mathcal{C}})$ and $\varphi \in$ $\operatorname{Mor}_{\mathcal{C}}(\tau(d), c)$, and where

$$
\operatorname{Mor}_{\tau \downarrow c}\left((d, \varphi),\left(d^{\prime}, \psi\right)\right)=\left\{f \in \operatorname{Mor}_{\widetilde{\mathcal{C}}}\left(d, d^{\prime}\right) \mid \psi \circ \tau(f)=\varphi\right\} .
$$

Consider the functors

$$
\mathcal{B}(K(c)) \stackrel{S}{\longrightarrow} \tau \downarrow c \stackrel{T}{\longrightarrow} \mathcal{B}(K(c)),
$$

where $S$ sends the unique object of $\mathcal{B}(K(c))$ to $(c, \mathrm{Id})$ and sends $\alpha \in K(c)$ to $\alpha \in$ $\operatorname{Aut}_{\tau \downarrow c}(c, \mathrm{Id})$; and where $T$ is defined as follows. Fix an arbitrary map of sets

$$
s: \operatorname{Ob}(\tau \downarrow c) \longrightarrow \operatorname{Mor}(\widetilde{\mathcal{C}})
$$

such that for all $(d, \varphi), s(d, \varphi) \in \operatorname{Mor}_{\widetilde{\mathcal{C}}}(d, c)$ and $\tau_{d, c}(s(d, \varphi))=\varphi$. For each morphism $f$ in $\tau \downarrow c$ from $(d, \varphi)$ to $\left(d^{\prime}, \psi\right)$, there is (since $\tau$ is target regular) a unique element $T(f) \in K(c)$ such that

$$
T(f) \circ s(d, \varphi)=s\left(d^{\prime}, \psi\right) \circ f .
$$

This defines $T$. Furthermore, $T \circ S=\operatorname{Id}_{\mathcal{B}(K(c))}$, while $s$ defines a natural transformation of functors from $S \circ T$ to $\operatorname{Id}_{\tau \downarrow c}$.

It now follows that for all $c$ and $j$,

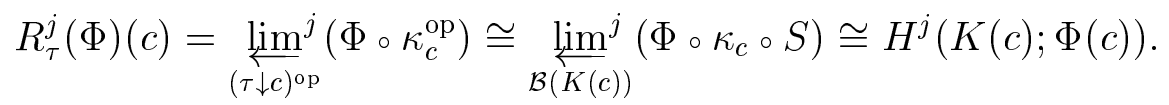

The spectral sequence (3) now follows from this together with (5).

\section{REFERENCES}

[BK] P. Bousfield \& D. Kan, Homotopy limits, completions, and localizations, Lecture notes in math. 304, Springer-Verlag (1972)

[BCGLO1] C. Broto, N. Castellana, J. Grodal, R. Levi, \& B. Oliver, Subgroup families controlling $p$-local finite groups, Proc. London Math. Soc. (to appear)

[BCGLO2] C. Broto, N. Castellana, J. Grodal, R. Levi, \& B. Oliver, Extensions of p-local finite groups, Trans. Amer. Math. Soc. (to appear)

[BLO1] C. Broto, R. Levi, \& B. Oliver, Homotopy equivalences of $p$-completed classifying spaces of finite groups, Invent. math. 151 (2003), 611-664

[BLO2] C. Broto, R. Levi, \& B. Oliver, The homotopy theory of fusion systems, Journal Amer. Math. Soc. 16 (2003), 779-856

[BLO3] C. Broto, R. Levi, \& B. Oliver, Discrete models for the $p$-local homotopy theory of compact Lie groups, in preparation

[GZ] P. Gabriel \& M. Zisman, Calculus of fractions and homotopy theory, Springer-Verlag (1967)

[GJ] P. Goerss \& R. Jardine, Simplicial homotopy theory, Birkhäuser (1999)

[Go] D. Gorenstein, Finite groups, Harper \& Row (1968)

[Hf] G. Hoff, Cohomologies et extensions de categories, Math. Scand. 74 (1994), 191-207.

[JMO] S. Jackowski, J. McClure, \& B. Oliver, Homotopy classification of self-maps of $B G$ via $G$ actions, Annals of Math. 135 (1992), 184-270

[May] P. May, Simplicial objects in algebraic topology, Van Nostrand (1967)

[O1] B. Oliver, Higher limits via Steinberg representations, Comm. in Algebra 22 (1994), 1381-1393

[O2] B. Oliver, Equivalences of classifying spaces completed at odd primes, Math. Proc. Camb. Phil. Soc. 137 (2004), 321-347 
[Pg] Ll. Puig, Unpublished notes (ca. 1990)

[Q] D. Quillen, Algebraic $K$-theory I, Lecture notes in mathematics 341 (1973), 77-139

[Rz] A. Ruiz, Exotic subsystems of finite index in the fusion systems of general linear groups over finite fields, preprint

[St] R. Stancu, Equivalent definitions of fusion systems, preprint

[Wb] C. Weibel, An introduction to homological algebra, Cambridge Univ. Press (1994)

LaGa, Institut Galilée, Av. J-B Clément, 93430 Villetaneuse, France

E-mail address: bobol@math.univ-paris13.fr

Departamento de Matemática, Instituto Superior Técnico, Av. Rovisco Pais, 1049001 Lisboa, Portugal

E-mail address: jventura@math.ist.utl.pt 